\title{
Taxonomic catalogue of the family Ochteridae with description of Ochterus papaceki sp. nov. from Socotra Island and Tanzania (Hemiptera: Heteroptera)
}

\author{
Petr KMENT ${ }^{1)}$, Attilio CARAPEZZA ${ }^{2)}$ \& Zdeněk JINDRA ${ }^{3)}$ \\ 1) Department of Entomology, National Museum, Cirkusová 1740, CZ-19300 Praha 9- Horní Počernice, Czech Republic; e-mail: sigara@post.cz \\ ${ }^{2)}$ University of Palermo; corresponding address: via Sandro Botticelli 15, 90144 Palermo, Italy; e-mail: attilio.carapezza@unipa.it \\ ${ }^{3)}$ Department of Plant Protection, Faculty of Agrobiology, Food and Natural Resources, Czech University of Agriculture, CZ-165 21 \\ Praha 6 - Suchdol, Czech Republic; e-mail: palomena@seznam.cz
}

Accepted:

$5^{\text {th }}$ February 2020

Published online: $29^{\text {th }}$ February 2020

\begin{abstract}
The world catalogue of the family Ochteridae (Hemiptera: Heteroptera: Nepomorpha: Ochteroidea) is compiled. The fossil subfamily Propreocorinae Popov, Dolling \& Whalley, 1994, recently excluded from Ochteridae by other authors, is formally raised to family rank as Propreocoridae stat. nov. The fossil genus Meropachys Popov, 1986 is found to be a junior homonym of Meropachys Burmeister, 1835 (Hemiptera: Heteroptera: Coreidae) and is replaced by a new substitute name, Yuripachys nom. nov., resulting in one new combination: Yuripachys dubius (Popov, 1986) comb. nov. Neochterus Mahner, 1993 (originally proposed as subgenus of Ochterus Latreille, 1807) is considered an unavailable name. Ochterus papaceki Kment \& Carapezza sp. nov. is described and illustrated as a new species from the island of Socotra (Yemen) and Tanzania. Gender agreement in Ochterus paucistriata Baehr, 1990 is corrected to O. paucistriatus Baehr, 1990. The name Ochterus perbosci (Guérin-Méneville, 1843), an incorrect subsequent spelling of $O$. perboscii in prevailing usage, is fixed according to Article 33.3.1 of the ICZN (1999). The spelling of Angulochterus quadrimaculatus Yao, Zhang \& Ren, 2011 is corrected according to Article 32.5.1 of ICZN (1999). The original publication of Ochterus barberi Schell, 1943, O. bidentatus Schell, 1943, O. hungerfordi Schell, 1943, and O. parvus Schell, 1943 is clarified. The name bearing types of Ochterus barberi, O. bidentatus, O. hungerfordi, O. parvus, O. perbosci, and Pelogonus splendidulus Montandon, 1898 (= Ocyochterus victor (Bolívar, 1879)) are considered as lectotypes. The family Ochteridae currently includes 3 recent genera and 88 described species group taxa (84 species and 4 subspecies): Megochterus Jaczewski, 1934 (2 species from Australia), Ochterus ( 80 species and 4 subspecies distributed worldwide), and Ocyochterus Drake \& Gómez-Menor, 1954 (2 species from NW South America). The fossil record of Ochteridae currently includes 4 genera and 5 species: Angulochterus Yao, Zhang \& Ren, 2011 (1 species from Early Cretaceous of China), Floricaudus Yao, Ren \& Shih, 2011 (1 species from Early Cretaceous of China), Pristinochterus Yao, Cai \& Ren, 2007 (2 species from Late Jurassic to Early Cretaceous of China), and Riegerochterus Popov \& Heiss, 2014 (1 species from Miocene Dominican amber). In addition the fossil genus Yuripachys (1 species from Early Creatceous of Mongolia) is classified as Ochteridae incertae sedis, Propreocoris Popov, Dolling \& Whalley, 1994 (1 species from Early Jurassic of England) as common ancestor of Ochteridae and Gelastocoridae in its own family Propreocoridae, and Grimaldinia Popov \& Heiss, 2014 (1 species from Burmese Amber) and Heterochterus Evans, 1971 (1 species based on an isolated wing, Late Triassic of Australia) as Ochteroidea incertae sedis. The following new records are provided: Ochterus aeneifrons surinamensis Nieser, 1975 (Colombia), O. caffer (Stål, 1855) (Mozambique, Sudan), O. feae (Laos, Thailand), O. marginatus marginatus (Latreille, 1804) (China: Anhui, Shaanxi; Central African Republic; India: Rajasthan; Oman; Sudan), and $O$. nicobarensis Chandra \& Jehamalar, 2012 (Myanmar). The distribution of all species is reviewed and the zoogeographic patterns and biodiversity of Ochteridae are discussed.
\end{abstract}

Key words. Heteroptera, Nepomorpha, Ochteroidea, Ochteridae, Propreocorinae, new species, new substitute name, catalogue, nomenclature, taxonomy, fossils, distribution 


\section{Introduction}

Velvety shore bugs of the family Ochteridae represent a small monophyletic lineage of aquatic bugs of the infraorder Nepomorpha. Ochteridae, the toad bugs (Gelastocoridae), and two fossil families, Propreocoridae stat. nov. (Popov et al. 1994) and Pseudonerthridae (López Ruf et al. 2005) jointly form the monophyletic Ochteroidea (MAHNER 1993; HebsgaARD et al. 2004; Yao et al. 2011; Li et al. 2012, 2014; WANG et al. 2016; WEIRAUCH et al. 2019; YE et al. 2020). WANG et al. (2016) dated the split between Ochteridae and Gelastocoridae to the Early Jurassic based on a molecular phylogenetic analysis calibrated by fossils. This estimate is in agreement with the fossil record, which includes well preserved compressions from the Late Jurassic and the Early Cretaceous of China, which are morphologically similar to modern Ochteridae (YAO et al. 2007, 2011). The fossil Propreocoridae, considered a stem group of Ochteroidea (GRIMALDI \& ENGEL 2005, YAO et al. 2011) comes from Early Jurassic of England, and a single hind wing described as Heterochterus timmsii Evans, 1971, tentatively assigned to Ochteroidea, was found in the Late Triassic of Queensland (Australia) (Evans 1971, ShCHERBAKOv \& Popov 2002).

The Ochteroidea is a peculiar group of insects, being the only lineage of the secondarily aquatic Nepomorpha which returned back to a terrestrial way of life. The recent Ochteridae may be found mostly in riparian habitats, such as margins of streams or ponds, typically located on sandy and stony places with little vegetation, which are usually sunlit (although some species seem to prefer rather shaded habitats). Their cryptic coloration makes them nearly invisible when they are sitting on their preferred substrate. They can run rapidly, jump when disturbed, and they are also capable of flight (Bobb 1951, Gapud \& San Valentin 1977, Menke 1979, Boulard \& Coffin 1991, Schuh \& Slater 1995, Nieser \& Chen 1999, Andersen \& Weir 2004, Chen et al. 2005, Schun \& Weirauch 2020). Recently, Polhemus \& Polhemus (2016) reported Ochterus explanatus Polhemus \& Polhemus, 2016 and O. obscurus Polhemus \& Polhemus, 2016 from leaf litter samples collected in high elevation tropical montane and cloud forests in Central America. Despite the close phylogenetic relationship, Ochteridae and Gelastocoridae are not similar to each other. While Gelastocoridae are generally larger and more robust, equipped with raptorial fore legs, resembling small toads and often covered with mud, Ochteridae are smaller, gracile, widely oval, velvety black often with small yellowish spots (e.g., Schun \& Slater 1995, Andersen \& Weir 2004, Chen et al. 2005, Schun \& WeIRAuch 2020). Ochteridae are habitually much more similar to the leptopodomorphan family Saldidae, sharing with Ochteridae the oval body (though more ovoid in Saldidae), head with large kidney-shaped eyes, usually dark colouration of dorsum with whitish or yellowish spots, and life in the riparian habitats. Both these families are an interesting case of convergent evolution, though both can be easily distinguished by the shape of antennae, which are long and robust in Saldidae (as well as in the remaining terrestrial or semiaquatic Heteroptera) (e.g., Schun \& Slater 1995, Schuh \& Weirauch 2020), and very short and inconspicuous in Ochteridae. The very short antennae, usually concealed in grooves below the eyes, are an important synapomorphy for Nepomorpha, connected with their adaptation to the subaquatic way of life (e.g., Rieger 1976, Mahner 1993, Schuh \& Slater 1995, Andersen \& Weir 2004, Hebsgaard et al. 2004); convergently, shortened antennae are also known in many beetle families with aquatic adults (Coleoptera: Adephaga and Polyphaga) (e.g., Beutel \& Leschen 2016).

Due to the morphological homogeneity of Ochteridae, only three recent genera and 88 species group taxa are currently recognized (see below). The main diagnostic characters at the species level include the structure and striation of the frons (the so called frontal plate), shape and structure of pronotum, colouration of body dorsum, shape of the male genital capsule, and especially, the structure of the right paramere (e.g., ANDERSEN \& WeIR 2004; PolHEMUS $\&$ Polhemus 2012, 2016). Although a world catalogue or monograph of Ochteridae has never been compiled, there are several valuable revisions, monographs or catalogues dealing with faunas of particular zoogeographical regions: Afrotropical Region (Kormilev 1973, Polhemus \& Polnemus 2008), Palaearctic Region (Polhemus 1995a), Oriental Region (Kormilev 1971, Nieser \& CHEN 1992, Polnemus \& Polhemus 2012), Malesia (Kormilev 1971; Nieser \& Chen 1992, 1999; Chen et al. 2005; Polhemus \& Polhemus 2012, 2015), Philippines (Gapud \& SAN VALentin 1977; GAPud 1981, 1986, 2003; Chen et al. 2005), New Guinea (Kormilev 1971, Baehr 1990b, Chen et al. 2005), Australia (Kormilev 1971; BAEHR 1989, 1990a,b; Cassis \& Gross 1995; Andersen \& Weir 2004), North America (Schell 1943a,b; Polhemus \& Polhemus 1988a, 2016), Central America (Champion 1901; Schell 1943a,b; Heckman 2011; Polhemus \& Polhemus 2016), and South America (Schell 1943a,b; NieSer 1975; Heckmann 2011; Moreira et al. 2011, 2019; CiAnFERoni 2012; Cordeiro et al. 2014; Polnemus \& Polnemus 2014, 2016; Barbosa \& Rodrigues 2015). Despite the considerable progress in Ochteridae taxonomy since 1971, there are still many species awaiting description. In order to assist in future taxonomic and phylogenetic efforts in Ochteridae, we provide here a taxonomic catalogue of all the described taxa of this family, extant as well as fossil, we review their distribution and discuss their zoogeographic patterns.

\section{Material and methods}

The presented description of Ochterus papaceki Kment \& Carapezza sp. nov. is based on material collected mostly in Socotra by Attilio Carapezza and by members of the Czech expeditions in 2010 and 2012. For further information on results of the Czech expeditions see e.g. HÁJEK \& BEZDĚK (2012, 2014, 2017, 2019), BEZDĚK \& HÁJEK (2017), KMENT et al. (2017) or Biondi et al. (2019). For brief description of the natural conditions of Socotra see BATELKA (2012). For details on the Iranian localities sampled by the Czechoslovak-Iranian expeditions in 1970, 1973 and 1977 see Hoberlandt $(1974,1981,1983)$.

In quoting the labels of the holotype, a slash (/) is used to divide data on different rows of one label, double slash (//) 
is used to divide data on different labels, authors' comments are given in square brackets [ ], and the abbreviation ' $p$ ' is used to indicate printed text. Localities of paratypes and other specimens are standardized. The names of the Socotran localities follow BEZDĚK et al. (2012).

The specimens examined are deposited in the following collections:

ACPI Attilio Carapezza collection, Palermo, Italy;

AMNH American Museum of Natural History, New York, USA;

AMSA Australian Museum, Sydney, Australia;

BMKB Brunei Museum, Kota Baru, Brunei;

BMNH The Natural History Museum, London, United Kingdom;

BPBM Bernice P. Bishop Museum, Honolulu, Hawaii, USA;

CASC California Academy of Sciences, San Francisco, California, USA;

CNUB Key Laboratory of Insect Evolution and Environmental Changes, College of Life Sciences, Capital Normal University, Beijing, China;

CRNG Christian Rieger collection, Nürtingen, Germany;

CZMA Coleção Zoológica do Maranhão, Centro de Estudos Superiores de Caxias, Caxias, Maranhão, Brazil;

EHIA Ernst Heiss collection, Innsbruck, Austria;

EIHU Entomological Institute, Hokkaido University, Sapporo, Japan; IEEM Instituto Español de Entomología, Madrid, Spain;

ISNB Institut Royal des Sciences Naturelles de Belgique, Bruxelles, Belgium;

IZAS Institute of Zoology, Academia Sinica, Beijing, China;

JDKD Jakob Damgaard collection, Zoological Museum, Kobenhavn, Denmark;

JTPC John T. Polhemus collection, Englewood, Colorado, USA;

MACN Museo Argentino de Ciencias Naturales "Bernardino Rivadavia", Buenos Aires, Argentina;

MCSN Museo Civico di Storia Naturale 'Giacomo Doria', Genoa, Italy;

MMBC Moravian Museum, Brno, Czech Republic;

MNCN Museo Nacional de Ciencias Naturales, Madrid, Spain;

MNHN Muséum National d'Histoire Naturelle, Paris, France;

MUDH Museon, The Hague, the Netherlands;

MZFN Museo Zoologico, Università degli Studi di Napoli Federico II (former Istituto di Zoologia, Universita di Napoli), Napoli, Italy;

MZLU Museum of Zoology, Lund University, Lund, Sweden;

NCTN Nico Nieser collection, Tiel, the Netherlands;

NHMW Naturhistorisches Museum in Wien, Vienna, Austria;

NHRS Naturhistoriska Riksmuseet, Stockholm, Sweden;

NMPC National Museum, Prague, Czech Republic;

NZSI National Zoological Collection, Zoological Survey of India, Calcutta, India;

PIN Paleontological Institute, Russian Academy of Sciences, Moscow, Russia;

RMNH Naturalis Biodiversity Center (former Rijksmuseum van Natuurlijke Historie, Leiden, The Netherlands;

QMBA Queensland Museum, Brisbane, Australia;

SEMC Snow Entomological Museum, University of Kansas, Lawrence, Kansas, USA;

SMNS Staatliches Museum für Naturkunde, Stuttgart, Germany;

UM Zoölogisch Museum der Rijksuniversiteit, Utrecht, The Netherlands;

UPLB Museum of Natural History, University of the Philippines, Los Baños College, Laguna, Philippines;

USNM National Museum of Natural History, Smithsonian Institution, Washington DC, USA;

VPGP Victor P. Gapud collection, Department of Entomology, University of the Philippines, Los Baños College, Laguna, Philippines;

WAMA Western Australian Museum, Perth, Australia;

ZJPC Zdeněk Jindra collection, Prague, Czech Republic;

ZMAN Zoölogisch Museum, University of Amsterdam, Amsterdam, The Netherlands;
ZMAS Zoological Institute, Russian Academy of Sciences, St. Petersburg, Russia;

ZMPA Zoological Institute, Polish Academy of Sciences, Warsaw, Poland;

ZMUH Zoologisches Museum, Universität von Hamburg, Hamburg, Germany;

ZRCS Zoological Reference Collection, Lee Kong Chian Natural History Museum (former Raffles Museum of Biodiversity Research), Singapore;

ZSMC Zoologische Staatssammlung, München, Germany.

Measurements were taken using a Leica MZ75 stereomicroscopes provided with an ocular micrometer and subsequently standardized to absolute values. Non-coated specimens were examined by a Hitachi S-3700N environmental scanning electron microscope at the Department of Palaeontology, National Museum, Praha. Habitus photographs were taken using a Canon MP-E $65 \mathrm{~mm}$ macro lens attached to a Canon EOS 550D camera, light microscope photographs were taken using an Olympus BX44 microscope with an attached Canon EOS 1100D camera. The photographs were stacked from multiple layers using the Helicon Focus 5.1 Pro software.

The general morphological terminology follows mostly AndERSEN \& Weir (2004), TsAi et al. (2011), KMENT et al. (2016, 2019) and RÉDei (2017); male genitalia (PolHemus \& Polhemus 2016), and antennomeres ZrZavÝ (1990).

\section{Description of a new species}

Ochterus papaceki Kment \& Carapezza, sp. nov. (Figs 1-6, 9-12)

Type locality. Yemen, Socotra, Noged plain, lower part of Wadi Matyaf, $12^{\circ} 27^{\prime} 13.50^{\prime \prime} \mathrm{N} 54^{\circ} 18^{\prime} 13.95^{\prime \prime} \mathrm{E}$.

Type material. HoLotyPe: $\delta$ (Fig. 1), 'SOCOTRA 21.I.2014 / Noged - lower part / of Wadi Matyaf / leg. A. Carapezza // COLLECTIO / NATIONAL MUSEUM / Praha, Czech Republic // $\lesssim$ [p] // HOLOTYPUS / OCHTERUS / PAPACEKI / sp. nov. / det. Kment \& Carapezza 2020 [p, red label]' (NMPC). The holotype is card-mounted, complete, with dissected genital capsule and right paramere glued on the same piece of card. PARATYPES (16 12 ㅇ 1 L5): YEMEN: SoCOTRA: Hallah Arhar [= Halla area, Arher] (spring), $15 \mathrm{~m}, 12^{\circ} 33.0^{\prime} \mathrm{N} 54^{\circ} 27.6^{\prime} \mathrm{E}, 11 . x i .2010,2$

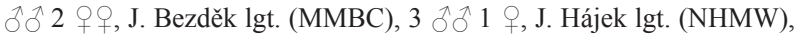
1 ㅇ, P. Hlaváč lgt. (MMBC); Halla area, Arher $\left(12^{\circ} 33.0^{\prime} \mathrm{N} 54^{\circ} 27.6^{\prime} \mathrm{E}\right)$, freshwater spring in sand dune, $5 \mathrm{~m}$ a.s.1., 9.-10.vi.2012, $4 \hat{\delta} 4$ 우 1 L5, Socotra expedition 2012: J. Bezděk, J. Hájek, V. Hula, P. Kment, I. Malenovský, J. Niedobová \& L. Purchart lgt. (3 đđ 3 q 91 L5 NMPC, 1 of 1 BMNH); Noged, lower part of wadi Matyaf, $12^{\circ} 27^{\prime} 13.50^{\prime \prime} \mathrm{N}$

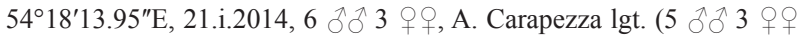
ACPI, $1 \precsim$ NMPC). TANZANIA: NE, $100 \mathrm{~km} \mathrm{SW}$ of Tanga, Makinda env., 14.iii.2002, 1 † 1 , M. Snížek lgt. (ZJPC). All the paratypes bear the following label: 'PARATYPUS / OCHTERUS / PAPACEKI/ sp. nov. / det. Kment \& Carapezza 2020 [p, red label]’.

Description. Adult. Colouration (Fig. 1). Body dorsally very dark brown to black with following yellowish pattern: flattened lateral pronotal margins; posterior pronotal margin narrowly, with larger spots on posterolateral angles and medially; costal margin of corium narrowly (anteriorly somewhat wider than posteriorly), connected with two round lateral spots, one ca. in middle and second in posterior half of embolium; one nebulous spot (sometimes indistinct) posteriad of $\mathrm{R}+\mathrm{M}$ vein; two nebulous spots in anterodistal and posterodistal angles of corium (adjacent to membrane). Hemelytra speckled 


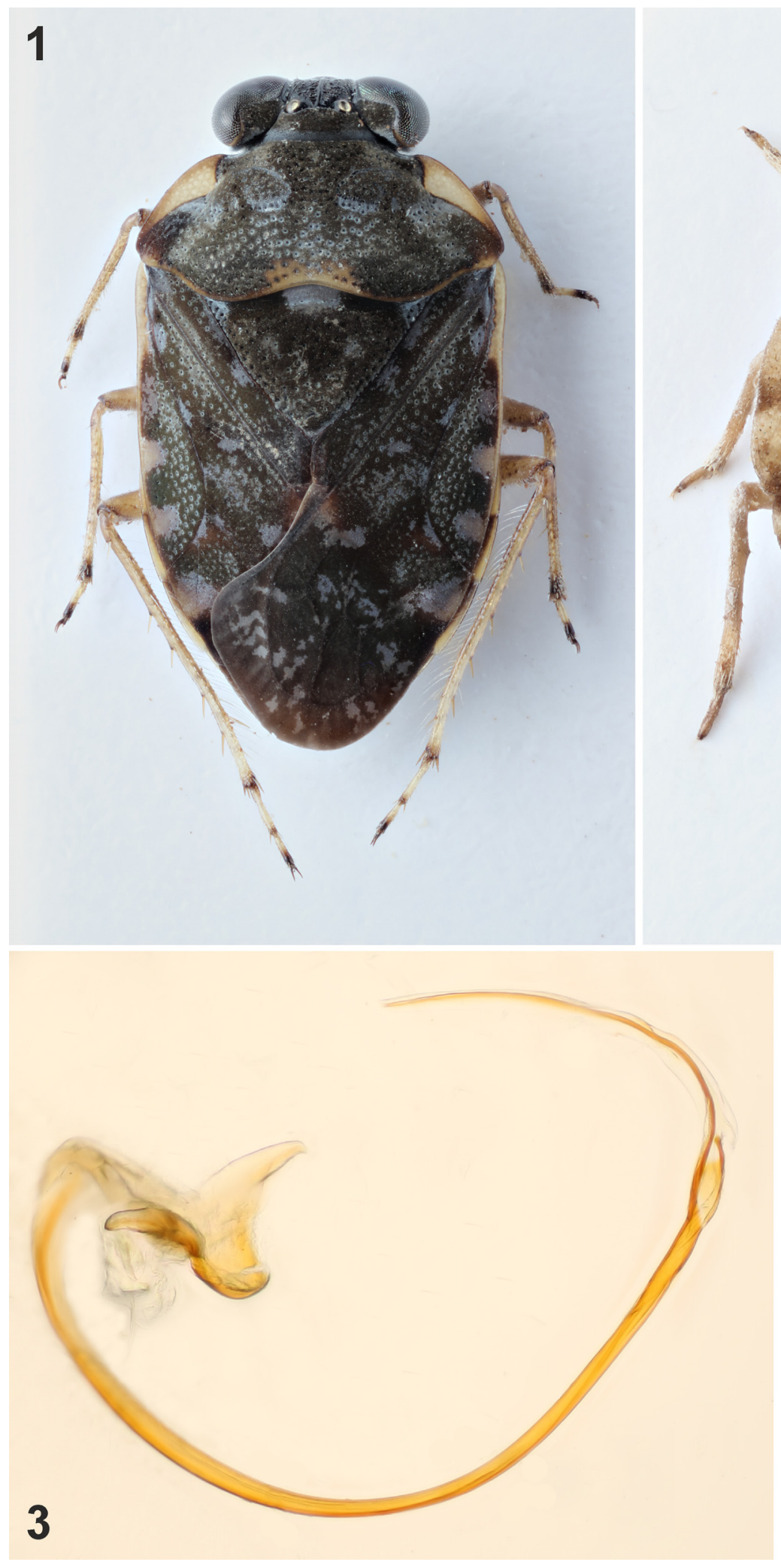

Figs 1-3. Ochterus papaceki Kment \& Carapezza sp. nov. 1 - holotype, $\widehat{\jmath}$, wadi Matyaf (body length $4.80 \mathrm{~mm}$ ); 2 - larva, Arher (body length $4.10 \mathrm{~mm}$ ); 3 - aedeagus (magnification 100×). with irregular greyish-white pruinose marks (sometimes artificially disappearing due to harsh killing or mounting method). Eyes dark brown, or with silver or metallic green shimmer. Anterior margin of frontal plate, labrum, scape and pedicel yellow. Bucculae, basiflagellum and distiflagellum black. Rostrum widely black basally and apically, median part brownish to dark brown. Body ventrally black, acetabula with yellowish margins, broad at proacetabula, narrow at meso- and metacetabula; posterolateral margin of metapleuron more or less narrowly yellowish. Hypocostal lamina yellow. Ventrites with lateral margins (all segments) and posterior margins laterally (except segment II) yellowish, more widely in posterior segments. Connexivum dorsally yellowish with blackish spots in anterolateral angles. Legs pale yellowish, with apices of femora, tibiae, and tarsal segments infuscated 

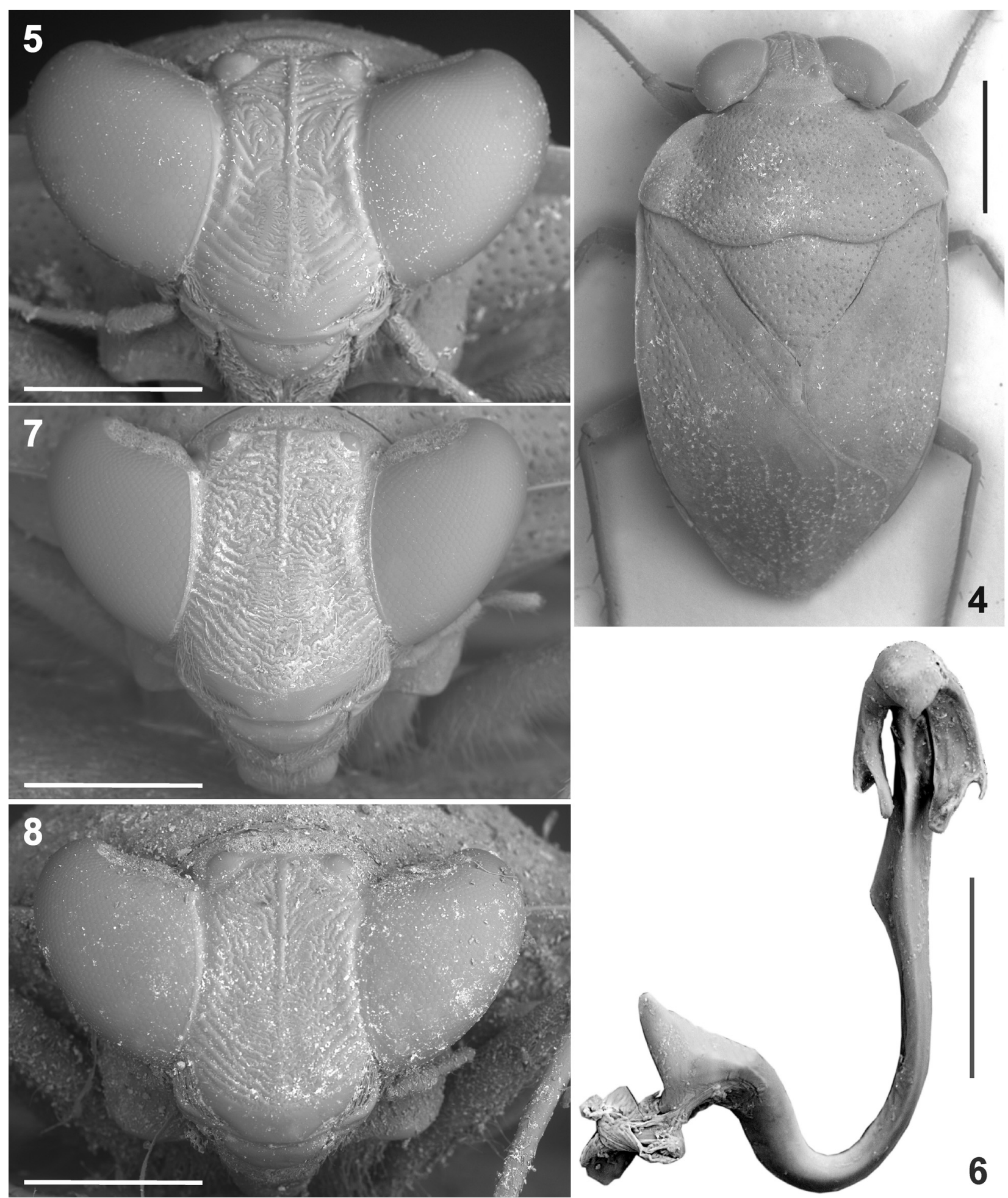

Figs 4-8. 4-6-Ochterus papaceki sp. nov., 3 , Arher: 4- body, dorsal view (magnification 27×); 5 - head, frontal view (80×); 6 - paramere, ventral view (magnification 180×). 7 - O. marginatus (Latreille, 1804), head, frontal view (75×) ( $§$, Turkey, Tunceli Prov., Ovacik env., NMPC). 8 - O. caffer (Stål,

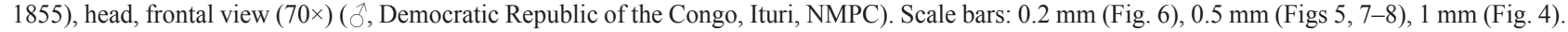

with black; dorsal margin of metatibiae with brownish line in anterior two thirds; claws black.

Structure. Body of elongate shape with lateral margins parallel-sided. Dorsal surface of body bare (Fig. 4).

Head with very large eyes (Figs 4-5), smallest interocular distance 0.33 times of head width. Vertex smooth, dull, unpunctured. Frontal plate (Fig. 5) lustrous with long median carina throughout its black portion, pos- teriorly (between ocelli and posterior margin of eyes) with distinct transverse wrinkles (forming a tree-shaped figure), medially with shallow, rather indistinct skew and transverse wrinkles, anterior portion of frontal plate with U-shaped wrinkles, the 6-7 apical ones very deep and distinct (Fig. 5). Apical yellowish portion of frontal plate smooth, without wrinkles. Anterior margin of frontal plate not produced forwards, head anteriorly rounded in lateral 
view. Scape and pedicel cylindrical, much thicker than two apical segments; basiflagellum slender, cylindrical; distiflagellum narrowly spindle-shaped. Rostrum long, reaching between metacoxae.

Thorax. Pronotum strongly transverse, width 2.3 times its median length. Lateral pronotal margins regularly rounded, only at anterolateral angles more rounded (Figs 1, 4). Yellowish flattened lateral portion of pronotum 4.3 times longer than wide (Fig. 1). Posterior margin of pronotum trisinuate. Disc of pronotum punctured, posteriorly more deeply than anteriorly (Fig. 4). Mesoscutellum triangular, convex, sparsely punctured. Hemelytron (Fig. 4) unevenly punctured: clavus with few deep punctures anteromedially, few shallow punctures posteromedially, and one row of small punctures along lateral claval margin; endocorium with scattered deep punctures in anterior half and along claval margin, rest of posterior half unpunctured; embolium with regularly dispersed deep punctures except the unpunctured costal margin; portion of corium posteriad of $\mathrm{R}+\mathrm{M}$ vein with sparse shallow punctures.

Legs. Femora unarmed, ventral surface with long, thin, whitish setae. Pro- and mesotibiae covered with short pale spines, mesotibiae also with few longer spines. Metatibiae externally with 5 long, stout, pale brownish spines and sparse, short, fine setae, internally with one long, stout, pale brownish spines subapically, and row of long, fine, brownish setae; apical margin of metatibiae with 5 long, stout, brown spines.

Male abdomen. Abdominal segments VII and VIII and male genitalia asymmetrical. Apical border line of male abdomen slightly incised posteriad of genital capsule. Genital capsule ventrally slightly keeled medially, apically produced into subquadrate process (Fig. 11) beset with long to short setae on left side (shortening towards apex) and additional row of long setae subapically on right side. Right paramere (Figs 6, 9-10) with short, widely rounded, punctate cap, ventrally pointed into short tooth (Fig. 9); both appendages rather adjacent to the shaft (Figs 6, 9-10); left appendage (Figs 6,9) more slender and slightly shorter than right one, slightly curved towards shaft; right appendage (Fig. 9) wider, slightly longer, slightly diverging laterad from shaft; both appendages apically narrowly incised, bearing microscopic granulation (best visible laterally); paramere shaft triangular in cross section, ventrally keel-like (Fig. 6), dorsally flattened, lamellate, with two rounded lateral processes, right apical one about midlength of appendages (Fig. 10) and left middle one about half-length of shaft (also visible in ventral view) (Figs 6, 9-11). Aedeagus with distal flagellum distinctly shorter than basal portion of flagellum, slender, with closely appressed phallotheca (Fig. 3).

Female abdomen symmetrical. Subgenital plate (sternite VII) strongly convex, its posterior margin trisinuate, with pair of shallow submedian insinuations and one deep and narrow median insinuation (Fig. 12).

Larva (5th instar) (Fig. 2). Body dark brown,with explanate lateral margins of pronotum, wing pads, posterolateral areas of connexival segments and legs yellowish brown. Anterior margin of frontal plate with row of 14

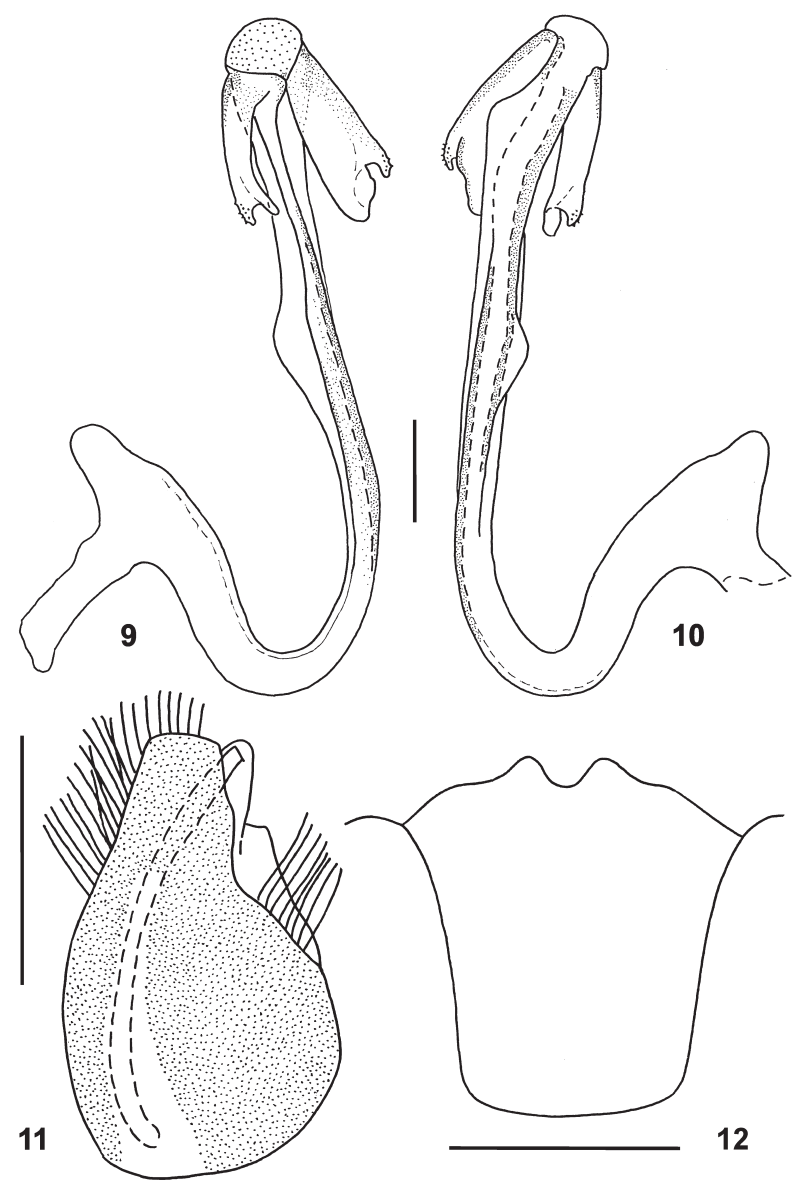

Figs 9-12. Ochterus papaceki sp. nov. 9-11 - స, wadi Matyaf: 9-10 paramere $(9$ - ventral view, 10 - dorsal view; magnification $20 \times)$; 11 - pygophore, ventral view. 12 - + , Arher, subgenital plate, ventral view. Scale bars: $0.1 \mathrm{~mm}$ (Figs 9-10), 0.5 mm (Figs 11-12).

short, stout spines, the posterior ones shortest and thinnest, anterior ones longest and stoutest. Anterolateral angles of pronotum narrowly rounded, prominent, lateral pronotal angles widely rounded. Wing pads reaching posterior margin of thorax. Head, thorax and abdominal tergites covered with short, adpressed to semierect whitish setae.

Measurements $(\mathrm{mm})$. Males: Socotra $(\mathrm{n}=10$; median (minimum-maximum)). Body length: 4.61 (4.49-4.80); head width: 1.51 (1.46-1.61); pronotum width: 2.49 (2.44-2.59); maximum width of body (ca. in corium midlength): 2.49 (2.44-2.59); length of antennal segments: scape (I) - 0.10 (0.10-0.10), pedicel (II) - 0.14 (0.14-0.16), basiflagellum (III) $-0.26(0.25-0.27)$, distiflagellum $(\mathrm{IV})-0.29(0.23-0.29)$. Tanzania $(\mathrm{n}=1)$. Body length: 4.71; head width: 1.57 ; pronotum width: 2.55 ; maximum width of body: 2.60; length of antennal segments: scape (I) -0.12 , pedicel (II) -0.15 , basiflagellum (III) -0.30 , distiflagellum (IV) -0.32 .

Females. Socotra $(n=9)$. Body length: $4.97(4.76-5.12)$; head width: 1.56 (1.51-1.56); pronotum width: 2.59 (2.54 2.63); maximum width of body (ca. in corium midlength): 2.63 (2.59-2.63); length of antennal segments: I - 0.10 (0.10-0.10), II - 0.14 (0.14-0.16), III - 0.27 (0.25-0.27), IV -0.29 (0.27-0.31). Tanzania $(\mathrm{n}=1)$. Body length: 5.20; head width: 1.60 ; pronotum width: 2.75 ; maximum 
width of body: 2.77; length of antennal segments: scape (I) -0.12 , pedicel (II) -0.15 , basiflagellum (III) -0.32 , distiflagellum (IV) -0.30 .

$5^{\text {th }}$ instar larva $(\mathrm{n}=1)$. Body length: 4.10 ; head width: 1.56; pronotum width: 2.54; maximum width of body (across wing pads): 3.02 .

Differential diagnosis. Ochterus papaceki sp. nov. resembles several other Ochterus species in which the costal margin of corium is yellowish, connected with two rounded yellowish lateral spots on embolium, and the dorsal surface of the body is bare (see KormiLev 1973; Polhemus \& Polhemus 2008, 2012; Chandra \& JehamaLAR 2012), but differs from these similar species by the unique combination of the rough striation of the frontal plate and shape of the right paramere. The apex of the head is anteriorly rounded in lateral view; the frontal plate is bearing a series of deep U-shaped wrinkles anteriorly and a tree-like figure posteriorly (Fig. 5), resembling $O$. marginatus (Latreille, 1804) (Fig. 7) and O. nicobarensis Chandra \& Jehamalar, 2012 (CHANDRA \& JEHAMALAR 2012: fig. 2), and among differently coloured species e.g. to $O$. australicus Jaczewski, 1934 (ANDERSEN \& WeIr 2004: fig. 18.4.C), but all of these species have the paramere shaft simply cylindrical and the left and right appendages of different shape (see JACZEWSKI 1937; KORMILEV 1973; Andersen \& Weir 2004; Polhemus \& Polhemus 2008, 2012; Chandra \& Jehamalar 2012). The paramere with its laterally lamellate shaft (Figs 6, 9-11) resembles that of $O$. caffer (Stål, 1855), but its lamella and appendages have different shapes, the appendages being distinctly divergent from the shaft (see JACZEWSKI 1934: figs 11-13, KoRMILEv 1973: figs 7-8); that species differs also in regard to the regularly and shallowly wrinkled frontal plate (Fig. 8). Ochterus papaceki and O. caffer also differ from $O$. marginatus by the shape of the lateral pronotal margin, which is less rounded laterally, with more rounded and therefore more prominent anterolateral pronotal angles in the first two species, while in $O$. marginatus it is strongly and regularly rounded without apparent anterolateral pronotal angles. The enigmatic $O$. strigicollis Horváth, 1913, described from Israel, differs clearly from the new species by the shape and structure of the pronotum as figured by HoRvÁth (1913: fig. 2, mislabelled as O. marginatus). Ochterus africanus Jaczewski, 1938, known only from the female holotype (Figs 14-16), differs markedly from the new species by the frontal plate being lamellate anteriorly, and protruding forwards (see JACZEWSKI 1938: figs 1-2).

Etymology. We dedicate this species to our dear colleague, professor Miroslav Papáček (1953-2019), an eminent specialist in the morphology, taxonomy, bionomics and ecology of aquatic and semiaquatic bugs (see Ditrich et al. 2020). Mirek was my (P. Kment) first tutor and supervisor in Heteroptera during my master studies and I will always remain indebted to him for all of his advice and kind help.

Habitat. At Arher the species was collected by hand or by suction sampler from wet places with short-grazed grasses on sand, surrounding a small brook situated among sand dunes and groups of Tamarix nilotica (Ehrenb.) Bunge about $50 \mathrm{~m}$ from sea coast (P. Kment,

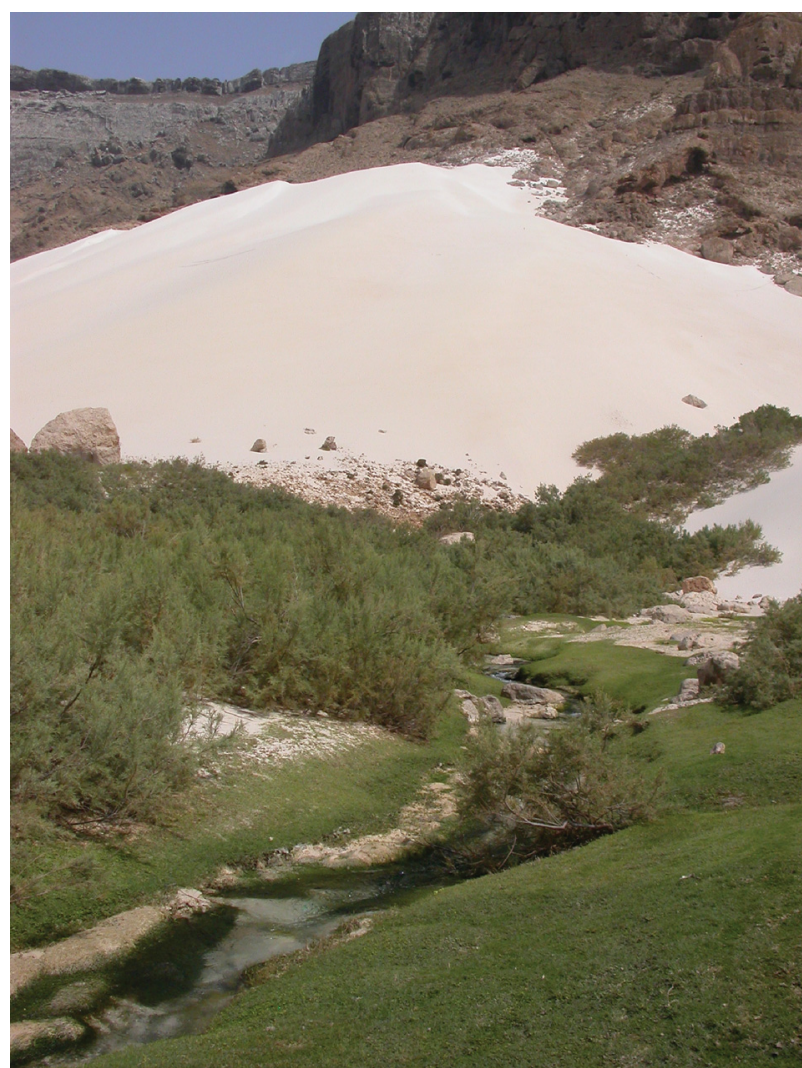

Fig. 13. Socotra, Halla Arher, habitat of Ochterus papaceki sp. nov.

pers. observ.; see Fig. 13). In Wadi Matyaf the species was collected by hand on the wet sandy banks of the estuarine area of the river, about $800 \mathrm{~m}$ far from sea coast (A. Carapezza, pers. observ.). Both localities belong to the coastal zone of Socotra (0-200 m a.s.1., see Brown \& Mies 2012).

\section{Key to Ochterus species of Afrotropical Region}

(Modified from Kormilev (1973) and Polhemus \& Polhemus (2008))

1 Frontal plate apically produced, forming sharp lamella (best seen in lateral view) (Figs 15-17; JACZEWsKI 1938: figs 1-2). Central African Republic.

O. africanus Jaczewski, 1934

- Frontal plate apically not produced, bluntly rounded in lateral view.

2 Body covered with more or less erect stiff bristles, venter covered with dense, inclined, and long hairs (KoRMILEV 1973: figs 9-10). Striation of the frontal plate sharp, particularly on the upper half of the plate. Right paramere with its shaft lamellate (KoRMILEV 1973: fig. 11). Angola. ....................... O. piliferus Kormilev, 1973

- Body bare, without more or less erect stiff bristles. ... 3

3 Hemelytra usually with three irregular or semicircular yellowish spots along costal margin of corium (sometimes overlain with pruinose spots). Europe, Africa or Asia.

- Hemelytra without yellowish spots along costal margin of corium, but with irregular pruinose spots (sometimes weakly formed). Seychelles or Mascarene Islands. 
4 Shaft of right paramere essentially cylindrical. ............ 5

- Shaft of right paramere flattened, lamellate laterally. ....

5 Striation of the frontal plate sharp, particularly on the upper half of the plate (Fig. 7). Right paramere figured e.g. in JaCzewski (1934: figs 6-10), Kormilev (1973: figs 2-3) and Polhemus \& Polhemus (2012: figs 5-7). $S$ Europe, Africa, Asia.

O. marginatus marginatus Latreille, 1804

- Striation of the frontal plate fine, particularly on the upper half of the plate (Kormilev 1973: fig. 4). Right paramere figured in Kormilev (1973: fig. 5). Tropical Africa. O. minor Kormilev, 1973

6 Striation of the frontal plate sharp, particularly on the upper half of the plate with distinct tree-shaped figure (Fig. 5). Lateral appendages of right paramere adjacent to the shaft, shaped as on Figs 6, 9-10. Socotra, Tanzania. .............. O. papaceki Kment \& Carapezza sp nov.

- Striation of the frontal plate fine, particularly on the upper half of the plate (Fig. 8). Lateral appendages of right paramere distinctly divergent from the shaft, shaped as figured in JACZEWSKI (1934: figs 11-13) and Kormilev (1973: fig. 5). Tropical Africa and Madagascar.

O. caffer Stål, 1855

7 Posterior part of endocorium with obvious punctures. Right paramere figured in Polnemus \& Polhemus (2008: figs 2-4). Mauritius.

....... O. stysi D. A. Polhemus \& J. T. Polhemus, 2008

- Posterior part of endocorium without, or with very weak punctures. Right paramere figured in PolHemus (1992a: fig. 3) and Polhemus \& Polhemus (2013: figs 47-48). Seychelles.

O. seychellensis D. A. Polhemus, 1992

\section{Taxonomic catalogue of modern species of the family Ochteridae}

\section{Superfamily OCHTEROIDEA Kirkaldy, 1906 [1815]}

Ochteridæ Kirkaldy, 1906a: 149 (according to principle of coordination). Ochteroïdeae: REUTER (1910): 60 (as superfamily). Gelastocoroidea: PopOv (1971): 98 (as superfamily) Ochteroidea: ŠTYS \& JANSSON (1988): 7, 22 (clarification of nomenclature).

\section{Family OCHTERIDAE Kirkaldy, 1906 [1815]}

Pelogonida Leach, 1815: 123 (diagnosis). Type genus: Pelogonus Latrei1le, 1809 [= Ochterus Latreille, 1807].

Pelegonidae (incorrect subsequent spelling): Puton (1869): 38 (list).

Pelogonidae: Dohrn (1859): 52 (list).

Pelogoniidae (incorrect subsequent spelling): China \& Miller (1955): 267 (nomenclature).

Ochteridæ Kirkaldy, 1906: 149 (new substitute name for Pelogonidae). Type genus: Ochterus Latreille, 1807.

Ochteridae: China \& Miller (1959): 16 (nomenclature); Polhemus \& Polhemus (1988a): 542 (nomenclature); ŠTYs \& JANSSON (1988): 7, 22 (nomenclature).

Note. Ochteridae is an unnecessary new substitute name for Pelogonidae, but having gained general acceptance it takes the date of the replaced name and is deemed to be its senior synonym (ICZN 1985: Art. 40b, Recommend. 40A) (Polhemus \& Polhemus 1988a: 542, S̆TYs \& JANSSON 1988: 7).

\section{Genus Megochterus Jaczewski, 1934}

Megochterus Jaczewski, 1934: 610-612 (description, differential diagnosis). Type species: Pelogonus nasutus Montandon, 1898 by original designation.

Megochterus: JACZEWSKI (1935): 482 (key to genera and species); SCHELL (1943a): 30 (key to genera); KormileV (1971): 443 (diagnosis); ŠTYS \& JANSSON (1988): 7 (catalogue); BAEHR (1989): 112 (key to genera); BAEHr (1990a): 198-202 (key to species, revision); CAssis \& Gross (1995): 259 (catalogue); ANDERSEN \& WEIR (2004): 264-265, 335 (key to genera, diagnosis, checklist); CHEN et al. (2005): 78 (key to genera); YAO et al. (2007): 828, 830 (key to genera, differential table); YAO et al. (2011): 590, 597-598 (key to genera, phylogeny); Melo (2015): 344 (key to genera, diagnosis).

\section{Megochterus nasutus (Montandon, 1898)}

Pelogonus nasutus Montandon, 1898: 72-73 (original description, differential diagnosis). Lectotype: $q$ (designated by BAEHR 1990a: 198), 'Australia' (MNHN).

Megochterus nasutus: JACZEWSKI (1934): 610-613 (redescription, illustrations); JACZEWSKI (1935): 482 (key to species); KoRMILEV (1971): 434, 443-444 (partim, specimens from Queensland; redescription, illustrations); BAEHR (1990a): 198-201 (key to species, redescription, illustrations, lectotype designation); CAssis \& Gross (1995): 259 (catalogue); ANDERSEN \& WEIR (2004): 264-265, 335 (key to species, illustrations, checklist); CHEN et al. (2005): 80 (illustration); PoPOV \& HeIss (2014a): 188 (illustration).

Distribution. Australian Region: Australia: New South Wales (BAEHR 1990a), Queensland (KoRMILEv 1971; BAEHR 1989, 1990a; ANDERSEn \& WeIr 2004), South Australia (Andersen \& Weir 2004), Tasmania (BAehr 1990a, AnDERSEN \& WEIR 2004).

\section{Megochterus occidentalis Baehr, 1990}

Megochterus nasutus: KoRMILEv (1971): 444 (partim, specimen from Kelmscott, Western Australia).

Megochterus occidentalis Baehr, 1990a: 198-202 (key to species, description, illustrations). Ноцотуре: $\hat{\jmath}$, Australia: Western Australia: ' 3 km N.E. Broke Inlet, E. of Walpole' (WAMA).

Megochterus occidentalis: CAssis \& Gross (1995): 259 (catalogue); ANDERSEN \& WeIr (2004): 264-265, 335 (key to species, illustrations, checklist).

Distribution. Australian Region: Australia: Western Australia (BAEHR 1990a, ANDERSEN \& WeIR 2004).

\section{Genus Ochterus Latreille, 1807}

Ochterus Latreille, 1807: 142-143 (original description). Type species: Acanthia marginata Latreille, 1804 [= Ochterus marginatus], by monotypy.

Ochtherus Agassiz, 1847: 254. Unjustified emendation, junior objective synonym of Ochterus Latreille, 1807.

Ochtherus (incorrect subsequent spelling): BERGROTH (1890a): lxvi (nomenclature).

Ochthera (incorrect subsequent spelling): BERGROTH (1890a): 1xvi (nomenclature).

Octhera (incorrect subsequent spelling): BERGROTH (1890b): cxix (nomenclature).

Octherus (incorrect subsequent spelling): BERGROTH (1890b): cxix (nomenclature).

Ochterus: KolEnATI (1857): 453-454 (redescription); KiRKALDY (1906): 149 (catalogue); KirKaLdy \& Torre BuEno (1909): 179 (catalogue); OSHANin (1912): 89 (catalogue); BARBer (1913): 213-215 (key 
to species, taxonomy); VAN DuZEe (1917): 475-476 (catalogue); Blatchley (1926): 1020-1022 (key to species, review); JACZEWSKI (1934): 601-610 (diagnosis, revision); JACZEWSKI (1935): 482 (key to genera and species); Wu (1935): 557 (catalogue); SCHELl (1943a): 30-36 (key to genera, revision); Schell (1943b): 37-47 (revision); Chapman (1958): 119-120 (key to species); Kormilev (1971): 433-443 (revision); NiESER (1975): 26 (key to genera and species); Menke (1979): 124-125 (catalogue); Froeschner (1981): 61 (key to genera); ŠTYS \& JANSSON (1988): 8 (catalogue, nomenclature); BAEHR (1989): 112-125 (key to species); BAEHR (1990b): 450-476 (diagnosis, key to species, revision); CAssis \& Gross (1995): 259-262 (catalogue); Polnemus (1995a): 25 (catalogue); ANDERSEN \& WeIr (2004): 264, 266-271, 335-336 (key to genera, diagnosis, checklist); CHEN et al. (2005): 78, 423 (key to genera, checklist); EPLER (2006): 1.15, 12.1-12.4 (key to species); YAO et al. (2007): 828, 830 (key to genera, differential table); MAzzuccoNI et al. (2009a): 212-213 (key to genera, illustration); HECKMAN (2011): 457-463 (key to genera and species); YAO et al. (2011): 590, 597-598 (key to genera, phylogeny); Polnemus \& Polhemus (2014): 164 (key to genera); BARBosA \& Rodrigues (2015): 163, 166, 174, 176, 182 (key to genera, illustrations); LLANO \& GUTIÉRREZ (2015): 378 (distribution, illustrations); Melo (2015): 344 (key to genera, diagnosis); MoreIra et al. (2019): 179, 182, 188, 190 (key to genera, illustrations).

Pelogonus Latreille, 1809: 384 (new substitute name). Junior objective synonym; unnecessary new substitute name for Ochterus Latreille, 1809 , proposed because of presumed homonymy with Ochthera Latreille, [1802] (Diptera)

Pélogone (vernacularization): LATREILle (1825): 423

Pelegonus (incorrect subsequent spelling): LAPORTE (1832): 6, 13 (diagnosis); Puton (1869): 38 (list); Puton (1880): 209 (diagnosis).

Pelogonus: Le Peletier \& Serville (1825): 33 (redescription); Dufour (1833): 192-193 (comparative notes); BuRMEISTER (1835): 202-204 (key to genera, redescription); BRULLÉ (1836): 276-277 (differential diagnosis); Blanchard (1840): 93-94 (redescription); АмYOT \& SERVILle (1843): 407-409 (redescription); Herrich-SCHÄFFER (1849): 18, 23-24 (key, redescription); FIEBER (1851): 14-15 (redescription); FIEBER (1861): 103 (redescription, catalogue); STÅL (1866): 170 (redescription); CHAMPION (1901): 344-346 (key to species, revision); Distant (1906): 13-14 (catalogue, redescription); OshanIN (1909): 955 (catalogue).

Neochterus Mahner, 1993: 92, 191 (as subgenus of Ochterus). Type species: Ochterus manni Hungerford, 1927, original designation. Unavailable name.

Neochterus: Polhemus (1995a): 25 (listed as valid); CiAnFeroni (2012): 62 (listed as valid); Barbosa \& Rodrigues (2015): 174 (listed as valid).

Note. MAHNer (1993) established this presumably paraphyletic taxon to accommodate American species of Ochterus. However, this taxon was defined merely as opposite to Palaeochterus Mahner, defined by its apomorphic structure of the right paramere, and its geographic distribution, without providing a single morphological character, which renders Neochterus Mahner an unavailable name according to Articles 13.1 (ICZN 1999) (P. Štys, in litt., as well as our opinion).

Palaeochterus Mahner, 1993: 92, 191 (as subgenus of Ochterus). Type species: Acanthia marginata Latreille, 1804, original designation. Junior objective synonym of Ochterus Latreille, 1807, recognized by Andersen (1995: 161) and Polhemus (1995a: 25).

Note. MAHNER (1993) established this taxon for Old World species of Ochterus, which he considered a monophyletic taxon based on the characteristic right paramere with rounded capitulum and two subapical appendages. Palaeochterus Mahner is thus an available name according to Articles 13.1 and 13.3 (ICZN 1999), but its author ignored the principle of coordination and created thus a junior objective synonym of Ochterus, as both taxa share the same type species, Acanthia marginata Latreille, 1804 (ICZN 1999: Art. 43.1).

Note. Llano \& GutiÉRrez (2015) provided records and habitus photographs of two unidentified Ochterus species from Colombia.

\section{Ochterus acutangulus (Champion, 1901)}

Pelogonus acutangulus Champion, 1901: 345-346, Pl. XX: fig. 11 (key, original description, illustration). Holotype: + , Guatemala: 'Rio Naranjo' (BMNH).

Ochterus acutangulus: KirKALDY \& TORRE BuENo (1909): 179 (catalogue); Barber (1913): 214 (key to species); Schell (1943b): 38-39 (original description repeated); ? DRAKE (1952): 73-74 (checklist, comparative note, illustration); Alayo (1971): 4-5 (key to species); Alayo (1974): 34-35 (key to species); Heckman (2011): 459-460 (key to species, illustration); Polnemus \& Polnemus (2016), 17-20 (key to species, redescription, illustrations).

Distribution. Neotropical Region: Central America: Belize (Polhemus \& Polhemus 2016); Guatemala (ChamPion 1901, Kirkaldy \& Torre Bueno 1909, Drake 1952, Polhemus \& Polhemus 2016); Mexico (Drake 1952): Chiapas (Polmemus \& Polnemus 2016).

Notes. Comprative notes and record of $O$. acutangulus from Mexico: Tejupilco by Schell (1943b) actually refers to O. schellae (see DrAKE 1952), also her records from Mexico: Veracruz and Panama: Porto Bello (ScHell 1943b) seem doubtful and require revision. DraKe (1952: 74) provided an illustration of a female from Cuba. The distribution in Cuba was accepted by Alayo (1971, 1974). However, there is no other record from Cuba (MuÑoz Riviaux et al. 2010a,b; NARANJO et al. 2010), and its presence on the island is considered doubtful by PoLHEMus $\&$ Polhemus (2016).

\section{Ochterus aeneifrons aeneifrons (Champion, 1901)}

Pelogonus marginatus (misidentification, partim): UHLER (1893): 706 (list, 'two forms'); Uhler (1894): 222 (variability).

Pelogonus ceneifrons Champion, 1901: 344-345-346, Pl. XX: figs 12, 13, 13a (key, original description, variability, llustrations). SYNTYPES: 24 spec. ( $\left.\delta \delta q_{+}\right)$, Mexico: Tabasco: 'Teapa'; Guatemala: 'San Gerónimo, Guatemala city'; Panama: 'Tolé, Peña Blanca, San Feliz'; Grenada; St. Vincent (BMNH).

Ochterus ceneifrons: KiRKALDY \& TORRE BuENO (1909): 179 (catalogue).

Ochterus cenifrons (incorrect subsequent spelling): BARBER (1913): 213 (key to species).

Ochterus aenifrons (incorrect subsequent spelling): ScHELL (1943a): 32, 35 (key to species, illustration); Schell (1943b): 43-44 (original description repeated, differential diagnosis); DraKe (1952): 74 (checklist).

Ochterus aeneifrons: Froeschner (1981): 61 (catalogue); FroeschNER (1999): 148 (catalogue); Heckman (2011): 462 (key to species); Cianferoni (2012): 67 (key to species for Ecuador).

Ochterus aeneifrons aeneifrons: NIESER \& ALKINs Koo (1991): 14 (key); Polhemus \& Polhemus (2012): 17, 20-24 (key to species, redescription, illustrations).

Ochterus barberi (misidentification): ScHELL (1943b): 42 (paratypes from Orizaba, Mexico) (see Polhemus \& Polhemus 2016).

Distribution. Neotropical Region: Central America: Belize (Polhemus \& Polhemus 2016); Costa Rica (Schell 1943b, Polhemus \& Polhemus 2016); El Salvador (Polhemus \& Polhemus 2016); Guatemala (Champion 1901, Kirkaldy \& Torre Bueno 1909, Schell 1943b, Polhemus \& Polhemus 2016); Honduras (Schell 1943b); Mexico (Kirkaldy \& Torre Bueno 1909, Schell 1943b, Drake 1952): Chiapas (Polmemus \& Polhemus 2016), Colima (Polnemus \& Polhemus 2016), Guerrero (Polhemus \& Polhemus 2016), Jalisco (Polhemus \& Polhemus 2016), Mexico (Polnemus \& Polhemus 2016), Nayarit (Polnemus \& Polhemus 2016), Nuevo León (Polhemus \& Polhemus 
2016), Oaxaca (Polhemus \& Polhemus 2016), Puebla (Polhemus \& Polhemus 2016), Queretaro (Polhemus \& Polhemus 2016), San Luis Potosí (Polhemus \& Polhemus 2016), Tabasco (Champion 1901), Tamaulipas (Polhemus \& Polnemus 2016), Veracruz (Polhemus \& Polhemus 2016); Nicaragua (Polhemus \& Polhemus 2016); Panama (Champion 1901, Kirkaldy \& Torre Bueno 1909, Schell 1943b, Froeschner 1999, Polhemus \& Polhemus 2016). Caribbean Islands: Grenada (Uhler 1894, as P. marginatus; Champion 1901; Kirkaldy \& Torre Bueno 1909); St. Vincent (Uhler 1893, as P. marginatus; CHAMPION 1901; Kirkaldy \& Torre Bueno 1909); Trinidad and Tobago (Kirkaldy \& Torre Bueno 1909, Schell 1943b, Nieser \& Alkins Koo 1991, Polnemus \& Polhemus 2016). South America: Colombia (Schell 1943b, Drake 1952, Polhemus \& Polhemus 2016); Ecuador (Schell 1943b; Froeschner 1981; NiESER \& CHEN 1992, as O. aeneifrons).

Note. The subspecies identity of the specimens recorded from Ecuador only as $O$. aeneifrons by NIESER \& CHEN (1992) requires confirmation.

\section{Ochterus aeneifrons surinamensis Nieser, 1975} (Fig. 14)

Ochterus aeneifrons surinamensis Nieser, 1975: 26-27-28, 31, Pl. 2b (key to species, description, illustrations). Hоцотуре: $\hat{\jmath}$, Suriname: Carolinakreek, upstream of bridge (UM $\rightarrow$ ZMAN $\rightarrow$ RMNH)

Ochterus aeneifrons surinamensis: HECKMAN (2011): 458, 462 (diagnosis, illustration); MoreIra et al. (2011): 58 (checklist Brazil); CORDEIRO et al. (2014): 67 (key to species for Brazil, illustration); BARBOSA \& RoDRIGUES (2015): 182 (illustrations).

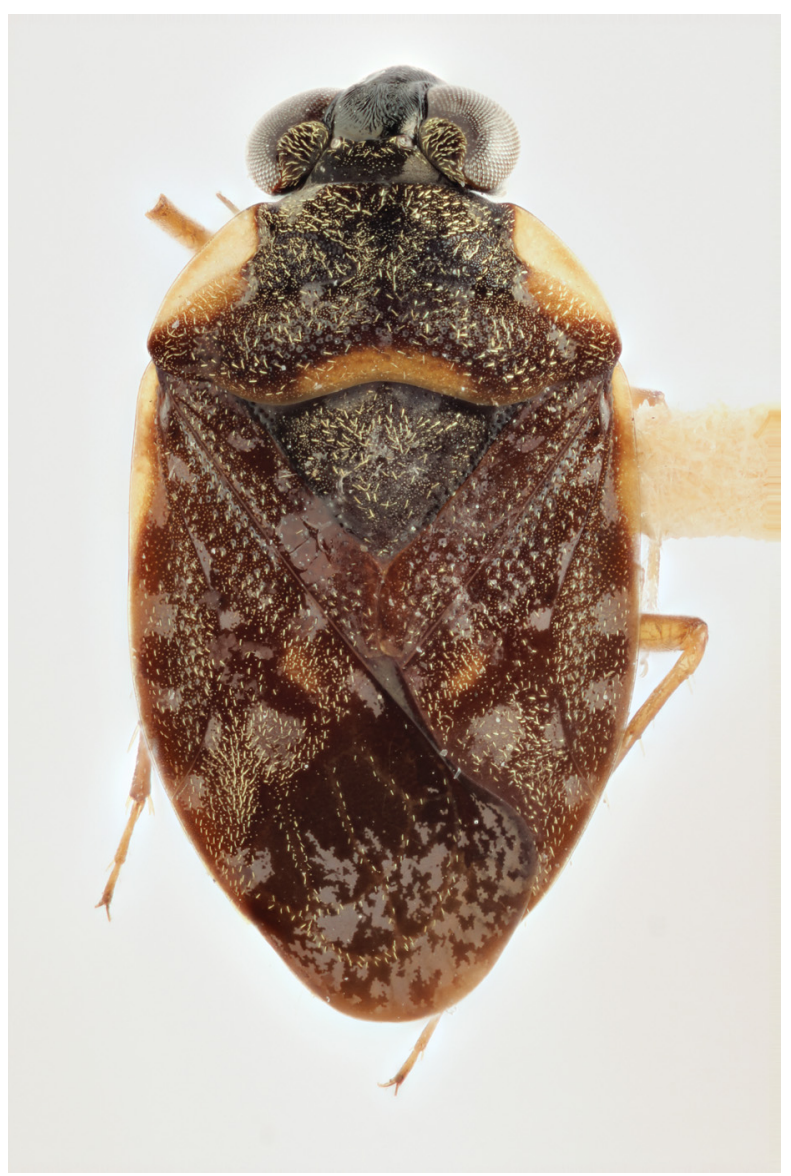

Fig. 14. Ochterus aeneifrons surinamensis Nieser, 1975, female, Colombia: Rio Frio, $4.51 \mathrm{~mm}$.
Material examined. COLOMBIA: Cundinamarca: Rio Frio, 15.iii.1925, 2 + (Fig. 14), C. J. Drake det. as O. aeneifrons, P. Kment revid. (NMPC).

Distribution. Neotropical Region: South America: Brazil: Amazonas (Pereira \& Melo 2007, Moreira et al. 2011, Cordeiro et al. 2014), Minas Gerais (Pelli et al. 2006, Moreira et al. 2011, Cordeiro et al. 2014); Colombia (new record), Suriname (NIESER 1975); Venezuela (DraKe 1952, as O. aeneifrons; Polnemus \& Polnemus 2016).

Note. The holotype of the species was originally deposited in Zoölogisch Museum der Rijksuniversiteit, Utrecht. Later, its insect collection was transferred to ZMAN, which was later merged with RMNH. However, N. Nieser recently failed to find the holotype in RMNH (N. Nieser, pers. comm.).

\section{Ochterus africanus Jaczewski, 1938} (Figs 15-17)

Ochterus africanus Jaczewski, 1938: 186-187 (description, differential diagnosis, illustrations). Holotype (Figs 14-16): 1 \% , Central African Republic: 'Französisch Kongo, Fort Crampel' (ZMUH).

Ochterus africanus: KormiLev (1973): 2 (list, incertae sedis).

Distribution. Afrotropical Region: Central African Republic (JACZEWSKI 1938).

Note. The photographs of the holotype of $O$. africanus (Figs 15-17) are presented here for the first time.

\section{Ochterus alticola Baehr, 1990}

Ochterus alticola Baehr, 1990b: 451, 453, 455-459, 461, 473 (key to species, description, illustrations). Ноготуре: 0 , Papua New Guinea: 'Mafula, $4000 \mathrm{ft}$. [= $1219 \mathrm{~m}$ a.s.1.]' (BMNH).

Ochterus alticola: CHEN et al. (2005): 413 (checklist).

Distribution. Australian Region: Papua New Guinea (BAEHR 1990b).

\section{Ochterus americanus (Uhler, 1876)}

Pelogonus americanus Uhler, 1876: 335-336 (original description). Syntypes: USA: 'Texas, Illinois, Eastern Massachusetts, Pennsylvania' (USNM).

Pelogonus americatus (incorrect subsequent spelling): SNow (1906): 180 (distribution).

Ochterus americanus: Torre Bueno (1906): 50 (list); KirKaLdy \& Torre Bueno (1909): 179 (catalogue); Barber (1913): 213 (key to species); VAN DuZee (1917): 476 (catalogue); Hungerford (1919): 51, 53-54, pl. III: fig. 9 (key to species, original description repeated); BLATCHLEY (1926): 1020-1021 (key to species, redescription, illustration); SCHELL (1943a): 32, 35 (key to species, illustration); Schell (1943b): 42-43 (original description repeated, differential diagnosis); DraKe (1952): 74 (checklist); Chapman (1958): 119 (key to species); WiLson (1958): 154 (key to species); BoвB (1974): 17, 20 (key to species); BENNETT \& Cook (1981): 35 (diagnosis, illustrations); Polhemus \& Polhemus (1988a): 542 (catalogue); EPLer (2006): 12.3-12.4 (key to species, illustrations); HeCKMAN (2011): 461 (key to species, illustration); Hoffman (2012): 58-59 (illustration, distribution); Polhemus \& Polhemus (2016): 16, 24-26 (key to species, diagnosis, illustrations).

Ochterus americanum (incorrect subsequent spelling): BARBER (1913): 215.

Distribution. Nearctic Region: Canada: Ontario (MAw et al. 2000); USA: ? Arizona (SNOw 1906, KirKALDY \& Torre Bueno 1909, Blatchley 1926, Polhemus \& Polhemus 1988a), Florida (BArber 1914, Blatchley 1926, ? CHAPMAN 1958, Polhemus \& Polhemus 2016), Georgia (Polhemus \& 

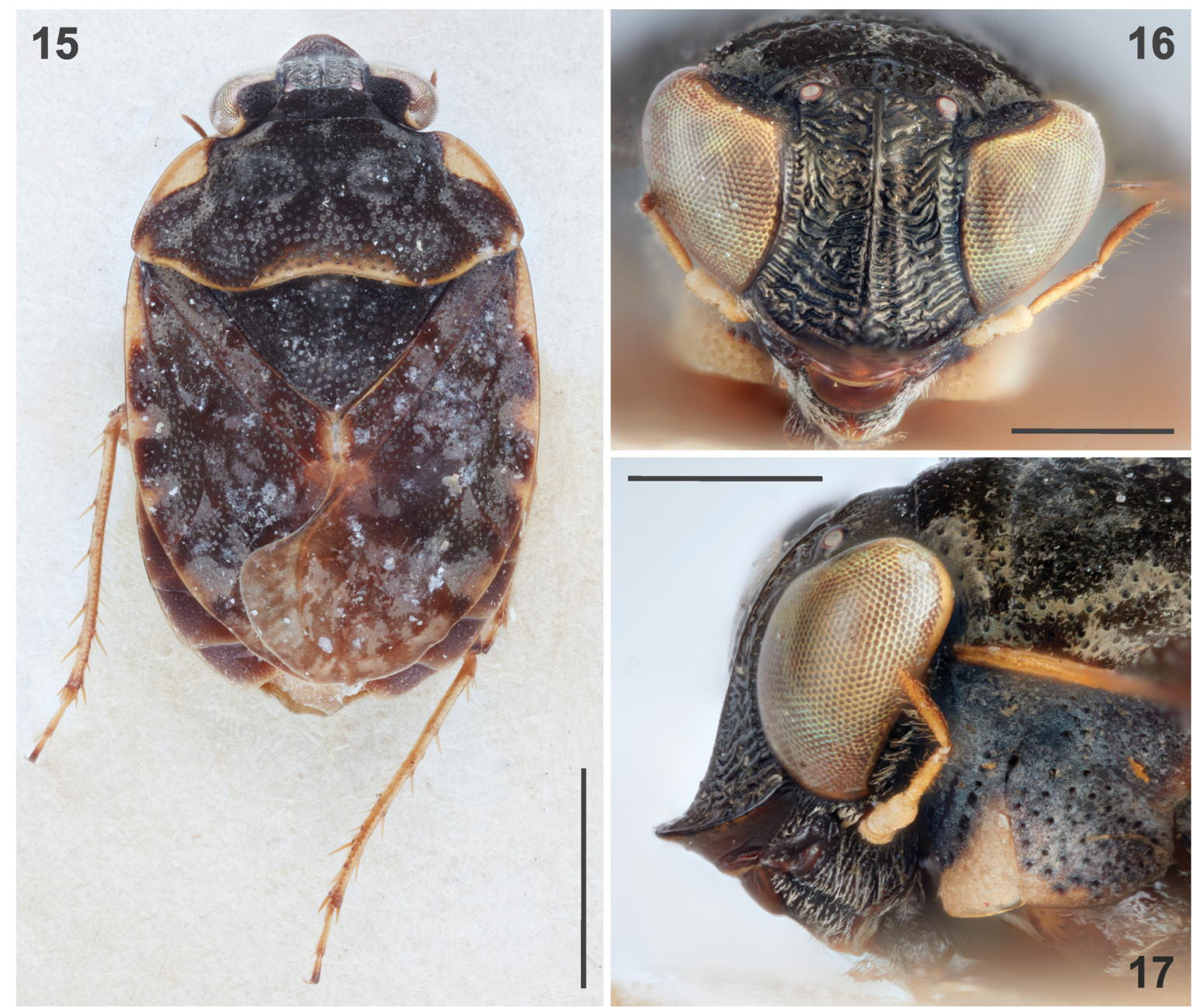

Figs 15-17. Ochterus africanus Jaczewski, 1938, female, holotype: 15 - habitus in dorsal view; 16 - head, frontal view; 17 - head, lateral view. Scale bars: $15-1 \mathrm{~mm}, 16-17-0.5 \mathrm{~mm}$. Photographs by V. Hartung.

Polhemus 2016), Illinois (Uhler 1876; KirKaldy \& Torre Bueno 1909; Blatchley 1926; Schell 1943b; Drake 1952; Polhemus \& Polhemus 1988a, 2016; Tinerella et al. 2009), Iowa (Drake 1952), Kansas (Hungerford \& BeAmer 1925; Blatchley 1926; Polhemus \& Polhemus 1988a, 2016), Louisiana (Polhemus \& Polhemus 1988a, 2016), Maryland (Kirkaldy \& Torre Bueno 1909; Drake 1952; Polhemus \& Polhemus 1988a, 2016), Massachusetts (Uhler 1876, Kirkaldy \& Torre Bueno 1909, Blatchley 1926, Johnson 1930, Drake 1952, Polhemus \& Polhemus 1988a), Minnesota (Polhemus \& Polhemus 2016), Mississippi (Schell 1943b, Drake 1952, Wilson 1958, Polhemus \& Polhemus 1988a), Missouri (Polhemus \& Polhemus 1988a, 2016; Epler 2006), Nebraska (KirKaldy \& Torre Bueno 1909; Blatchley 1926; Schell 1943b; DraKe 1952; Polhemus \& Polhemus 1988a, 2016), New Jersey (Smith 1890; Torre Bueno 1905, 1908; Kirkaldy \& Torre Bueno 1909; Blatchley 1926; Chapman 1959; Polnemus \& Polhemus 1988a, 2016), New York (Torre Bueno 1905, 1908; Kirkaldy \& Torre Bueno 1909; Blatchley 1926; Leonard 1928; Schell 1943b; Polhemus \& Polhemus 1988a), North Carolina (Torre Bueno \& Brimley 1907; Kirkaldy \& Torre Bueno 1909; Polhemus \& Polhemus 1988a, 2016), South Carolina (EPler 2006), Pennsylvania (Uhler 1876, WirTner 1904, Kirkaldy \&
Torre Bueno 1909, Polhemus \& Polhemus 1988a), Texas (Uhler 1876, Kirkaldy \& Torre Bueno 1909, Blatchley 1926, Polnemus \& Polhemus 1988a), Virginia (Polmemus \& Polhemus 1988a, Hoffman 2012).

Notes. According to T. Henry (pers. comm.), the USNM collection contains four of what appear to be syntypes of $P$. americanus Uhler: $1 q$ and one broken specimen (abdomen missing) from 'Mass.'; 1 from 'N. Ill.'; and 1 from 'Dedham, Mass, Apr. 30, 65, nr water,' as mentioned in the original description.

UhLER (1876) included also a specimen from Cuba, writing that: 'has the spots of the costal margin of the hemelytra almost obsolete; but it agrees with the United States from in all other respects'. It was listed from Cuba also by Blatchley (1926) and Heckman (2011), but Drake (1952) and later Cuban authors (AlAYo 1971, 1974; MuÑOZ RiviAuX et al. 2010a,b; NARANJO et al. 2010) did not confirm its presence in that island.

According to Polhemus \& Polhemus (1988a) records from Florida by Drake and from South Carolina (RoBACK 1958) require confirmation; the Florida specimens determined by Drake as $O$. americanus and reexamined by Polnemus \& Polhemus (1988a) were actually O. banksi. The South Carolina distribution was confirmed by EPLER (2006). 


\section{Ochterus atridermis Baehr, 1989}

Ochterus atridermis Baehr, 1989: 114, 116, 118, 122-123 (key to species, description, illustrations). HоLотуре: $\hat{\jmath}$, Australia: Queensland: 'Beerway [= Beerwah], S. E. Qlnd.' (QMBA).

Ochterus atridermis: BAEHR (1990b): 18, 453-454, 456, 459, 461, 467 (key to species, redescription, illustrations).

Ochterus atridermis: CASSIS \& GROSS (1995): 260 (catalogue); ANDERSEN \& WeIR (2004): 268, 335 (key to species, checklist).

Distribution. Australian Region: Australia: Northern Territory (BAEHR 1989, ANDERSEN \& WeIR 2004), Queensland (BAEHr 1989, ANDERSEN \& Weir 2004).

\section{Ochterus australicus Jaczewski, 1934}

Ochterus marginatus (misidentification): DisTANT (1920): 164 (specimen from Gondé, see JACZEWSKI 1934: 609).

Ochterus australicus Jaczewski, 1934: 607-610 (description, differential diagnosis, illustration) (partim, see Note). Lестотуре: $\delta$ (designated by BAeHr 1990b: 452), New Caledonia (BMNH).

Ochterus australicus (partim): KORMILEV (1971): 434, 439-442 (partim; redescription based on specimens from Queensland: North Stradbroke Island, illustrations).

Ochterus australicus: JACZEWSKI (1935): 482 (key to species); BAEHR (1989): 113, 114-116, 118 (key to species, redescription, illustrations); BAEHR (1990b): 452-456, 459, 461 (key to species, redescription, illustrations, lectotype designation); CAssis \& GRoss (1995): 260 (catalogue); ANDERSEN \& WeIr (2004): 267-270, 335 (key to species, illustrations, checklist); CHEN et al. (2005): 413 (checklist); DAMGAARD \& ZetTel (2014): 227, 236 (checklist).

Material examined.AUSTRALIA: New South WALES: Bellinger River N.P., Darkwood Rd., W of Darkwood Bridge, $30^{\circ} 26.80^{\prime} \mathrm{S} 152^{\circ} 37.12^{\prime} \mathrm{E}$, 95 m a.s.1., 27.xi.2014, 2 §̊̊, D. Rédei \& Q. Xie lgt., D. Rédei 2016 det., P. Kment revid. (NMPC).

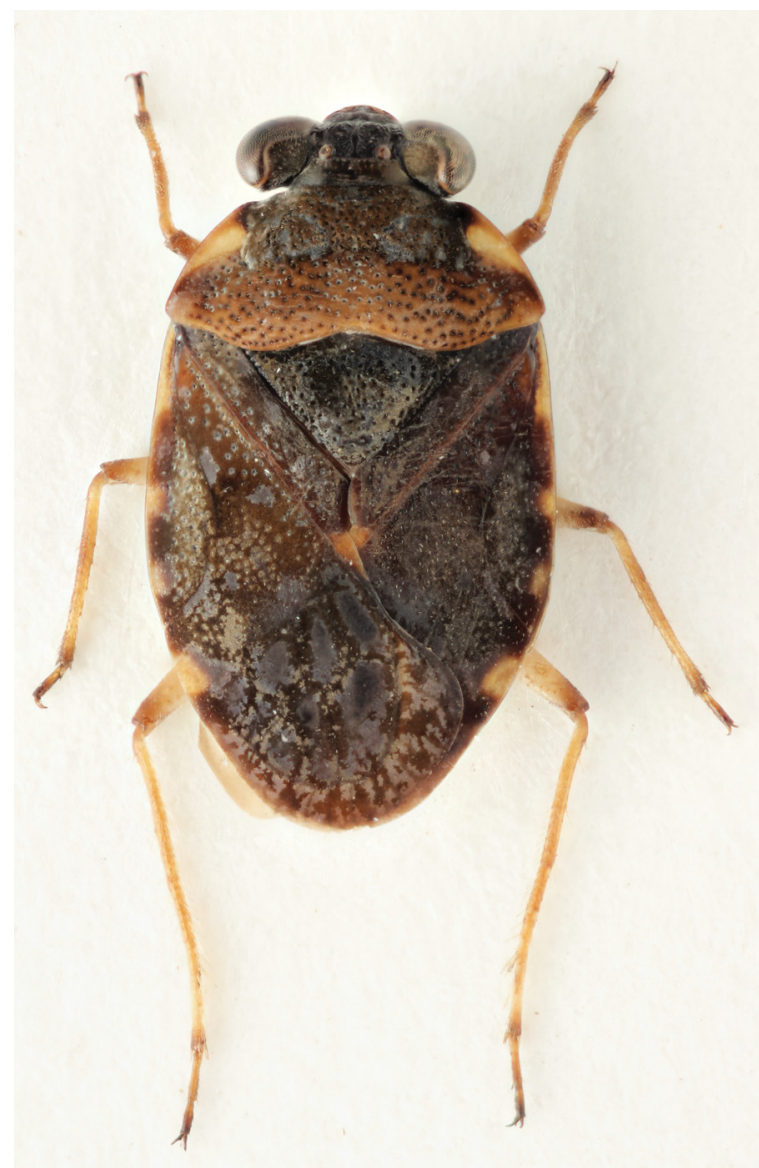

Fig. 18. Ochterus bacchusi Baehr, 1990, male, Australia: New South Wales: Ku-ring-gai Chase NP, $4.31 \mathrm{~mm}$.
Distribution. Australian Region: Australia: New South Wales (Kormilev 1971, ANDERsen \& Weir 2004), Northern Territory (BAEHr 1990b), Queensland (KormiLev 1971; Baehr 1989, 1990b; ANDERsen \& Weir 2004), South Australia (Kormilev 1971, Baehr 1989, Andersen \& Weir 2004), Tasmania (MANCINI 1939a, BAEHR 1990b, ANDERSEN \& WeIr 2004), Victoria (BAEHr 1990b, ANDERSEN \& WeIR 2004), Western Australia (KoRmilev 1971, ANDERsen \& Weir 2004); New Caledonia (Distant 1920, as O. marginatus; JACZEWSKi 1934; KoRmilev 1971; BAeHr 1990b; Andersen \& Weir 2004); Papua New Guinea (Mancini 1939a, BAEHR 1990b); Vanuatu (as New Hebrides) (JACZEWSKi 1934; BAEHR 1989, 1990b; ANDERSEN \& WeIR 2004); Solomon Islands (ANDERSEN \& Weir 2004): Choiseul (Kormilev 1971), Guadalcanal (Kormilev 1971, BAEHR 1990b), San Cristobal (Kormilev 1971).

Note. All the syntypes of $O$. australicus from Australia (JACZEWSKI 1934) belong to O. eurythorax (see BAEHR 1990b). Several of KoRMILEV's (1971) records from Victoria, southeastern Queensland, the Cape York Peninsula, Indonesia (Papua, West Papua), Papua New Guinea, and all records from Tasmania actually refer to other species (BAEHR 1989).

\section{Ochterus bacchusi Baehr, 1990}

(Fig. 18)

Ochterus bacchusi Baehr, 1990b: 452-454, 456-459, 461, 465 (key to species, description, illustrations). HoLOTYPE: $\curvearrowright$, Australia: New South Wales: 'Wentworth Falls, Blue Mts.' (BMNH).

Ochterus bacchusi: CASSIS \& GROSS (1995): 260-261 (catalogue); ANDERSEN \& WEIR (2004): 267-268, 270, 335 (key to species, illustrations, checklist).

Material examined. AUSTRALIA: New South Wales: Ku-ring-gai Chase National Park, near McCarrs Creek, 22.x.1993, 17 §ठ 15 우, P. Štys lgt., P. Kment det. (13 11 우 MMBC, 3 의 3 NMPC, $1 \delta 1+$ ZJPC).

Distribution. Australian Region: Australia: New South Wales (BAEHr 1990b, ANDERSEN \& WeIr 2004).

\section{Ochterus baehri baehri Rieger, 1977}

Ochterus baehri Rieger, 1977: 215-216 (description, differential diagnosis, illustrations). HоLотYPE: A Australia: Queensland: 'Stewart River, Cope [= Cape] York Peninsula, North Queensland' (CRNG).

Ochterus baehri baehri: BAEHR (1989): 114, 116, 118-119 (key to species, redescription, illustrations); BAEHR (1990b): 451, 453, 455-456, 472 (key to species, illustrations); CAssis \& Gross (1995): 261 (catalogue); ANDERSEN \& Weir (2004): 268, 335 (key to species, checklist).

Distribution. Australian Region: Australia: Queensland (Rieger 1977; BAeHr 1989, 1990b; ANDERsen \& Weir 2004). Note. The type depository was cited as ZSMC in error by ANDERSEN \& WeIr (2004) (Ch. Rieger, pers. comm.).

\section{Ochterus baehri riegeri Baehr, 1989}

Ochterus baehri riegeri Baehr, 1989: 114, 116, 118-119-121 (key to species, description, illustrations). HoLотуPE: $\hat{\sigma}$, Australia: Northern Territory: 'Humpty Doo, NT' (QMBA).

Ochterus baehri riegeri: BAEHR (1990b): 451, 459, 461, 472 (key to species, illustrations); CASSIS \& Gross (1995): 261 (catalogue); ANDERSEN \& WeIr (2004): 268, 270, 335 (key to species, illustrations, checklist).

Distribution. Australian Region: Australia: Northern Territoty (BAEHr 1989, 1990b; ANDERsen \& Weir 2004). 


\section{Ochterus baltazarae Gapud \& San Valentin, 1977}

Ochterus baltazarae Gapud \& San Valentin, 1977: 272, 278-283, 285-286, 288-289, 296-298 (key to species, description, illustrations, variation; partim, except paratypes of Zambales form). HoLOTYPE: ڤે, Philippines: Luzon: 'Quezon National Park, Atimonan, Quezon' (UPLB).

Ochterus baltazarae: GAPUD (1986): 32, 36-38 (key to species, redescription, illustration); GAPUD (2003): 103, 107 (taxonomy, checklist); CHEN et al. (2005): 413 (checklist).

Distribution. Oriental Region: Philippines: Luzon (GAPUD \& SAN VALENTIN 1977).

Note. Specimens of 'Zambales form' belong actually to O. luzonicus Gapud, 2003 (GAPUD 2003).

\section{Ochterus banksi Barber, 1913}

Ochterus banksi Barber, 1913: 213-214-215 (key to species, original description, illustration). Syntypes: 3 o , USA: Virginia: 'at Glencarlyn' (coll. N. Banks $\rightarrow$ AMNH).

Ochterus banksi: VAN Duzee (1917): 476 (catalogue); Hungerford (1919): 51-53 (key to species, original description repeated); BLATCHLEY (1926): 1020-1021 (key to species, redescription); SCHELL (1943a): 32, 35 (key to species, illustration); DraKe (1943b): 45 (original description repeated, differential diagnosis); Вовв (1951): 93-100 (redescription, description of egg and larval instars I-V, biology, ecology); Drake (1952): 74 (checklist); ChapMan (1958): 119-120 (key to species); WILson (1958): 154 (key to species); BoBB (1971): 17-20 (key to species, redescription, larval instars, illustrations); Polhemus \& Polhemus (1988a): 542 (catalogue); EPler (2006): 12.1-12.4 (key to species, variability, illustrations); HoffMAn (2012): 59 (illustration, distribution); Polnemus \& Polhemus (2016): 11, 13, 17, 26-30 (key to species, redescription, illustrations, new synonymy).

Ochterus banksii (incorrect subsequent spelling): HecKMAN (2011): 463 (key to species, illustration).

Ochterus flaviclavus Barber, 1913: 213, 215 (key to species, original

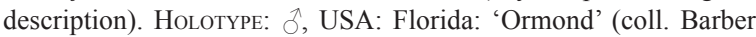
$\rightarrow$ USNM). Junior subjective synonym, suggested by EPLER (2006: 12.4), confirmed by Polhemus \& Polhemus (2016: 26).

Ochterus flaviclavus: VAN DuzeE (1917): 476 (catalogue); Hungerford (1919): 51-52 (key to species, original description repeated); BLATCHLEY (1926): 1020, 1022 (key to species, redescription); SCHELL (1943a): 32, 35 (key to species, illustration); SCHELL (1943b): 40-41 (original description repeated, differential diagnosis); DraKe (1952): 74 (checklist); ChAPMAN (1958): 119-120 (key to species); PolHEMUS \& Polhemus (1988a): 542 (catalogue); Epler (2006): 12.3-12.4 (key to species, taxonomy, illustrations); Heckman (2011): 460 (key to species, illustration)

Material examined. USA: IllinoIs: Union Co., Pine Hills 3, 10.viii.1984, J. E. McPherson, R. J. Packauskas \& T. E. Vogt lgt., Z. Jindra det., P. Kment revid. (ZJPC). New York: White Plains, 15.xii.[19]17, $1 \AA$, JRTB Collr [= J. R. Torre Bueno lgt.], C. J. Drake det. (NMPC).

Distribution. Nearctic Region: USA: Connecticut (PoLHEMus \& Polhemus 2016), Florida (Drake 1913, 1914; BlatCHLEY 1926, both as O. flaviclavus; SCHELl 1943b; DrAKE 1952; Chapman 1958; Polhemus \& Polhemus 1988a, 2016; Epler 2006), Illinois (Tinerella et al. 2009), Indiana (BLATChley 1926; Polhemus \& Polhemus 1988a), Kansas (PolheMus \& Polhemus 1988, as O. flaviclavus; 2016), Louisiana (Polmemus \& Polhemus 1988a, as O. flaviclavus; 2016), Maryland (Polhemus \& Polhemus 2016), Massachusetts (Drake 1952, Polhemus \& Polhemus 1988a), Michigan (Polnemus \& Polhemus 2016), Mississippi (Drake 1952; Wilson 1958; Polhemus \& Polhemus 1988a, 2016); New Jersey (Schell 1943b, Drake 1952, Polhemus \& Polhemus 1988a), New York (Blatchley 1926, LeONARd 1928, Schell 1943b, Drake 1952, Polhemus \& Polhemus 1988a), Ohio (Polhemus \& Polhemus 2016), Pennsylvania (Polhemus \& Polhemus 2016), South Carolina (BobB 1951, Polhemus \& Polnemus 1988a), Texas (Polhemus \& Polnemus 1988a, 2016; Polhemus 1997; Epler 2006), Virginia (BARBer 1913; Blatchley 1926; Schell 1943b; Drake 1952; Bobb 1951, 1974; Polmemus \& Polhemus 1988a, 2016; Hoffman 2012), West Virginia (Polhemus \& Polhemus 2016).

Note. BARBER (1913) described the species based on a single male from the collection of Mrs. Annie Trumbull Slosson. Later, ScHell (1943b) mentioned that the holotype was deposited in Barber's collection. Currently it is deposited in USNM (T. Henry, pers. comm.).

\section{Ochterus barberi Schell, 1943}

Ochterus barberi Schell, 1943a: 32, 35 (key to species, illustration). Lectotype (designated by Schell 1943b: 42, as holotype): đ, USA: Arizona: 'Colorado Canyon' [= Grand Canyon of Colorado River] (USNM).

Ochterus barberi: Schell (1943b): 41-42 (original description, differential diagnosis); Drake (1952): 74 (checklist); Menke (1979): 125 (distribution, illustration); Polnemus \& Polhemus (1988a): 542 (catalogue); Polhemus (1992b): 442 (note on type labels); Heckman (2011): 287, 463 (key to species, illustration); Polmemus \& PolHemus (2016): 17, 30-33 (key to species, redescription, illustrations).

Distribution. Nearctic Region: USA: Arizona (SCHELL 1943b; Drake 1952; Polnemus 1973; Polhemus \& Polhemus 1976, 1988a, 2016; Stevens \& Polhemus 2008), California (Menke 1979; Polhemus \& Polhemus 1988a, 2016), Colorado (Polhemus \& Polhemus 2016), New Mexico (Drake 1952; Polhemus 1973; Polhemus \& Polhemus 1988a, 2016); Mexico: Sonora (Polhemus \& Polhemus 1988a, 2016).

Note. Schell (1943b) also listed paratypes [actually paralectotypes] from Orizaba, in the Mexican state of Veracruz, but these specimens have proven on closer examination to represent females of $O$. aeneifrons (PolHEMus \& PolHemus 2016).

The revision of American Ochterus including the description of $O$. barberi was published divided in two parts, first published in January 1943 (Schell 1943a) and second in April 1943 (Schell 1943b). The species was first introduced in the key, which is sufficient to make it available (ICZN 1999: Art. 13.1.1), without any mention of type specimens (SCHELL 1943a: 32), while the original description including the holotype designation appeared in the second part (ScHell 1943b: 41). As a holotype is defined as 'the single specimen upon which a new nominal species-group taxon is based in the original publication' (ICZN 1999: Art. 73.1), in this case, the subsequently designated 'holotype' must be considered the lectotype according to ICZN (1999: Art. 74.5).

\section{Ochterus barrosoi Gapud, 1981}

Ochterus barrosoi Gapud, 1981: 305-307-309 (description, differential diagnosis, illustrations). Holotype: 今, Philippines: Leyte: 'Calbiga-a River, Mt Pangasugan' (UPLB).

Ochterus barrosoi: GAPUD (1981): 107 (checklist); CHEN et al. (2005): 413 (checklist).

Distribution. Oriental Region: Philippines: Leyte (GAPUD 1981). 


\section{Ochterus bidentatus Schell, 1943}

Ochterus bidentatus Schell, 1943a: 32, 35 (key to species, illustration). Lectotype (designated by Schell 1943b: 44, as holotype): §, Peru: 'Vic. San Pedro' (SEMC).

Ochterus bidentatus ScHELl (1943b): 44 (original description, differential diagnosis); Drake (1952): 74 (checklist); Heckman (2011): 462-463 (key to species, illustration).

Distribution. Neotropical Region: South America: Peru (Schell 1943b, Drake 1952, Nieser \& Chen 1992).

Note. The revision of American Ochterus including the description of $O$. bidentatus was published divided in two parts, the first published in January 1943 (SCHELL 1943a) and the second in April 1943 (Schell 1943b). The species was first introduced in the key, which is sufficient to make it available (ICZN 1999: Art. 13.1.1), without any mention of type specimens (SCHELL 1943a: 32), while the original description including the holotype designation appeared in the second part (SCHELl 1943b: 44). As a holotype is defined as 'the single specimen upon which a new nominal species-group taxon is based in the original publication' (ICZN 1999: Art. 73.1), in this case, the subsequently designated 'holotype' must be considered the lectotype according to ICZN (1999: Art. 74.5).

\section{Ochterus brachysoma Rieger, 1977}

Ochterus brachysoma Rieger, 1977: 214-215 (description, differential diagnosis, illustrations). HoLOTYPE: $\hat{\jmath}$, Australia: Queensland: 'Stewart River, Cope [= Cape] York Peninsula, North Queensland' (CRNG).

Ochterus brachysoma: BAEHR (1989): 114, 116, 118, 121-122 (key to species, redescription, illustrations); BAEHR (1990b): 450, 453, 455, $456,459,461,472-473$ (key to species, redescription, illustrations); Cassis \& Gross (1995): 261 (catalogue); Andersen \& Weir (2004): 267, 270, 335 (key to species, illustrations, checklist).

Distribution. Australian Region: Australia: Queensland (RIEGER 1977; BAeHr 1989, 1990b; ANDERSEN \& WeIr 2004).

\section{Ochterus breviculus Nieser \& Chen, 1992}

Ochterus breviculus Nieser \& Chen, 1992: 9-11 (description, differential diagnosis, illustrations). HoLotyp: 2 , China: Tibet: 'Beibeng, 850 m' (IZAS).

Ochterus breviculus: Polhemus (1995a): 25 (catalogue); XIE \& LiU (2013): 6 (checklist).

Distribution. Oriental/Palaearctic Region: China: Yunnan (NIESER \& Chen 1992, XIE \& Liu 2013), Xizang (= Tibet) (Nieser \& CHEN 1992, XIE \& LiU 2013).

\section{Ochterus bruneiensis Zettel \& Lane, 2010}

Ochterus bruneiensis Zettel \& Lane, 2010: 98-101 (description, illustrations). Ноцотүе: $\hat{\jmath}$, Brunei: Brunei-Muara District, Sungai Akar, waterfall area near main road, $47 \mathrm{~m}$ a.s.1., N 04 $56^{\prime} 45^{\prime \prime} \mathrm{E} 114^{\circ} 59^{\prime} 13^{\prime \prime}$ (BMKB)

Distribution. Oriental Region: Brunei (Zettel \& LANE 2010).

\section{Ochterus brunneus Hungerford, 1927}

Ochterus brunneus Hungerford, 1927: 188-189 (description, differential diagnosis). Syntypes: 2 đa, Bolivia: 'Huachi Rio Beni' (USNM).

Ochterus brunneus: ScHELl (1943a): 32, 35 (key to species, illustration), Schell (1943b): 41 (original description repeated, differential diagnosis); Drake (1952): 74 (checklist); HeCKMAN (2011): 461 (key to species, illustration).
Distribution. Neotropical Region: South America: Bolivia (Hungerford 1927, Drake 1952).

\section{Ochterus caffer (Stål, 1855) \\ (Figs 8, 19)}

Pelogonus caffer Stål, 1855: 46 (original description). SynTYPE(s): South Africa: Natal (NHRS)

Pelogonus marginatus (partim): STÅL (1866): 170-171 (redescription, catalogue); STÅL (1876): 137 (catalogue).

Ochterus marginatus caffer: JACZEWSKI (1934): 606-607 (differential diagnosis, illustrations); JACZEWSKI (1935): 483 (key to species); PoISSON (1948): 169 (distribution); PoIsson (1949): 48 (distribution); PoIsson (1954): 363 (distribution); BERTRAND (1962): 882 (distribution); PoIsSON (1968a): 401 (distribution); PoISSON (1968b): 34 (distribution); Poisson \& Sallier Dupin (1969): 19 (distribution).

Ochterus caffer: Mancini (1939a): 124-125 (raised to species status, variability, illustrations); KORMILEV (1973): 2-3, 5-6, 8 (redescription, illustrations), Polhemus \& Polhemus (2008): 286 (key to species for Indian Ocean).

Material examined. ?: Environs du Tsad [= environs of Lake Chad], 1937, $5 \precsim 1$, Škulina lgt., P. Kment det. (NMPC). BENIN: SE, Cove, Zou river, 16.iv.2000, $2 \hat{\jmath}$, J. Halada lgt., P. Kment det. (ZJPC). CAMEROON: NW, Tubah sbdiv., Big Babanki, N of Bamenda, 2100 m a.s.1., xii.2009, 1 ô, R. Sehnal lgt., P. Kment det. (ZJPC). DEMOCRATIC REPUBLIC OF THE CONGO: ITURI: Congo b., Ituri, 1937, 1 ¿̊, Škulina lgt., P. Kment det. (NMPC). CENTRAL AFRICAN REPUBLIC:

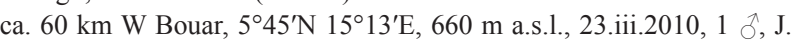
Halada lgt., P. Kment det. (MMBC); $60 \mathrm{~km} \mathrm{~W}$ Bouar, 542'N 1600'E, $860 \mathrm{~m}$ a.s.1., 30.iii.2010, 1 đ̃, J. Halada lgt., P. Kment det. (MMBC). IVORY COAST: Bouaké, 22.-23.iv.1964, 1 đ̃, R. H. Cobben lgt., P. Kment det. (ACPI). MADAGASCAR: CE, $1 \mathrm{~km}$ E of Andreba, village, 2 km E of Alaotra lake, 30.x.2003, $2 \lesssim 1$ ค, P. Baňař lgt., P. Kment det.

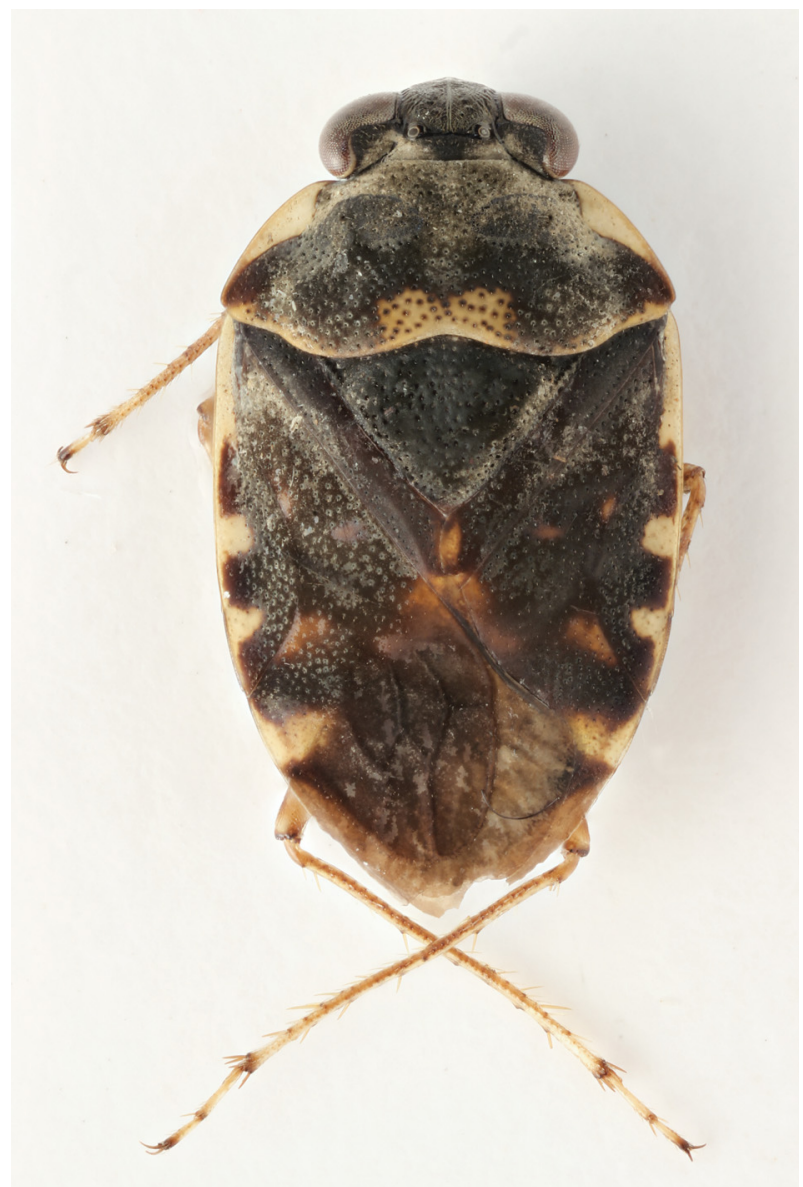

Fig. 19. Ochterus caffer (Stål, 1855), female, Sudan: Ed Damazin, $5.20 \mathrm{~mm}$. 
(1 19 MMBC, 1 NMPC); NE, Ankarafantsika N.P., 22.-24.iv.2011, ca. $100 \mathrm{~m}$ a.s.1., sweeping close to main entrance, 1 s, P. Baňař lgt., P. Kment det. (MMBC). MOZAMBIQUE: CW, 30 km NE Guro, $17^{\circ} 14^{\prime} \mathrm{S}$ $33^{\circ} 27^{\prime} \mathrm{E}, 620$ m a.s.1., 11.xii.2005, 1 1 1 , J. Halada lgt., P. Kment det. (MMBC). SOUTH SUDAN: UPPER NILE: near Malakal, 5.-20.i.1963, $1 \lesssim 1$, R. E. Linnavuori lgt., P. Kment det. (ACPI). SUDAN: BLUE Nile: Blue Nile Prov., Ed Damazin [= Ad-Damazin], 2.xii.1967, 1 § 5 $\bigcirc$ (Fig. 19), P. Štys lgt. (1169), P. Kment det. (1 4 \% MMBC, 1 NMPC). Sennar: Blue Nile prov., Dinder Game Reserve, Galegu,

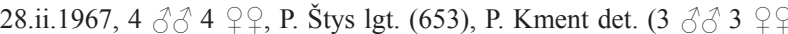
MMBC, 1 o 1 NMPC); same locality, 4.iii.1967, 4 $\delta 6$ 우, P. Štys lgt. (686), P. Štys det. as O. marginatus caffer, P. Kment revid. (3 15 $9 \mathrm{MMBC}, 1 \precsim 1$ NMPC).

Distribution. Afrotropical Region: Benin (KORMILEV 1973); Burkina Faso (Linnavuori 1981); Cameroon (Poisson 1948); Cape Verde Islands: Santiago Island (MANCini 1939a); Central African Republic (LinNAvuori 1981); Chad (Linnavuori 1981); Democratic Republic of the Congo (Poisson 1949, 1968b; Poisson \& Sallier Dupin 1969); Ethiopia (DE CARLINI 1895, MANCINI 1956); Gambia (Nieser \& Chen 1992); Guinea (Poisson 1954, Bertrand 1962); Ivory Coast (Poisson 1968a); Kenya (JACZewski 1934); Madagascar (Polhemus \& Polhemus 2008); Mozambique (new record); Nigeria (JACZEWSKI 1934, LinNAVUORI 1981); Republic of the Congo (BERTRAND 1962); São Tomé and Príncipe: Príncipe Island (MANCINI 1939a); Senegal (NIESER \& CHEN 1992); Sierra Leone (JACZEWSKI 1934); Somalia (MANCINI 1939a,b; LiNNAVUORI 1982); South Africa (STÅL 1855, JACZEWSKI 1934, KoRMILEV 1973); South Sudan (LinNAVuORI 1971, 1980); Sudan (new record); Tanzania (JACZEWSKI 1934).

\section{Ochterus cheesmannae Baehr, 1990}

Ochterus cheesmannae Baehr, 1990b: 450, 453, 455-459, 461, 473-474 (key to species, description, illustrations). Ноцотуре: Indonesia: West Papua: 'N. Dutch New Guinea: Waigeu, Camp Nok., $2500 \mathrm{ft}$ [=762 m a.s.1.]' (BMNH).

Ochterus cheesmannae: CHEN et al. (2005): 413 (checklist).

Distribution. Australian Region: Indonesia: West Papua: Waigeo Island (BAEHr 1990b, CHEN et al. 2005)

\section{Ochterus chiapensis}

\section{A. Polhemus \& J. T. Polhemus, 2016}

Ochterus chiapensis D. A. Polhemus \& J. T. Polhemus, 2016: 14, 17, 33-36 (key to species, description, illustrations). Holotype: Mexico: 'Chiapas, seeping roadside rock faces near Ixhuatan [2.5 mi. S of Ixhuatan, vic. $17^{\circ} 16^{\circ} 04^{\prime \prime N}, 93^{\circ} 01^{\prime} 26^{\prime \prime} \mathrm{W}$, elev. $\sim 640 \mathrm{~m}$ ]' $(\mathrm{JTPC} \rightarrow \mathrm{USNM})$.

Distribution. Neotropical Region: Central America: Mexico: Chiapas (Polhemus \& Polhemus 2016).

\section{Ochterus costaricensis}

\section{A. Polhemus \& J. T. Polhemus, 2016}

Ochterus costaricensis D. A. Polhemus \& J. T. Polhemus, 2016: 17, 36-39 (key to species, description, illustrations). Holotype: 3 , Costa Rica: 'Puntarenas, roadside waterfall $15 \mathrm{mi}$. N of Palmar Norte [8॰58'14" $\mathrm{N}$, $83^{\circ} 18^{`} 15^{\prime \prime} \mathrm{W}$, elev. $70 \mathrm{~m}$ ]' (JTPC $\rightarrow$ USNM).

Distribution. Neotropical Region: Central America: Costa Rica (Polhemus \& Polhemus 2016); El Salvador (Polhemus \& Polhemus 2016); Panama (Polhemus \& Polhemus 2016).

\section{Ochterus dufourii (Montrouzier, 1864)}

Pelogonus Dufourii Montrouzier, 1864: 241 (original description). Lectotype (designated by BAeHr 1990b: 456): đa, New Caledonia: 'N. Calédonie, Kanala' (ISNB).

Pelogonus Dufourii: STÅL (1876): 137 (catalogue).

Pelogonus Dufouri (incorrect subsequent spelling): ScHouteden (1907): 117 (types).

Ochterus dufourii: JACZEWSKI (1934): 609-610 (repeating original description, differential diagnosis); Kormilev (1971): 442-443 (repeating original description); BAEHR (1990b): 451, 453-454, 456-459, 461 (key to species, redescription, illustrations, lectotype designation); DAMgAard \& ZetTel (2014): 237 (checklist).

Distribution. Australian Region: New Caledonia (MonTROUZIER 1864, BAEHR 1990b).

\section{Ochterus eurythorax Baehr, 1989}

Ochterus marginatus (misidentification): HaLE (1925): 14-15 (distribution, partim) (see JACZEWSKI 1934: 607, as O. australicus).

Ochterus australicus (misidentification): JACZEWSKI (1934): 607-610 (partim, few syntypes from Australia) (see BAEHR 1990: 452).

Ochterus australicus (misidentification): KORMILEV (1971): 434: Fig. 12, 439-440, 442: Fig. 22 (redescription) (see BAEHR 1989: 115, 1990: 452).

Ochterus eurythorax Baehr, 1989: 114, 116-118 (key to species, description, illustrations). Holotype: ô, Australia: Queensland: 'Dunwich, Stradbroke Isl., S. E. Qld' (QMBA).

Ochterus eurythorax: BAEHR (1990b): 452-454, 456, 459, 461-462, 464 (key to species, redescription, illustrations); CAssis \& Gross (1995): 261 (catalogue); ANDERSEN \& WeIR (2004): 267-268, 335 (key to species, illustration, checklist).

Distribution. Australian Region: Australia: Australian Capital Territory (BAEHR 1990b, ANDERSEN \& WeIr 2004), New South Wales (JACZEWSKI 1934, as O. australicus; BAEHR 1990b; ANDERSEN \& WeIR 2004), Queensland (JACZEWSKI 1934, as O. australicus; BAEHR 1989, 1990b; ANDERSEN \& WeIr 2004), South Australia (BAEHR 1989, ANDERSEN \& WeIR 2004), Tasmania (BAeHr 1989, 1990b; ANDERSEN \& WeIR 2004), Victoria (BAEHR 1989, 1990b; ANDERSEN \& WEIR 2004). Note. Records from Papua New Guinea (BAEHR 1989) refer presumably to another species (see BAEHR 1990b).

\section{Ochterus explanatus}

\section{A. Polhemus \& J. T. Polhemus, 2016}

Ochterus explanatus D. A. Polhemus \& J. T. Polhemus, 2016: 11, 16,39-41 (key to species, description, illustrations). HоLотуPE: + , Mexico: Durango: 'Durango [incorrectly indicated as Sonora], $7 \mathrm{mi}$. W of Los Bancos [vic. $23^{\circ} 38^{\prime} 54^{\prime} \mathrm{N}, 105^{\circ} 47^{\prime} 56^{\prime \prime} \mathrm{W}$, elev. $\sim 2320 \mathrm{~m}$ ]' (JTPC $\rightarrow$ USNM).

Distribution. Neotropical Region: Central America: Mexico: Durango (Polhemus \& Polnemus 2016), Oaxaca (Polhemus \& Polhemus 2016).

\section{Ochterus feae Mancini, 1939 (Figs 20-23)}

Pelogonus marginatus (misidentification): MONTANDON (1897): 365 (variability, distribution); Distant (1906): 14 (illustration of the specimen from MonTANDON 1897).

Ochterus Feae Mancini, 1939a: 125-126 (description, differential diagnosis, illustrations). НоLотуре: $q$ (Figs 20-23), Myanmar: 'Carin: Asciuii Ghecù' (MCSN).

Ochterus feae: KormiLEv (1971): 443 (list).

Material examined. LAOS: Luang Prabang: Louang Pharabang prov., Ban Song Cha (5 km W), 20 $33-34^{\prime} \mathrm{N} 102^{\circ} 14^{\prime} \mathrm{E}$, ca. $1200 \mathrm{~m}$ a.s.l.,

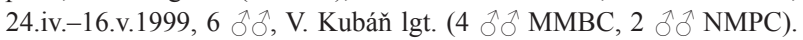
Oudomxay: Oudomxai (17 km NEE), 2046' $\mathrm{N} 102^{\circ} 09^{\prime} \mathrm{E}$, ca. $1100 \mathrm{~m}$ 

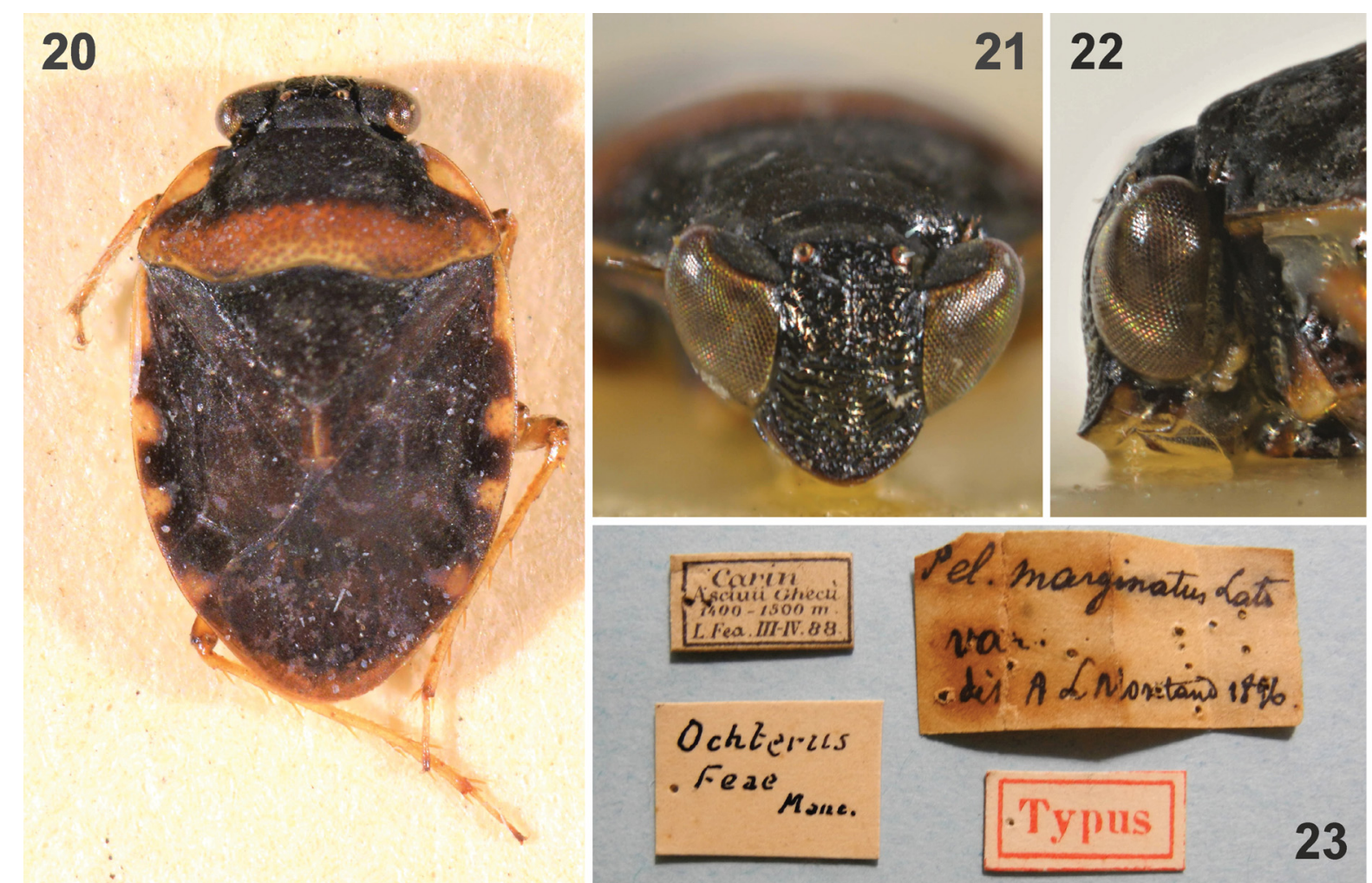

Figs 20-23. Ochterus feae Mancini, 1939, female, holotype: 20 - habitus in dorsal view; 21 - head, frontal view; 22 - head, lateral view; 23 - type labels. Photographs by R. Poggi.

a.s.1., 1.-9.v.2002, 1 1 1 , V. Kubán̆ lgt. (MMBC). MYANMAR: Chin: Chin hills, NW of Falam, 400--500 m a.s.1., v.--vi.2016, 12 o 6 \% 9 , A. Carapezza det. (ACPI). THAILAND: ChiAng MaI: San Pakia village, $19.19^{\circ} \mathrm{N} 98.50^{\circ} \mathrm{E}, 1400 \mathrm{~m}$ a.s.1., 1 đ3 3 우, 1.-15.v.1998, V. Kubáň lgt. (1 2 + 9 MMBC, 1 \% NMPC). MAE Hong Son: Ban Huai Po, 1600-2000 ma.s.l., 1 ^, J. Horák lgt. (NMPC). TAK: Umphang, $16^{\circ} 04^{\prime} \mathrm{N} 98^{\circ} 53^{\prime} \mathrm{E}, 500$ m a.s.1., 26.iv.-6.v.[19]91, 1 +, D. Král lgt. (NMPC). All P. Kment det.

Distribution. Oriental Region: Laos (new record); Myanmar (MonTANDon 1897, Distant 1906, as P. marginatus; MANCINI 1939a); Thailand (new record).

Note. This species was described based on a female holotype and has never been recorded again. It is related to $O$. marginatus, from which it differs mainly by the anterior margin of the head being distinctly reflexed (see KORMILEV 1971). The photographs of the holotype of O. feae (Figs 20-23) are presented here for the first time.

\section{Ochterus foersteri Kormilev \& De Carlo, 1952}

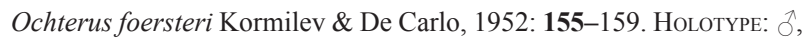
Paraguay: 'Caaguazú, Paso Yobai' (MACN).

Ochterus foersteri: KoRMILEV \& DeCARLo (2017): 204 (catalogue); BACHMANN (1999): 207 (types); HeCKMAN (2011): 461 (key to species).

Distribution. Neotropical Region: Argentina: Misiones (Melo 2015; Bachmann \& Mazzucconi 1995, 2017; Bachmann 1998); Paraguay (Kormilev \& De Carlo 1952, Bachmann \& Mazzucconi 2017).

\section{Ochterus grandiusculus Nieser \& Chen, 1992}

Ochterus grandiusculus Nieser \& Chen, 1992: 11-12 (description, differential diagnosis, illustration). НоLотуре: $\delta$, Indonesia: Sulawesi: 'Sulawesi Tenggara, road Kolaka-Kendari Km 20' (MUDH).

Ochterus grandiusculus: Nieser \& CHEN (1999): 89-90 (key, illustration); CHEN et al. (2005): 413 (checklist).
Distribution. Australian Region: Indonesia: Sulawesi (Nieser \& Chen 1992).

\section{Ochterus gressitti Kormilev, 1971}

Ochterus gressitti Kormilev, 1971: 434, 438-439 (description, differential diagnosis, illustrations). HoLotyPE: 0 , Papua New Guinea: Morobe Province: 'NE New Guinea, Wampit V. near Gurakor village, near Wau, 550 m' (BPBM).

Ochterus gressitti: GAPUD (1981): 303-305, 307 (diagnosis, illustrations); BAEHR (1990b): 451, 453, 455-459, 461, 471 (key to species, redescription, illustrations); CHEN et al. (2005): 413 (checklist).

Distribution. Australian Region: Indonesia: Papua (KoRMilev 1971), West Papua (Kormilev 1971); Papua New Guinea (Kormilev 1971, Gapud 1981, Baehr 1990b), incl. New Britain (KoRmilev 1972).

\section{Ochterus homorfos Nieser \& Chen, 1999}

Ochterus homorfos Nieser \& Chen, 1999: 90-91-92, 123 (key, description, illustrations). Ноцотуре: ${ }^{2}$, Indonesia: Sulawesi: 'Sulawesi Utara, Gunung Ambang NSG' (NHMW).

Ochterus homorfos: CHEN et al. (2005): 413 (checklist).

Distribution. Australian Region: Indonesia: Sulawesi (Nieser \& Chen 1999).

\section{Ochterus hungerfordi Schell, 1943}

Ochterus americanus (partim, misidentification): UHLER (1876): 336 (specimen from Cuba).

Ochterus hungerfordi Schell, 1943a: 32, 35 (key to species, illustration). Lectotype (designated by Schell 1943b: 37, as holotype): ¿̂, Cuba: 'Mantanzas, Mumuri Valley' (SEMC).

Ochterus hungerfordi: ScHELL (1943b): 37 (original description, differential diagnosis); DraKe (1952): 74 (checklist); Alayo (1971): 4-5 (key to species); Alayo (1974): 34-35 (key to species); Heckman (2011): 459 (key to species, illustration). 
Material examined. CUBA: Sierra Maestra, x.[19]28, 1 으. Dr. Rambousek lgt., L. Hoberlandt det., P. Kment revid. (NMPC).

Distribution. Neotropical Region: Caribbean Islands: Cuba (Schell 1943b; Drake 1952; Alayo 1971, 1974; MuÑoz Riviaux et al. 2010a,b; NARAnJo et al. 2010).

Note. The revision of American Ochterus including the description of $O$. hungerford $i$ was published divided in two parts, the first published in January 1943 (SCHELL 1943a) and the second in April 1943 (Schell 1943b). The species was first introduced in the key, which is sufficient to make it available (ICZN 1999: Art. 13.1.1), without any mention of type specimens (SCHELL 1943a: 32), while the original description including the holotype designation appeared in the second part (Schell 1943b: 37). As a holotype is defined as 'the single specimen upon which a new nominal species-group taxon is based in the original publication' (ICZN 1999: Art. 73.1), in this case, the subsequently designated 'holotype' must be considered the lectotype according to ICZN (1999: Art. 74.5).

\section{Ochterus jaczewskii Kormilev, 1971}

Ochterus jaczewskii Kormilev, 1971: 434, 439 (description, differential diagnosis, illustrations). HоLотүPE: 3 , Indonesia: West Papua: 'NW New Guinea, Vogelkop, Irai R., N of Lake Anggi Giji, 1850 m’ (BPBM).

Ochterus jaczewski (incorrect subsequent spelling): GAPUD (1981): 302-303, 305, 307 (diagnosis, illustrations).

Ochterus jaczewskii: BAEHR (1990b): 452-454, 456-459, 461, 466-467 (key to species, redescription, illustrations); CHEN et al. (2005): 413 (checklist).

Distribution. Australian Region: Indonesia: Papua (Polhemus \& Polhemus 1998), West Papua (Kormilev 1971, BAEHR 1990b); Papua New Guinea, incl. Normanby Island (KoRMiLEv 1971, 1972; GAPUd 1981).

\section{Ochterus kokodae Baehr, 1990}

Ochterus kokodae Baehr, 1990b: 452-454, 456-459, 461, 465-466 (key to species, description, illustrations). Holotype: $\hat{\jmath}$, Papua New Guinea: 'Papua, Kokoda, $1200 \mathrm{ft}$. [= $366 \mathrm{~m}$ a.s.1.]' (BMNH).

Ochterus kokodae: CHEN et al. (2005): 413 (checklist).

Distribution. Australian Region: Papua New Guinea (BAEHr 1990b, CHEN et al. 2005).

\section{Ochterus latior Baehr, 1990}

Ochterus latior Baehr, 1990b: 451, 453, 455-458, 474-475 (key to species, description, illustrations). Holotype: + , Papua New Guinea: 'Madang Distr., Finisterre Mts., Momo C., 5550 ft. [= $1692 \mathrm{~m}$ a.s.1.]' (BMNH).

Ochterus latior: CHEN et al. (2005): 413 (checklist).

Distribution. Australian Region: Papua New Guinea (BAeHr 1990b, Chen et al. 2005).

\section{Ochterus louisiadae Baehr, 1990}

Ochterus louisiadae Baehr, 1990b: 451, 453, 455-459, 461, 470-471 (key to species, description, illustrations). Hоцотүр: $\hat{\jmath}$, Papua New Guinea: Rossel Island: 'SE: Louisiade Archipelago, Yela I., Wo Po River, 400 m' (BMNH).

Ochterus louisiadae: CHEN et al. (2005): 413 (checklist).

Distribution. Australian Region: Papua New Guinea: Louisiade Archipelago (BAEHR 1990b, CHEN et al. 2005).

\section{Ochterus luzonicus Gapud, 2003}

Ochterus baltazarae (partim, paratypes of Zambales form): GAPUD \& SAN VALENTIN (1977): 280, 282-283, 296-298 (diagnosis, illustrations). Ochterus luzonicus Gapud, 2003: 100-103, 107. HoLotyPe: Philippines: Luzon: 'La Union: Bacnotan, Don Mariano Marcos Memorial State University' (VPGP).

Ochterus luzonicus: CHEN et al. (2005): 413 (checklist).

Distribution. Oriental Region: Philippines: Luzon (GAPUD 2003).

\section{Ochterus magnificus}

Gapud \& San Valentin, 1977

Ochterus magnificus Gapud \& San Valentin, 1977: 272, 282-283-289, 294, 296-298 (key to species, description, illustrations). Ноцотуре: $\widehat{\jmath}$, Philippines: Luzon: ‘National Botanic Garden, Real, Quezon'(UPLB).

Ochterus magnificus: GAPUD (1986): 32, 38-40 (key to species, redescription, illustration); GAPUD (2003): 107 (checklist); CHEN et al. (2005): 413 (checklist)

Distribution. Oriental Region: Philippines: Luzon (GAPUD \& SAN VALENTIN 1977).

\section{Ochterus magnus Gapud \& San Valentin, 1977}

Ochterus magnus Gapud \& San Valentin, 1977: 272, 282, 285-287-291, 296-298 (key to species, description, illustrations). Ноцотуре: $\partial$, Philippines: Luzon: 'Humayao Creek, Pajo, Alfonso, Cavite'(UPLB). Ochterus magnus: GAPUD (1986): 32, 40-42 (key to species, redescription, illustration); GAPUD (2003): 107 (checklist); CHEN et al. (2005): 413 (checklist)

Distribution. Oriental Region: Philippines: Luzon (GAPUD \& SAN Valentin 1977, Gapud 1981).

\section{Ochterus manni Hungerford, 1927}

Ochterus manni Hungerford, 1927: 189 (description, differential diagno-

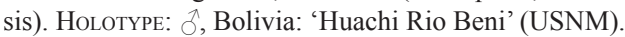

Ochterus manni: Schell (1943a): 32, 35 (key to species, illustration); SCHELl (1943b): 46 (original description repeated, differential diagnosis); Drake (1952): 74 (checklist); Heckman (2011): 463 (key to species, illustration); CiANFERONI (2012): 67 (key to species); HERRERA MAdRigal (2015): 256 (illustrations); Polhemus \& PolHemus (2016): 12, 15-16, 41-44 (key to species, redescription, illustrations).

Material examined. PANAMA: DARIÉn: Cerro Chucantí Reserve, base camp, $8^{\circ} 47.3^{\prime} \mathrm{N} 78^{\circ} 27.1^{\prime} \mathrm{W}, 800-1000 \mathrm{~m}$ a.s.l., lower montane forest, at light, 27.-30.viii.2017, 2 + 9 , M. Fikáček, J. Hájek, M. Seidel \& L. Sekerka lgt., P. Kment det. (NMPC).

Distribution. Neotropical Region: Central America: Costa Rica (Schell 1943b, Herrera Madrigal 2015, Polhemus \& Polhemus 2016), Panama (Polhemus \& Polhemus 2016); Bolivia (Hungerford 1927, Drake 1952, Polhemus \& Polhemus 2016). South America: Brazil: Pará (Polhemus \& Polhemus 2016), Ecuador (Cianferoni 2012, Polhemus \& Polhemus 2016), Peru (Schell 1943b, Polhemus \& Polhemus 2016). Note. According to Polhemus \& Polhemus (2016), O. unidentatus Nieser \& Chen, 1992 described from Ecuador may be synonym of this species.

\section{Ochterus marginatus marginatus (Latreille, 1804) (Fig. 7)}

Acanthia marginata Latreille, 1804: 242-243 (original description). Syntype(s): France: Bordeaux (depository unknown, cf. Polhemus 1995a: 25).

Ochterus marginatus: LATREILlE (1807): 143 (new combination). Pelogonus marginatus Latreille, 1809: 384 (new combination). 
Pelogonus marginatus: Le Peletier \& Serville (1825): 33 (redescription); Dufour (1833): 192-196, Pl. V: fig. 58 (diagnosis, distribution, illustration); BURMEISTER (1835): 203-204 (diagnosis, distribution); BRULlÉ (1836): 277-278 (redescription, distribution); BLANCHARD (1840): 93-94 (redescription, catalogue); АMYot \& SERville (1843): 409 (diagnosis, distribution); Herrich-SCHÄFFER (1849): 18, 23-24, Pl. CCXC: fig. 892 (diagnosis, catalogue, illustration); FIEBER (1861): 103 (catalogue); Costa (1862): 331 (redescription); REUTER (1888): 721-722 (catalogue); Oshanin (1909): 955-956 (catalogue); SinGH-Pruthi (1925): 184, Pl. XVIII: fig. 130 (male genitalia, illustrations).

Pelogonus marginatus (partim): STÅL (1866): 170-171 (redescription, catalogue); StÅl (1876): 137 (catalogue); Distant (1906): 14 (redescription).

Pelegonus marginatus: Puton (1869): 38 (list); Puton (1880): 209 (redescription, distribution).

Ochterus marginatus: HOFFMANN (1933): 249-250 (catalogue); JACZEWSKI (1935): 482 (key to species); Wu (1935): 557 (catalogue); Kormilev (1971): 433-436 (redescription, illustrations, variation); KoRMILEV (1973): 2-4, 8 (diagnosis, variability, illustrations); RIEGER (1976): 109-188 (head and prothorax morphology, illustrations); RIEGER (1977): 213-214 (differential diagnosis, illustration); TAMANINI (1979): 18, 45, 51 (key, redescription, illustrations); NIESER (1982): 11, 46 (illustration, distribution); Boulard \& COFFIN (1991): 58-67 (adult and larval morphology, biology); NIESER \& CHEN (1992): 9 (illustration of paramere); NIESER et al. (1994): 4, 20-21, 44 (key, illustrations); CHEN (1999): 9-10 (diagnosis, illustrations); NIESER \& CHEN (1999): 90-91 (key, illustration); CHEN et al. (2005): 78-79, 81,413 (illustrations, bionomics, checklist); KanYukova (2006): 46-47 (redescription, illustrations); Polhemus \& Polhemus (2008): 286 (key to species); Linnavuori et al. (2011): 76 (photo); PolHemus \& Polnemus (2012): 345 (key to species); Polnemus \& Polhemus (2015): 207, 209-210 (key to species, illustrations); Popov \& HeIss (2014a): 187-188 (illustrations).

Ochterus marginatus marginatus: JACZEWSKI (1934): 601-605 (redescription, variability, illustrations); JACZEWSKI (1935): 483 (key to species); POISSON (1957): 24-25 (redescription, illustrations); HofFMANN (1941): 67-69 (catalogue); Polhemus (1995a): 25 (catalogue); Polhemus \& Polhemus (2012): 345-347 (diagnosis, variability, illustrations); Aurema et al. (2013): 8 (catalogue); XIE \& LiU (2013): 6-7 (checklist); Thirumalai (2017): 15-16, 69 (catalogue); НAyashi \& МiYamoto (2018): 259, 261 (identification, illustration).

Pelogonus indicus Guérin-Méneville, 1843: 113 (original description, differential diagnosis). SyNTYPE(s): India: 'le plateau des Neelgherries, dans le Coromandel' [= Nilgiri Hills] (MZFN, see Polhemus 1995a: 25). Junior subjective synonym by MonTANDON (1910: 1).

Pelogonus indicus: STÅl (1876): 137 (catalogue).

Pelogonus armeniacus Kolenati, 1857: 454-455. Syntypes: 6 ( $\left.\delta^{\circ}\right)$, Azerbaijan: Karabagh: 'in littore lacuum alpestrium [= at coast of alpine lakes] Balloch-ghöll, Sullü-ghöll, Schamlogh-ghöll, Kaepesghöll in provincia Karabagh' (ZMAS, probably additional syntypes in NHMW, see PoLHEMus 1995a: 25). Junior subjective synonym by Kiritshenko (1918: 172).

Pelogonus armeniacus: STÅL (1876): 137 (catalogue).

Pelogonus flavomarginatus Scott, 1874: 446-447 (original description). Holotype: ?sex, Japan (BMNH). Junior subjective synonym by JACZEWSKI (1934: 598, 605).

Pelogonus flavo-marginatus (incorrect subsequent spelling): STÅL (1876): 137 (catalogue).

Pelogonus flavomarginatus: MATSUMURA (1915): 103 (catalogue).

Ochterus marginatus flavomarginatus: Мгчамото (1961): 230-231, 257, pl. 42: figs 235-237 (morphology of alimentary organs, illustrations); Miyamoto \& Lee (1966): 398 (catalogue, distribution); Tомокuni (1981): 111 (distribution).

Ochterus flavomarginatus: HAYASHI (1995): 20 (distribution, habitat).

Salda rutherfordi Distant, 1915: 506 (original description). HoLOTYPE: ?sex, Sri Lanka: 'Peradeniya' (BMNH). Junior subjective synonym; suspected synonymy suggested by W. E. China in JACZEWSKI (1934: 598) (see also Polhemus 1979: 112).

Pelogonus formosanus Matsumura, 1915: 103-104 (differential diagnosis). Syntypes: $2 \precsim 1$, Taiwan: 'Kagi' [= Chiayi] and 'Koshun' [= Hengchun] (EIHU). Junior subjective synonymy by EsAKI (1926:
184); upgraded to subspecies by Мгуамото (1960: 78), synonymy confirmed by Kormilev (1971: 433).

Ochterus formosanus: TAKAHASHI (1921): 119-125 (biology); TAKAHASHI (1923): 67-68 (biology).

Ochterus thienemanni (misidentification): NIESER \& CHEN (1992): 12 (corrected by NiESER \& CHEN 1999).

Material examined. Palaearctic Region: Europe: BULGARIA: K'rdzhali env., Arda river, 23.viii.1948, $4 \lesssim 1$ ๆ , M. Josifov det. (NMPC); Momchilgrad, Djebel, 24.vi.1961, 6 3 30 , L. Hobrlandt \& Slouková lgt., P. Kment det. (NMPC). FRANCE: Dép. du Var, [18]99, 1 \%, no collector, L. Hoberlandt det. (NMPC); Dept. Var, Le Lavandou, 1930, 1 స, J. Obenberger lgt., P. Kment det. (NMPC); Mandelieu, Esleret [?], viii., 1 , , Bordeau lgt., J. Roubal det., P. Kment revid. (MMBC). GREECE: East Macedonia And Thrace: Drama, Paranestion, 15.v.1937, 1

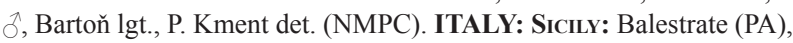
7.vii.1972, 1 larva, 22.xi.1972, 1 larva, A. Carapezza lgt. \& det. (ACPI); Madonie, Petralia Sottana, 1000 m a.s.1., 9.vi.1972, 1 \%, A. Carapezza lgt. \& det. (ACPI); Mezzoiuso (PA), 22.v.1977, 1 ô, A. Carapezza lgt. $\&$ det. (ACPI). Palermo Prov., Scillato env., Torrente Fichera, ca. $180 \mathrm{~m}$ a.s.1., 28.iv.2014, 1 § , J. Hájek \& K. Kalíková lgt., P. Kment det. (NMPC). North Africa: ALGERIA: Ghardimaou, 19.-26.iv.[19]27, 2 ○o , Mařan lgt., P. Kment det. (NMPC). Asia: AFGHANISTAN: LaGhman: Nengrahar prov., Laghman (30), 860 m a.s.1., 20.iii.1966, 1 ふै, Povolný \& Tenora lgt., P. Kment det. (MMBC). Nuristan: Bashgultal [= Landai Sin valley], $1100 \mathrm{~m}$ a.s.1., 14.vii.1952, J. Klapperich lgt., P. Kment det. (NMPC). CHINA: ANHUi: Dabieshan Mts., 65 km SW of Huoshan, 1400 m a.s.1., 23.vi.1998, 1 ₹ 1 †, L. Bocák lgt., P. Kment det. (ZJPC). BEIJING: Pearl Lake [= Zhenzhu Lake], $70 \mathrm{~km} \mathrm{~W}$ of Beijing, 16.-18.vi.2000, 1 , Z. Jindra lgt., P. Kment det. (ZJPC). FuJIAN: Kuatun, 9.vii.1946, 1 , Tschung Sen lgt., P. Kment det. (NMPC). ShaAnXı: Qing Ling Shan Mts., road Baoji-Taibai village, ca. $35 \mathrm{~km} \mathrm{~S}$ of Baoji, 18.vii.1998, 1 ,, $\mathrm{Z}$. Jindra lgt., P. Kment det. (ZJPC). Yunnan: Cheli env., $700 \mathrm{~m}$ a.s.l., 30.iv.1957, 1 L5, D. Panfilov lgt. (NMPC); Lunan env., Stone Forest, 29.vii.1995, 1 స. Z. Jindra lgt., P. Kment det. (ZJPC); $50 \mathrm{~km} \mathrm{SW}$ of Mojiang, $1100 \mathrm{~m}$ a.s.1., 2.iv.1955, Kryzhanovskiy lgt., P. Kment det. (NMPC); Xiaomengyang, $810 \mathrm{~m}$ a.s.1., 26.iii.1957, $2 \lesssim 1$ ○, S.-Y. Wang lgt., P. Kment det. (NMPC); Xiaomengyang, $850 \mathrm{~m}$ a.s.1., 29.iii.1957, 2 IJ 1 , L.-C. Zang lgt., P. Kment det. (NMPC); Tengchong, S margin of the city, $24^{\circ} 59.4^{\prime} \mathrm{N} 98^{\circ} 28.9^{\prime} \mathrm{E}, 1650 \mathrm{~m}$ a.s.l., [Ch12], individually collected under stones, on soil surface, on plants and shrubs, in pool with clay bottom, margin of mixed forest above the rice fields, 1 , J. Hájek \& J. Růžička lgt., P. Kment det. (NMPC); Gengma, 5.v.1955, 1 ๆ, T.-R. Huang lgt., P. Kment det. (NMPC); 30 km SW of Jinping, Měnglā, 400 m a.s.1., 3.v.1956, 1 q, K.-R. Huang lgt., P. Kment det. (NMPC); Jingdong, $1200 \mathrm{~m}$ a.s.1., 21.iii.1957, 1 §ै, A. Monchadskiy lgt., P. Kment det. (NMPC). IRAN: Iran, no more details, 6.ix.1961, 28 o 18 우, J. Klapperich lgt., P. Kment det. (NMPC). Alborz: Kandavan - pass, S slope, 2700-2900 m a.s.1., (36 $10^{\circ} \mathrm{N} 51^{\circ} 15^{\prime} \mathrm{E}$, Loc. No. 395), 4.-9. vii.1977, 1 +, Exp. Nat. Mus. Praha lgt., P. Kment det. (NMPC); Karaj, 1200 m a.s.1., 23.vi.1960, 3 ô, J. Klapperich lgt., P. Kment det. (NMPC); Karaj, $3 \mathrm{~km} \mathrm{~N}$ of Dam, $1650 \mathrm{~m}$ a.s.1. $\left(35^{\circ} 48^{\prime} \mathrm{N} 50^{\circ} 59^{\prime} \mathrm{E}\right.$, Loc. No. 85), 10.viii.1970, $2 \delta$, Exp. Nat. Mus. Praha lgt., P. Kment det. (NMPC). ARDABIL: $5 \mathrm{~km}$ NW Kolur, 'Shahrud' river valley, $37^{\circ} 26.1^{\prime} \mathrm{N} 48^{\circ} 41.2^{\prime} \mathrm{E}$, 1670 m a.s.1., 4.-5.vi.2006, 1 9, J. Hájek \& P. Chvojka lgt., P. Kment det. (NMPC). FARs: $5 \mathrm{~km}$ E of Furk, on the road Hajiabad-Darab, 900 m a.s.1. $\left(28^{\circ} 18^{\prime} \mathrm{N} 55^{\circ} 16^{\prime}\right.$ E, Loc. No. 217), 28.v.1973, 1 ㅇ, Exp. Nat. Mus. Praha lgt., P. Kment det. (NMPC); Aliabad, 75 km NW Jahrom (29³0’N 52॰35'E; Loc. No. 53), 10.vii.1970, 1 đ 1 L, Exp. Nat. Mus. Praha lgt., P. Kment det. (NMPC); Dasht-e Arzhan, $50 \mathrm{~km} \mathrm{~W} \mathrm{of} \mathrm{Shiraz,} \mathrm{Shur} \mathrm{river}$

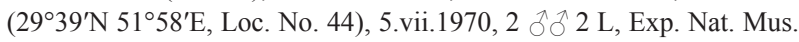
Praha lgt., P. Kment det. (NMPC); Tang-e Chogan-e Olia, ruins of Bishapur, $1000 \mathrm{~m}$ a.s.1. $\left(29^{\circ} 47^{\prime} \mathrm{N} 51^{\circ} 36^{\prime} \mathrm{E}\right.$, Loc. No. 233), 10.vi.1973, 4 $\lesssim \partial$ 1 L, Exp. Nat. Mus. Praha lgt., P. Kment det. (NMPC). GiLAn: Racht [= Rasht], 7.v.1965, 1 1 , Safavi lgt., P. Kment det. (NMPC). HormozGAN: $20 \mathrm{~km} \mathrm{SE}$ of Minab $\left(27^{\circ} 05^{\prime} \mathrm{N} 57^{\circ} 14^{\prime} \mathrm{E}\right.$, Loc. No. 204), 21.v.1973, 1 , Exp. Nat. Mus. Praha lgt., P. Kment det. (NMPC); Bashagerd [mts.], Senderk, 11.v.1977, 1 †, Safavi \& Pazuki lgt., P. Kment det. (NMPC); Faryab, Rud-e Rudan river, 350 m a.s.1. $\left(27^{\circ} 28^{\prime} \mathrm{N} 57^{\circ} 07^{\prime} \mathrm{E}\right.$, Loc. No. 201), 17.-18.v.1973, 1 을. Exp. Nat. Mus. Praha lgt., P. Kment det. (NMPC); Teleng, $40 \mathrm{~km}$ SE of Minab (26 $5^{\circ} 3^{\prime} \mathrm{N} 57^{\circ} 16^{\prime} \mathrm{E}$, Loc. No. 205), 21.v.1973, 
1292 L, Exp. Nat. Mus. Praha lgt., P. Kment det. (NMPC). Kerman: $33 \mathrm{~km}$ W Sabzvaran [= Jiroft] $\left(28^{\circ} 44^{\prime} \mathrm{N} 57^{\circ} 28^{\prime} \mathrm{E}\right.$, Locality No. 189), 1100 m a.s.1., 6.-7.v.1973, 1 9, Exp. Nat. Mus. Praha, P. Kment det. (NMPC); Deh Bakri $\left(29^{\circ} 03^{\prime} \mathrm{N} 57^{\circ} 56^{\prime} \mathrm{E}\right.$, Locality No. 186$), 1700-1750 \mathrm{~m}$ a.s.l., 30.iv.-3.v.1973, 1 1 1 spec., Exp. Nat. Mus. Praha lgt., P. Kment det. (NMPC). QAzvin: Hasan Sabbah Castle, 36²7.0'N 50³4.9'E, 2075 m a.s.l., streamlet valley, 13.v.2008, 1 \& , J. Hájek \& P. Chvojka lgt., P. Kment det. (NMPC). Sistan AND Baluchistan: 16 km SE of Tangé-Sarhé, $61 \mathrm{~km}$ NNW Nikshahr, $900 \mathrm{~m}$ a.s.1. $\left(26^{\circ} 29^{\prime} \mathrm{N} 60^{\circ} 02^{\prime} \mathrm{E}\right.$, Loc. No. 154), 10.iv.1973, 3 9 , Exp. Nat. Mus. Praha lgt., P. Kment det. (NMPC); Taftan [Mts.], Tamandan Valley $\left(28^{\circ} 36^{\prime} \mathrm{N} 61^{\circ} 02^{\prime} \mathrm{E}\right.$, Locality No. 167), 2100 m a.s.1., 20.iv.1973, 1 9, Exp. Nat. Mus. Praha lgt., P. Kment det. (NMPC). Tehran: Elburgsgeb. [= Elburz Mts.], Laschkarak Tal [= Lashgarak valley], $1800 \mathrm{~m}$ a.s.1., vii.-X.1961, 1 \3 3 \% , J. Klapperich lgt., P. Kment det. (NMPC). ZanJan: 7 km E Sa'id Abad, $36^{\circ} 23.3^{\prime} \mathrm{N}$ $48^{\circ} 11.7^{\prime} \mathrm{E}, 1635 \mathrm{~m}$ a.s.1., 11.-12.v.2008, steppe stream, at light, 19 , J. Hájek \& P. Chvojka lgt., P. Kment det. (NMPC). OMAN: NE, 17 km W of Sur, 15.iii.2015, 1 \& M. Snížek lgt., P. Kment det. (ZJPC). TURKEY: AdIyaman: Burmapinar env., bank of river in canyon above Septimus Severus bridge, $37^{\circ} 55^{\prime} 57^{\prime \prime} 38^{\circ} 36^{\prime} 31^{\prime \prime}, 704 \mathrm{~m}$ a.s.1., 3.vii.2011, 2 of 1 L1 1 L2, P. Kment lgt. \& det. (NMPC). ANTALYa: Köprülü canyon, Beşkonak, 80 km NE of Antalya, 7.ix.1992, 3 त 1 L, Z. Jindra lgt., P. Kment det. (ZJPC). Tunceli: Ovacik env., bank of Munzur river downstreams the village before entering Munzur Vadisi Milli Parki, 4.vii.2011,

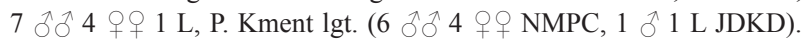
Afrotropical Region: ANGOLA: Angola (No. 10238.12), 1 ㅇ, P. Kment det. (NMPC). Lunda NorTe: Luachimo (Ang. No. 915.6), 11.vii.1948, 1 \& 1 , A. B. Machado lgt., P. Kment det. (NMPC); Luachimo (M) (Ang. No. 3967.2 ), 6.vi.1959, 1 ₹ 9 \% , Mus. do Dundo, P. Kment det (NMPC); Luachimo, Route Tourismo, M. H. B (Ang. No. 20175), 28.vi.1957, 1 9, Mus. do Dundo, P. Kment det. (NMPC). Lunda Sul: Alto Chicapa, Camutongola river, Luhembe (M) (Ang. No. 4174.7), 1 , 22.vi.1954, Mus. do Dundo, P. Kment det. (NMPC); Alto Chicapa, Camutongola river, Luhembe (M) (Ang. No. 4166.10, 4168.7, 4181), 11 J) 11 우, 24.vi.1954, Mus. do Dundo, P. Kment det. (NMPC); Alto Chicapa, Tehissango / Cuango Muquè (Ang. No. 4308.14), 1 , 16.vii.1954, Mus. do Dundo, P. Kment det. (NMPC); Alto Cúlo (M), Cavuemba (Ang. No. 4082.24), 31.v.1954, 3 q , Mus. do Dundo, P. Kment det. (NMPC). CENTRALAFRICAN REPUBLIC: $90 \mathrm{~km} \mathrm{NNE}$ Bangui, $5^{\circ} 03^{\prime} \mathrm{N} 18^{\circ} 47^{\prime} \mathrm{E}, 380 \mathrm{~m}$ a.s.1., 14.iii.2010, 1 §े, J. Halada lgt., P. Kment det. (MMBC). DEMOCRATIC REPUBLIC OF THE CONGO: Kasai OCcidental: Tshikapa (Ang. No. 880.4), vi.-vii.1948, $2 \lesssim 1 \%$, A. B. Machado lgt., P. Kment det. (NMPC). NIGERIA: TARABA: Gashaka Gumti NP, $30 \mathrm{~km}$ SE Serti env., $7^{\circ} 21^{\prime} \mathrm{N} 11^{\circ} 32^{\prime} \mathrm{E}$, ca. $450 \mathrm{~m}$ a.s.1., 24.iv. -8.v.2011, 1 \% 1 , J. Halada lgt., P. Kment det. (ZJPC). SUDAN: SEnNAR: Blue Nile prov., Dinder Game Reserve, Galegu, 4.iii.1967, 1 J 2 , 9 , P. Štys lgt. (686), P. Kment det. (MMBC). Oriental Region: INDIA: Maharashtra: $10 \mathrm{~km}$ E from Savantvadi, 22.v.2006, $2 \hat{\jmath}, \mathrm{Z}$. Kejval lgt., P. Kment det. (MMBC); $15 \mathrm{~km} \mathrm{E}$ of Savantvadi, $15^{\circ} 55^{\prime} \mathrm{N}$ $75^{\circ} 53^{\prime} \mathrm{E}, 40 \mathrm{~m}$ a.s.1., riverside, 22.v.2006, 1 §े, Z. Kejval lgt., P. Kment det. (MMBC); $40 \mathrm{~km} \mathrm{~W}$ of Pune, Mulshi env., 30.ix.-2.x.2005, 1 \&, J. Bezděk lgt., P. Kment det. (NMPC); Western Ghats Mts., Panchgani, Wai env., 3.-5.vi.2008, $2 \lesssim 1$ \&, Z. Kejval lgt., P. Kment det. (MMBC). Puducherry: Karaikal, Kurumbagaram, 23.iii.1947, 48 ते 25 우 Nathan lgt., L. Hoberlandt det., P. Kment revid. (43 24 5 jo 1 MMBC). Rajasthan: $20 \mathrm{~km} \mathrm{~N}$ of Dausa, Golakabas env. Sarsadevi, $27^{\circ} 05.46^{\prime \prime} \mathrm{N} 78^{\circ} 17.18^{\prime \prime} \mathrm{E}$ [sic!], $380 \mathrm{~m}$ a.s.1., 19.xi.2002, P. Šípek lgt., P. Kment det. (NMPC); $20 \mathrm{~km} \mathrm{~N}$ of Dausa, Naranimata env., $27^{\circ} 05.46^{\prime \prime} \mathrm{N} 78^{\circ} 17.18^{\prime \prime} \mathrm{E}$ [sic! ], $400 \mathrm{~m}$ a.s.1., light trap, 7.-8.xi.2002, 1 은, P. Šípek lgt., P. Kment det. (NMPC); $50 \mathrm{~km} \mathrm{~W}$ of Agra, Bharatpur env., $27^{\circ} 12.42^{\prime} \mathrm{N} 77^{\circ} 30.48^{\prime \prime} \mathrm{E}, 220 \mathrm{~m}$ a.s.1., 31.viii.-5.ix.2002, 1 1 L5, P. Šípek \& M. Fikáček lgt., P. Kment det. (NMPC). TAMIL NADu: Madras occ., Tiruchirappalli, 16.v.1970, 1 †, J. Hloušek lgt., P. Kment det. (MMBC). LAOS: Vientiane: Phou Khao Khouay NBCA, ca. $50 \mathrm{~km} \mathrm{~N}$ Vientiane (lake env.), $18^{\circ} 23.2^{\prime} \mathrm{N} 102^{\circ} 45.5^{\prime} \mathrm{E}, 745 \mathrm{~m}$ a.s.1., 18.v.2010, 1 o, J. Hájek lgt., P. Kment det. (NMPC); Vang-Veng, $18^{\circ} 55^{\prime} 23^{\prime \prime} \mathrm{N}$ $102^{\circ} 26^{\prime} 53^{\prime \prime}, 300$ m a.s.1., 10.-15.v. \& 1.-6.vii.2001, 1 đ̊, J. Kolibáč lgt., P. Kment det. (MMBC). MALAYSIA: SABAH: Mt. Kinabalu env., 28.v.1999, M. Snížek lgt., P. Kment det. (ZJPC); Sapulut env., 1.-6. vi.2001, 1 ๆ, J. Stolarczyk lgt., P. Kment det. (ZJPC); W, Crocker Range E, W of Apin Apin, v.1999, 1 Jे, M. Snížek lgt., P. Kment det. (ZJPC).
SARAWAK: Kapit distr., Rumah Ugap env., Sut river valley, 3.-9.iii.1994, 2 우, P. Bílek lgt., P. Kment det. (ZJPC); Kapit distr., Sebong, Baleh river, 6.-21.iii.1994, 2 ô, S. Bílý lgt., P. Kment det. (NMPC). PAKISTAN: PunJab: Rawalpindi env., Basal, Kalachitta Range, 4.-7.i.1956, 1 ${ }^{2}$, Chr. Lindemann lgt., L. Hoberlandt det., P. Kment revid. (NMPC). SRI LANKA: Central Province: Sigirya near Dambulla, Pidurangala

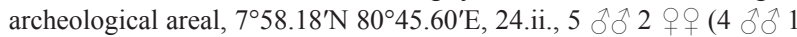
Z ZJPC, $1 \curvearrowright 1$ NMPC). RATNAPURA: Panamure env., 11.xii.1995, 1 †, S. Bečvář lgt., P. Kment det. (MMBC). Southern Province: Tissamaharama env., 90 km NE of Matara, 14.iii.1994, 1 ₹ 1 q, Z. Kejval lgt., P. Kment det. (ZJPC). THAILAND: ChIANG MaI: Doi Suthep Mt., 1.v.1996, 1 đ̃, S. Bílý lgt., P. Kment det. (NMPC). SAtun: Thale Ban, $6^{\circ} 45^{\prime} \mathrm{N} 100^{\circ} 09^{\prime} \mathrm{E}, 200 \mathrm{~m}$ a.s.1., 8.-13.iv.1997, 1 †, J. Kolibáč lgt., P. Kment det. (MMBC).

Distribution. Palaearctic Region: Europe: Bulgaria (JosIFOV 1954, 1960, 1963; GÖLLNER-SCHEIDING \& ARNOLD 1988; Stoianova \& Simov 2016); France (Latreille 1804, 1807; Dufour 1833; Burmeister 1835; Brullé 1836; АMyot \& Serville 1843; Puton 1880; Poisson 1925, 1957; de SeABra 1926, JACZEWSKi 1934; Rieger 1976, 1977; Elder 2017); Gibraltar (MAYr 1868, Hoffmann 1941); Greece (Josifov 1959, Zimmermann 1982, Polhemus \& Polhemus 2012); Italy, incl. Sicily (Costa 1862, Mancini 1952, SERvadei 1967, TAmanini 1981, Cianferoni \& Terzani 2013); North Macedonia (Protić 1998); Portugal (de SeAbra 1926, NieSer \& Montes 1984, Baena \& VÁzquez 1986, HebsgaArd et al. 2004); Russia (Daghestan) (Kanyukova 1997, 2006); Spain (Dufour 1833; Burmeister 1835, de Seabra 1926, Lindberg 1929, Baena Ruiz \& Ferreras Romero 1982, Nieser \& Montes 1984, Baena \& VÁzquez 1986, García Rojas et al. 1987, Nieser \& Chen 1992, Pagola-Carte \& Ribes 2007, GÜNTHER \& GÜNTHER 2019); Turkey: European Part (Fent et al. 2011, Fent \& Dursun 2018). North Africa: Algeria (Sienkiewicz 1964, Eckerlein \& Wagner 1965); Egypt (Priesner \& Alfieri 1953, LinNAVuori 1964); Morocco (LindBerg 1929, VidAl 1937, Aguesse et al. 1982, Gheit 1995, L'Mohdi et al. 2008); Tunisia (LindBerg 1922, Carapezza 1997, Slimani et al. 2015). Asia: Afghanistan (China \& Miller 1950); Armenia (Akramovskaya 1959, KANYUKOVA 2006); Azerbaijan, incl. Nakhichevan Republic (Kolenati 1857; HoRvÁth 1899; KiritshenKo 1918, 1938; Kanyukova 2006); China: Anhui (new record), Beijing (Nieser \& Chen 1992, Chen 1999, Xie \& Liu 2013), Fujian (Kormilev 1971, Nieser \& Chen 1992, Chen 1999, HuA 2000, XIE \& Liv 2013), Guangdong (HofFMANN 1933, 1941; Nieser \& Chen 1992; Chen 1999; Hua 2000; Chen et al. 2001; XIE \& LiU 2013), Guizhou (NIESER \& CHEN 1992, Chen 1999, Xie \& Liu 2013), Hainan (Hoffmann 1933, 1941; Nieser \& Chen 1992; Chen 1999; XIE \& Liu 2013), Hebei (Liu \& Bu 2009), Henan (SHEN et al. 2014, CAI et al. 2016), Hong Kong (Hoffmann 1941, Kormilev 1971), Hubei (Nieser \& Chen 1992, Chen 1999, XIE \& Liu 2013), Hunan (Nieser \& Chen 1992, Chen 1999, XIE \& Liu 2013), Inner Mongolia (NIESER \& Chen 1992, Chen 1999, XIE \& Liu 2013), Jiangsu (Nieser \& Chen 1992, Xie \& Liu 2013), Jiangxi (Zhang 1994, Hua 2000, Li et al. 2008), Shanxi (Mічамото 1960, as O. m. flavomarginatus), Shaanxi (new record), Sichuan (NiEser \& CHEN 1992, CHEN 1999, HuA 2000, XIE \& Liu 2013), Tianjin (NIESER \& CHEN 1992, CHEN 1999), Xizang (= Tibet) (HuA 2000), Yunnan (Cheng et al. 2006), Zhejiang (Kormilev 1971, Nieser \& Chen 1992 , 
Chen 1999, Xie \& Liu 2013); Cyprus (Lindberg 1948, Georghiou 1977, Polhemus \& Polhemus 2012); Georgia (KiRITSHENKo 1918, KanyuKova 2006); Iran (LinNAVUORI \& Hosseini 2000, Ghahari et al. 2013); Israel (Polhemus \& Polhemus 2012); Japan (ScotT 1874, as P. flavomarginatus): Hokkaido (HAYASHI 1995, as P. flavomarginatus; Hayashi et al. 2016; Hayashi \& MiYamoto 2018), Honshu (Matsumura 1905, 1915, as P. flavomarginatus; HiURA 1967, as O. m. flavomarginatus; Kormilev 1971; ToMoKUNI 1981, as O. m. flavomarginatus; HAYASHI et al. 2016; Hayashi \& Miуamoto 2018), Kyushu (Matsumura 1905, 1915, as P. flavomarginatus; JACZEWSKI 1934; KoRMILEV 1971; HiURA 1967, as O. m. flavomarginatus; TAKENO 1998; Hayashi et al. 2016; Наyashi \& MiYamoto 2018), Ryukyu Islands (МіYAмото 1964, HaYASHi et al. 2016; Hayashi \& Miуamoto 2018), Shikoku (Hivra 1967, as $O$. m. flavomarginatus; YANO et al. 2012; HAYASHI et al. 2016; HAYASHI \& MIYAMOTO 2018), Tanegashima Islands (HAYASHI \& Miуамото 2018); Jordan (КАтвен et al. 2000); Korea (Miyamoto \& Lee 1966, Josifov \& Kerzhner 1972, Lee \& Kwon 1991, Lee et al. 1993, LeE et al. 2013); Lebanon (LindBerg 1922); Oman (new record); Russia: Far East: Kunashir Island (Kerzhner 1978, Kerzhner \& MARUsiK 1997, KeRZHNER et al. 2004, KANYUKOva 2006, KANYUKOva \& Marusik 2006, Vinokurov et al. 2010); Saudi Arabia (Brown 1951, Linnavuori 1986); Syria (Fieber 1861, Poisson 1934, Nieser \& Moubayed 1985); Taiwan (MatSUMURa 1915, as P. formosanus; Bergroth 1914; EsaKI 1926; JACZewSKi 1934, KoRmilev 1971, XIE \& LiU 2013); Turkmenistan (KiRITSHENKo 1957, KanyukOVA 2006); Turkey (Asian part) (LindBerg 1922, HoberLandt 1952, FEnT et al. 2011, MAtocQ et al. 2014, ÖzGEN et al. 2017, Çerçi et al. 2018, Dursun \& Fent 2018, Fent \& Dursun 2018); United Arab Emirates (LinNAVUORI et al. 2011). Afrotropical Region: Angola (KORMILEV 1973); Botswana (KRÜGER \& DeCKerT 2016); Burkina Faso (LinNAVuori 1981); Cameroon (Kormilev 1973); Cape Verde Islands (LindBERG 1959); Central African Republic (new record), Democratic Republic of the Congo (Kormilev 1973); Ethiopia (JACZEWSKi 1934, Brown 1951); Gambia (NIESER \& Chen 1992); Guinea-Bissau (Mancini 1939a); Ivory Coast (Linnavuori 1981); ? Kenya (Montandon 1914); Mozambique (Poisson 1934); ? Namibia (Hesse 1925); Nigeria (Kormilev 1973, Linnavuori 1981); ? Senegal (SienKIEWICZ 1964); South Sudan (LinNAVuORI 1980); Sudan (new record); Tanzania (Nieser \& Chen 1992, PolHemus \& Polhemus 2012); ? Uganda (Montandon 1914); Yemen (BRown 1951, LinNAVUORI 1989). Oriental Region: India (Distant 1906): Assam (Thirumalai 2007, Polhemus \& Polhemus 2012), Bihar (Maxwell-Lefroy 1909, Distant 1910, Katiyar 1953, Thirumalai 2007), Kerala (Distant 1910, Thirumalai 2007), Madhya Pradesh (Thirumalai 2007, Thirumalai et al. 2007), Maharashtra (Paiva 1919b; Jaczewski 1934; Thirumalai \& Sharma 2002, 2012), Manipur (BAL \& Basu 2004, Thirumalai 2007), Meghalaya (Paiva 1919a, Bal \& Basu 1998, Thirumalai 2007), Mizoram (BAL \& BAsu 2007), Orissa (Distant 1910, Ghosh et al. 1989, Thirumalai 2007), Puducherry: Karaikal (KorMiLev 1971, Thirumalai 2007), Rajasthan (new record),
Tamil Nadu (Katiyar 1953; Sienkiewicz 1964; Thirumalai 1989, 1999, 2001, 2007; Polhemus \& Polhemus 2012), Tripura (BAl \& Basu 2000), Uttar Pradesh (Distant 1910, Thirumalai 2007), West Bengal (Distant 1910, Thirumalai 2007, Polhemus \& Polhemus 2012); Indonesia: Bali (Schouteden 1933, Polhemus \& Polhemus 2012), Java (JACZEWSki 1935, Kormilev 1971), Sumatra (JACZEWSKi 1934, Mancini 1939a); Laos (KoRmilev 1971); Malaysia: Peninsular part (Kormilev 1971, KovaC \& YANG 1989, Polhemus \& Polhemus 2012), Sabah (Kormilev 1971; Nieser \& Chen 1992, 1999), Sarawak (Kormilev 1971); Myanmar (Distant 1910, JACZEWSKI 1934); Nepal (DisTANT 1910); Pakistan (Hamid \& Hamid 1973); Singapore (Polhemus \& Polhemus 2012); Sri Lanka (Distant 1910, as $P$. marginatus, 1915, as $S$. rutherfordi; JACZEWSKI 1934; Polnemus 1979; Polnemus \& Polhemus 2012); Thailand (Kormilev 1971, Polhemus \& Polhemus 2012); Vietnam (Distant 1906, JACZEwSKi 1934, as Cochinchine; Kormilev 1971, Polhemus \& Polhemus 2012). Australasian Region: Indonesia: Sulawesi (NIESER \& CHEN 1999).

Notes. Polmemus (1995a) listed O. marginatus from Bosnia and Herzegovina and Croatia, however, there are no such records in the relevant literature (cf. Josifov 1986, PROTIĆ 1998, KMENT \& BERAN 2011) and taking into account its confinement to the southernmost regions of Balkan Peninsula (Josifov 1986, Stoianova \& Simov 2016) they are probably based on misinterpretation of records from former Yugoslavia. The records of $O$. marginatus from Australia (KIRKALdy 1908, based on a larva; Hale 1925), Tasmania, New Guinea and New Caledonia (MonTANDON 1897) belong to other species. The record from New Caledonia (Distant 1920 ) belong to O. australicus (JACZEWSKI 1934). The records of $O$. marginatus from Kenya, Uganda (MonTANDON 1914), Namibia (Hesse 1925) or Senegal (SienKIEwICZ 1964) requires confirmation. The records from Myanmar by Montandon (1897) and Distant (1906) refer to O. feae.

Polhemus \& Polhemus (2012) discussed variability of Oriental populations of $O$ marginatus, finding the male genitalia of specimens from Malaysia and Singapore structurally similar to those from India and the west Palaearctic Region. The populations from Bali are smaller and have also slightly different shapes of the superior appendage on the right paramere and the process of the male pygophore. They wrote that these more eastern populations may be assignable to the subspecies $O$. marginatus insularis from Philippines, but an analysis of further material from throughout the Malay Archipelago region will be necessary to properly determine the subspecific designations that should be applied to these insular forms (Polhemus \& Polhemus 2012). According to N. Nieser and P.-p. Chen, O. marginatus in its present sense represents rather a complex of several species (CHEN et al. 2005). In this paper we identify $O . m$. marginatus as a polymorphic species in the sense of JACZEWSKI (1934), KoRmilev (1973) and Polhemus \& Polhemus (2012). However, we observed considerable differences in the shape of the parameral cap and the parameral appendages among the populations, e.g when comparing specimens from tropical Africa, Mediterranean and south India, and agree with the above-mentioned opinion of CHEN et al. (2005). 


\section{Ochterus marginatus insularis Rieger, 1977}

Ochterus marginatus insularis Rieger, 1977: 213-214 (description, differential diagnosis, illustrations). HolotyPE: $\hat{\delta}$, Philippines: Mindanao: 'Süd [= South] Mindanao, Port Banga' (ZSMC).

Ochterus marginatus (partim): KoRMILEV (1971): 435-436 (partim, distribution); GAPUD \& SAN VALENTIN (1977): 271, 273-276, 281, 285-286, 288-289, 294, 296-298 (key to species, redescription, illustrations, variation); GAPUD (1981): 301-302 (taxonomy); GAPUD (1986): 32-34 (key to species, redescription, illustration); NIESER \& CHEN (1999): 91 (partim, distribution); CHEN et al. (2005): 413 (checklist); PolHEMus \& Polhemus (2012): 345 (partim, distribution).

Ochterus marginatus insularis: GAPUD (2003): 107 (checklist); PolHEMUS \& Polhemus (2012): 345, 347 (variability).

Distribution. Oriental Region: Philippines: Cebu (GAPud 2003), Leyte (GAPUd 1981), Luzon (Kormilev 1971, Rieger 1977, Gapud \& SAN Valentin 1977, Yano et al. 1981, Nieser \& Chen 1999, Polhemus \& Polhemus 2012), Mindanao (Rieger 1977, Gapud 1981, Nieser \& Chen 1999, Polhemus \& Polhemus 2012), Mindoro (Kormilev 1971, Gapud \& San Valentin 1977), Palawan (Kormilev 1971, GAPUD 2003), Romblon (GAPUD 1981).

Note. According to GAPUD (1981), out of the twelve individuals he examined, all except one agreed with the diagnosis of O. marginatus insularis by RIEGER (1977). He considered difficult to assess whether these characters are sufficient for a subspecies delimitation. According to Polnemus \& Polmemus (2012), the population of $O$. marginatus from Bali may also belong to this subspecies (see Notes under O. marginatus marginatus).

\section{Ochterus mexicanus}

\section{A. Polhemus \& J. T. Polhemus, 2016}

Ochterus mexicanus D. A. Polhemus \& J. T. Polhemus, 2016: 17, 44-47 (key to species, description, illustrations). Holotype: $\hat{\sigma}$, Mexico: 'Sinaloa, Rio Quelite at El Quelite [2332'11"N, 106²8‘46"W, elev. $25 \mathrm{~m}]$ ' (JTPC $\rightarrow$ USNM).

Distribution. Neotropical Region: Central America: Mexico: Colima (Polnemus \& Polnemus 2016), Guerrero (Polnemus \& Polhemus 2016), Jalisco (Polnemus \& Polhemus 2016), Morelos (Polhemus \& Polhemus 2016), Oaxaca (Polhemus \& Polhemus 2016), Puebla (Polhemus \& Polhemus 2016), Sinaloa (Polhemus \& Polhemus 2016).

\section{Ochterus minor Kormilev, 1973}

Ochterus minor Kormilev, 1973: 2-4-5, 8 (description, differential diagnosis, illustrations). Hоцотур: $\partial$, Democratic Republic of the Congo: 'CONGO, Matadi' (CASC).

Material examined. ANGOLA: Bı́́: Catabola, 17.-27.xi.2012, FIT [= flight interception trap], $1 \hat{\jmath}$, T. Lackner lgt. MMBC). CunENE: Chuttes de Ruacana [= Ruacana Falls], (Ang. No. 1903.3), 6.x.[19]49, 1 d, A. B. Machado lgt. (NMPC). All. P. Kment det.

Distribution. Afrotropical Region: Angola (KORMILEV 1973); Burkina Faso (LINNAVUORI 1981); Benin (LINNAVUORI 1981); Chad (Linnavuori 1981); Democratic Republic of the Congo (Kormilev 1973); Ivory Coast (Linnavuori 1981); Nigeria (Kormilev 1973, LinNAVuori 1981).

\section{Ochterus monteithorum Baehr, 1990}

Ochterus monteithorum Baehr, 1990b: 451, 453, 454, 456-459, 461, 468 (key to species, description, illustration). Holotype: $\hat{\jmath}$, Australia: Queensland: 'Leo creek Road, McIllwraith Range, $30 \mathrm{~km} \mathrm{NE.} \mathrm{of}$ Coen, 500 m' (QMBA).

Ochterus monteithorum: CASSIS \& Gross (1995): 260 (catalogue); ANDERSEN \& WeIR (2004): 267-268, 270, 335 (key to species, illustrations, checklist).

Distribution. Australian Region: Australia: Queensland (BAEHr 1990b, ANDERSEN \& WeIr 2004).

\section{Ochterus nicobarensis Chandra \& Jehamalar, 2012}

Ochterus nicobarensis Chandra \& Jehamalar, 2012: 24-26 (description, illustrations). Ноцотүе: $\precsim$, India: Nicobar Islands: 'Nicobar District, Great Nicobar Biosphere Reserve, Campbell Bay, Govind Nagar, Ring Road, attracted to light near a pond $0.5 \mathrm{~km}$ away from sea, $40 \mathrm{ft}$. [12 $\mathrm{m}$ a.s.1.], $7^{\circ} 00.075^{\prime} \mathrm{N} 93^{\circ} 54.594^{\prime} \mathrm{E}^{\prime}$ (NZSI).

Material examined. MYANMAR: Burma, Rangoon Distr., Hlegu Goygon, v.1997, 1 § 1 , M. Klícha lgt., P. Kment det. (ZJPC).

Distribution. Oriental Region: India: Nicobar Islands (Chandra \& Jehamalar 2012); Myanmar (new record).

\section{Ochterus nigrinus Baehr, 1990}

Ochterus nigrinus Baehr, 1990b: 451, 453-454, 456-459, 461, 464-465 (key to species, description, illustrations). Holotype: 2 , Solomon Islands: 'Guadalcanal, Monitor Creek, Umasami R.' (BMNH). Ochterus nigrinus: CHEN et al. (2005): 413 (checklist).

Distribution. Australian Region: Solomon Islands: Guadalcanal (BAEHR 1990b)

\section{Ochterus noualhieri Baehr, 1990}

Ochterus noualhieri Baehr, 1990c: 91-93 (key to species, description, illustrations). Holotype: đે, Indonesia: Java: 'Java Occ., Volc. Géde' (MNHN).

Ochterus noualhieri: NIESER \& CHEN (1999): 90-91 (key, illustrations); Chen et al. (2005): 413 (checklist); Polhemus \& Polhemus (2012): $345,352,354-356$ (key to species, diagnosis, illustration); PolHEMUs \& Polhemus (2015): 208-209, 212 (key to species, illustrations).

Distribution. Oriental Region: Indonesia: ? Bali (NIESER \& Chen 1999, female only), Java (BAeHr 1990c). Australian Region: Indonesia: Sulawesi (NIESER \& CHEN 1999, Polhemus \& Polhemus 2012).

\section{Ochterus obscurus}

\section{A. Polhemus \& J. T. Polhemus, 2016}

Ochterus obscurus D. A. Polhemus \& J. T. Polhemus, 2016: 15-16, 48-50 (key to species, description, illustrations). Ноцотүре: $\hat{\jmath}$, Panama: 'Chiriqui, $5.6 \mathrm{~km} \mathrm{~N}$ of Boquete, La Culebra Trail, 1800 m’ (USNM).

Distribution. Neotropical Region: Central America: Costa Rica (Polhemus \& Polhemus 2016); Panama (Polhemus \& Polhemus 2016).

\section{Ochterus occidentalis Baehr, 1990}

Ochterus occidentalis Baehr, 1990b: 452-454, 456-460-462 (key to species, description, illustrations). Hоцотуре: $\partial$, Australia: Western Australia: 'Margaret River' (BMNH).

Ochterus occidentalis: CASSIS \& GROSS (1995): 261 (catalogue); ANDERSEN \& WeIR (2004): 268, 270, 335-336 (key to species, illustrations, checklist). 
Distribution. Australian Region: Australia: Western Australia (BAeHr 1990b, ANDERsen \& WeIr 2004).

\section{Ochterus ovatus}

\section{A. Polhemus \& J. T. Polhemus, 2016}

Ochterus ovatus D. A. Polhemus \& J. T. Polhemus, 2016: 17, 50-53 (key to species, description, illustrations). Ноцотуре: ${ }^{2}$, Mexico: 'Chiapas, Soyalo, Rio Blanco, on road to Villahermosa 14 mi. N of Hwy. 190, small swift mountain river with backwater pools [vic. $16^{\circ} 53^{\prime} 27^{\prime} \mathrm{N}$, 9255'30“W, elev. $\sim 1200 \mathrm{~m}$ ]' (JTPC $\rightarrow$ USNM).

Distribution. Neotropical Region: Central America: Mexico: Chiapas (Polhemus \& Polhemus 2016).

\section{Ochterus panamensis}

\section{A. Polhemus \& J. T. Polhemus, 2016}

Ochterus panamensis D. A. Polhemus \& J. T. Polhemus, 2016: 13, 18, 53-55 (key to species, description, illustrations). HoLотуPE: $\precsim$, Panama: 'Panama, small stream in limestone bed at Serrania de Majé, $3 \mathrm{~km}$ S of Ipete, $100 \mathrm{~m}$ [vic. $\left.8^{\circ} 57^{`} 37^{\prime \prime} \mathrm{N}, 78^{\circ} 31^{\prime} 17^{\prime \prime} \mathrm{W}\right]$ ' (JTPC $\rightarrow$ USNM).

Distribution. Neotropical Region: Central America: Panama (Polhemus \& Polhemus 2016).

\section{Ochterus papaceki Kment \& Carapezza sp. nov.}

Ochterus papaceki Kment \& Carapezza, sp. nov. (original description, illustrations). Ноцотуре: $\hat{\text { d }}$ Yemen: Socotra Island: 'Noged plain, lower part of Wadi Matyaf, $12^{\circ} 27^{\prime} 13.50^{\prime \prime} \mathrm{N} 54^{\circ} 18^{\prime} 13.95^{\prime \prime} \mathrm{E}^{\prime}$ (NMPC).

Distribution. Afrotropical Region: Tanzania; Yemen: Socotra (this paper).

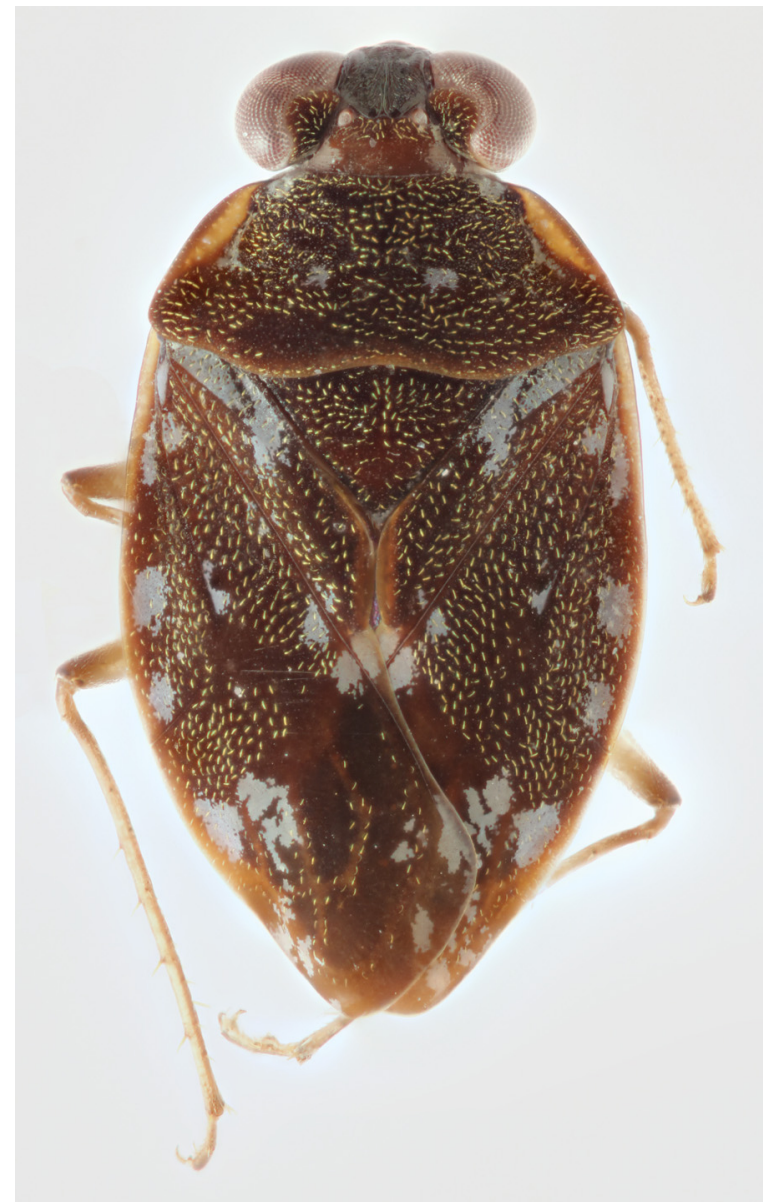

Fig. 24. Ochterus parvus Schell, 1943, female, Ecuador: Napo: San Pablo de Ushpayacu env., $3.73 \mathrm{~mm}$.

\section{Ochterus papuasicus Kormilev, 1972}

Ochterus papuasicus Kormilev, 1972: 585-586 (description, differential diagnosis, illustrations). HоLотYPE: 1 , Papua New Guinea: 'SE (PAPUA): Rouna, 300-500 m' (BPBM).

Ochterus papuasicus: Chen et al. (2005): 413 (checklist).

Distribution. Australian Region: Papua New Guinea (KoRMiLEv 1972).

\section{Ochterus pardalos Nieser \& Chen, 1999}

Ochterus pardalos Nieser \& Chen, 1999: 90, 93-94, 123 (description, differential diagnosis, illustrations). HoLOTYPE: $\partial$, Philippines: Mindanao: 'Lake Sebu area, trickle of water fed by seepage from 'Cold River" (NCTN).

Ochterus pardalos: GAPUD (2003): 107 (checklist); CHEN et al. (2005): 413 (checklist).

Distribution. Oriental Region: Philippines: Mindanao (Nieser \& Chen 1999, GAPud 2003).

\section{Ochterus parvus Schell, 1943}

(Fig. 24)

Ochterus parvus Schell, 1943a: 32, 35 (key to species, illustration). LeCtotype (designated by Schell 1943b: 40, as holotype): §ै, Ecuador: 'Mera' (SEMC).

Ochterus parvus: SCHELL (1943b): 39-40 (original description, differential diagnosis); DraKe (1952): 74 (checklist); Froeschner (1981): 61 (catalogue); HeCKMAN (2011): 460 (key to species, illustration); Cianferoni (2012): 67 (key to species).

Material examined. ECUADOR: NAPO: $7.5 \mathrm{~km}$ SE of Archidona, San Pablo de Ushpayacu env., $0^{\circ} 56^{\prime} 52^{\prime \prime} \mathrm{S} 77^{\circ} 45^{\prime} 01^{\prime \prime} \mathrm{W}, 540 \mathrm{~m}$ a.s.1., 23.xi.2006, 1 + (Fig. 24), M. Fikáček lgt., P. Kment det. (NMPC).

Distribution. Neotropical Region: South America: Ecuador (Schell 1943b, Drake 1952, Cianferoni 2012).

Note. The revision of American Ochterus including the description of $O$. parvus was published divided in two parts, the first published in January 1943 (Schell 1943a) and the second in April 1943 (Schell 1943b). The species was first introduced in the key, which is sufficient to make it available (ICZN 1999: Art. 13.1.1), without any mention of type specimens (ScHeLl 1943a: 32), while the original description including the holotype designation appeared in the second part (SCHELl 1943b: 39). As a holotype is defined as 'the single specimen upon which a new nominal species-group taxon is based in the original publication' (ICZN 1999: Art. 73.1), in this case, the subsequently designated 'holotype' must be considered the lectotype according to ICZN (1999: Art. 74.5).

\section{Ochterus paucistriatus Baehr, 1990}

Ochterus paucistriata Baehr, 1990b: 450, 453, 454, 456-459, 461, 468-469 (key to species, description, illustrations). HоLотуре: $\hat{\jmath}$, Indonesia: Papua: 'Dutch New Guinea: Cyclops Mts., Mt. Lina, 3500 ft. [= $1067 \mathrm{~m}$ a.s.1.]' (BMNH).

Ochterus paucistriata: CHEN et al. (2005): 413 (checklist).

Distribution. Australian Region: Indonesia: Papua (BAEHR 1990b, CHEN et al. 2005)

Note. Baehr (1990b) established the species name as $O$. paucistriata, citing it 11 times throughout the paper, but without providing any explanation of its etymology. CHEN et al. (2005) also accepted $O$. paucistriata as the correct original spelling. However, the species name is a compo- 
sed Latin adjective paucistriatus $(-a,-u m)$ and the generic name Ochterus is masculine. Therefore, we correct here the gender agreement of the name to O. paucistriatus (see ICZN 1999: Art. 31.2, 34.2).

\section{Ochterus perbosci (Guérin-Méneville, 1843)}

Pelogonus Perboscii Guérin-Méneville, 1843: 113-114 (original description, differential diagnosis). LECTOTYPE (designated by CHAMPION 1901: 345, as type): A, Mexico: Campeche: 'dans la baie Campèche' [= in the Campeche bay] (coll. Sallé $\rightarrow$ BMNH).

Pelogonus Perboscii: FieBer (1851): 15 (checklist); Dohrn (1859): 52 (list); STÅL (1876): 137 (catalogue).

Pelogonus perbosci: Champion (1901): 344-345, Pl. XX: fig. 11 (key, diagnosis, illustration).

Ochterus perbosci(i): TORRe Bueno (1906): 50 (list).

Ochterus perboscii: KirKaldy \& TORRE Bueno (1909): 179 (catalogue). Pelogonus Perbosci: Montandon (1910): 1 (note).

Ochterus perbosci: BARBER (1913): 213 (key to species); VAN DuzeE (1923): 167 (distribution); Schell (1943a): 32-33, 35 (key to species, original description repeated, differential diagnosis, illustration); DraKe (1952): 75 (checklist); COBBEN (1960): 54 (distribution); NiESER (1975): 26, 28-29, 31, Pl. 2a (key to species, diagnosis, illustrations); Polhemus (1976): 226, 242 (listed); Froeschner (1981): 61 (catalogue); Polhemus \& Polhemus (1988a): 543 (catalogue); Nieser \& AlKINS KoO (1991): 13-14 (key); NIESER \& CHEN (1992): 8 (distribution); Nieser \& MELo (1997): 12, 50 (distribution); FroESCHNER (1999): 148 (catalogue); Bass (2003): 66 (distribution); Vianna \& Melo (2003): 126-127 (distribution); Melo \& Nieser (2004): 44 (distribution); Souza et al. (2006): 808 (distribution); Pereira \& Melo (2007): 645 (distribution); HeCKMAN (2011): 458 (key to species, illustration); Moreira et al. (2011): 58 (checklist, Brazil); CiAnFeroni (2012): 67 (key to species); BrożeK (2013): 342, 343, 345, 372 (morphology); Cordeiro et al. (2014): 496 (key to species, illustrations); BARBosa \& Rodrigues (2015): 163 (listed); BRożeK (2015): 6, 14, 21-24 (morphology); Llano \& Gutiérrez (2015): 377 (list); Polhemus \& Polhemus (2016): 13, 15-16, 55-58 (key to species, redescription, illustrations).

Pelogonus marginatus (misidentification, partim): UHLER (1893): 706 (list, 'two forms'); UhLER (1894): 222 (variability).

Material examined. MEXICO: BaJa California Sur: [El] Triunfo, 8.vii.1914, 1 , G. H. Harris lgt., C. J. Drake det. (NMPC). San Luis Potosí: Valles, 17.vii.1950, $2 \lambda 1$, Drake \& Hottes lgt., C. J. Drake det. (NMPC). Tamaulipas: Tampico, 16.vii.1950, 1 ô, Drake \& Hottes lgt., C. J. Drake det. (NMPC)

Distribution. Nearctic Region: North America: USA: Arizona (Drake 1952, Polhemus \& Polhemus 1988a), Texas (Schell 1943a; Drake 1952; Polhemus \& Polhemus 1988a, 2016), Utah (Polhemus \& Polnemus 2016). Neotropical Region: Central America: Belize (Polnemus \& Polhemus 2016); Costa Rica (Schell 1943a); Guatemala (Schell 1943a, Polhemus \& Polhemus 2016); Honduras (Polhemus \& Polhemus 2016); Mexico (Kirkaldy \& Torre Bueno 1909, Schell 1943a, Drake 1952, Polhemus \& Polnemus 1988a): Baja California Sur (Polnemus \& Polhemus 2016), Campeche (ChAmpion 1901, Montandon 1910, Polhemus \& Polhemus 2016), Chiapas (Polhemus \& Polhemus 2016), Guanajuato (Polhemus \& Polhemus 2016), Guerrero (Polhemus \& Polhemus 2016), Jalisco (Polhemus \& Polhemus 2016), Mexico (Polnemus \& Polhemus 2016), Morelos (Polnemus \& Polhemus 2016), Nayarit (Polhemus \& Polhemus 2016), Oaxaca (Polhemus \& Polhemus 2016), Pueblo (Polhemus \& Polhemus 2016), San Luis Potosí (new record), Sinaloa (Champion 1901, Polhemus \& Polhemus 2016), Tamaulipas (Polhemus \& Polhemus 2016), Veracruz (Champion 1901, Polhemus \& Polhemus 2016); Nicaragua
(Polhemus \& Polhemus 2016); Panama (Polhemus \& Polhemus 2016). Caribbean Islands: Curaçao (CoBben 1960); Grenada (Uhler 1894, as P. marginatus, partim; Champion 1901, Kirkaldy \& Torre Bueno 1909, Schell 1943a, Polhemus \& Polhemus 2016); St. Vincent (Uhler 1893, as P. marginatus, partim; CHAMPION 1901, KIRKALDY \& Torre Bueno 1909, Schell 1943a, Polhemus \& Polhemus 2016); Trinidad and Tobago (NIESER \& Alkins Koo 1991, Nieser \& Chen 1992, BAss 2003). Souh America: Brazil: Amazonas (Pereira \& Melo 2007, Moreira et al. 2011, Cordeiro et al. 2014), Minas Gerais (Nieser \& Melo 1997, Vianna \& Melo 2003, Melo \& Nieser 2004, Souza et al. 2006, Moreira et al. 2011, CordeIro et al. 2014); Colombia (Drake 1952); Ecuador (Schell 1943a, Nieser \& Chen 1992, Cianferoni 2012); Paraguay (Schell 1943a); Peru (Schell 1943a, Drake 1952); Suriname (NIESER 1975); Venezuela (Polhemus \& Polhemus 2016).

Notes. GuÉRIN-MÉnEVILLE (1843) dedicated the species to its collector, M. Perbosc, using the latinized form of his name (Perboscius, stem perbosci-) to form the name as $O$. perboscii, which is the correct original spelling of the name. The name was cited as $O$. perboscii at least five times (for references see above) until KIRKALDY \& TORRE BuENO (1909), with a single exception of CHAMPION (1901). ChAmpion (1901), Montandon (1910), and all subsequent authors (for 27 additional references see above) used the incorrect subsequent spelling of the name, O. perbosci (see ICZN 1999: Art. 32.1-3, 33.3, 33.4). In this case the Article 33.3.1 (ICZN 1999) applies in favour of O. perbosci: 'when an incorrect subsequent spelling is in prevailing usage and is attributed to the publication of the original spelling, the subsequent spelling and attribution are to be preserved and the spelling is deemed to be a correct original spelling.' We recognize $O$. perbosci as being in prevailing usage and therefore the correct original spelling in the sense of ICZN (1999).

GuÉRIN-MÉNEVILLE (1843) described the species based on unknown number of specimens and without information on their depository. CHAMPION (1901) wrote that: 'The type of this insect was obtained by us from the Sallé collection'. CiANFERONi (2012) specified the sex of the specimen, incorrectly labelled as holotype, as male. Using the term 'the type' for the syntype he used for definition of the taxon, CHAMPION (1901) fulfilled the requirements of the Article 74.5 (ICZN 1999) and his action constitutes a valid lectotype designation.

It was also recorded from Cuba by CHAMPION (1901) and Kirkaldy \& Torre Bueno (1909) by error (see Naranjo et al. 2010, Polnemus \& Polmemus 2016).

\section{Ochterus philippinensis Kormilev, 1971}

Ochterus philippinensis Kormilev, 1971: 434, 436-437 (description, differential diagnosis, illustrations). НоцотүРE: $\mathcal{\partial}$, Philippines: Mindoro: 'San Jose' (CASC).

Ochterus philippinensis: GAPUD \& SAN VALENTIN (1977): 272, 281, 291-292 (key to species, redescription, illustration); GAPUD (1981): 303-304, 307 (diagnosis, illustrations); GAPUD (2003): 107 (checklist); CHEN et al. (2005): 413 (checklist).

Distribution. Oriental Region: Philippines: Mindoro (KoRMiLEV 1971, GAPUd \& SAN VALENTIN 1977, GAPUd 1981). 


\section{Ochterus piliferus Kormilev, 1973}

Ochterus piliferus Kormilev, 1973: 2, 6-8 (description, differential diagnosis, illustrations). Ноцотүре: $\hat{\jmath}$, Angola: 'Vila Luso, Moxico' (CASC).

Distribution. Afrotropical Region: Angola (KORMILEV 1973).

\section{Ochterus polhemusi Gapud, 1981}

Ochterus brunneus Gapud \& San Valentin, 1977: 272, 276-278, 281, 285-286, 288, 296-298 (key to species, description, illustrations). Holotype: 0 , Philippines: Luzon: 'Molawin Creek, Mt. Makiling, Laguna' (UPLB). Junior homonym of Ochterus brunneus Hungerford, 1927.

Ochterus brunneus: GAPUD (1986): 32, 34-36 (key to species, redescription, illustration).

Ochterus polhemusi Gapud, 1981: 300-301 (nomenclature). New substitute name for Ochterus brunneus Gapud \& San Valentin, 1977.

Ochterus polhemusi: GAPUD (2003): 107 (checklist); CHEN et al. (2005): 413 (checklist).

Distribution. Oriental Region: Philippines: Cebu (GAPUd 2003), Laguna (GAPud \& SAN VAlEntin 1977, GAPud 1981), Leyte (GAPud 1981), Mindanao (GAPud 1981), Mindoro (Gapud \& San Valentin 1977), Negros (GAPUD 2003).

\section{Ochterus pseudomarginatus}

\section{A. Polhemus \& J. T. Polhemus, 2012}

Ochterus pseudomarginatus D. A. Polhemus \& J. T. Polhemus, 2012 345-346, 349-351, 355 (key to species, description, illustrations) Holotyp: $\widehat{\partial}$, Malaysia: Johor: 'Endau Rompin, Sungai Pantai Burung, off Sungai Endau' (BPBM).

Ochterus pseudomarginatus: Polnemus \& Polnemus (2015): 208-209, 211 (key to species, illustrations).

Distribution. Oriental Region: Indonesia: Sumatra (Polhemus \& Polnemus 2012); Malaysia: Peninsular part (Polhemus \& Polhemus 2012); Singapore (Polhemus \& Polhemus 2012).

\section{Ochterus pseudorotundus}

\section{A. Polhemus \& J. T. Polhemus, 2016}

Ochterus pseudorotundus D. A. Polhemus \& J. T. Polhemus, 2016: 13, 18, 58-61 (key to species, description, illustrations). НоцотүРе: Mexico: 'Tamaulipas, Bocatoma, $7 \mathrm{~km}$ SSE of Gomez Farias [vic. $23^{\circ} 01^{\prime} 04^{\prime \prime} \mathrm{N}, 99^{\circ} 07^{\prime} 28^{\circ} \mathrm{W}$, elev. $135 \mathrm{~m}$ ]' (JTPC $\rightarrow$ USNM).

Distribution. Neotropical Region: Central America: Mexico: Nuevo León (Polhemus \& Polhemus 2016), San Luis Potosí (Polhemus \& Polhemus 2016), Tamaulipas (Polhemus \& Polhemus 2016), Veracruz (Polhemus \& Polhemus 2016).

\section{Ochterus rotundus}

\section{J. T. Polhemus \& M. S. Polhemus, 1976}

Ochterus viridifrons (misidentification): ScHELL (1943b): 38 (specimens from Grand Canyon); Drake (1952): 75 (record from Arizona) (see Polhemus \& Polhemus 1976: 223).

Ochterus rotundus J. T. Polhemus \& M. S. Polhemus, 1976: 223-225 (original description, illustrations). Holotype: $\hat{\sigma}$, Mexico: Durango: 'W Los Bancos, km 175, on top of divide' (JTPC $\rightarrow$ USNM).

Ochterus rotundus: Polhemus \& Polhemus (1988a): 542 (catalogue); Polmemus \& Polhemus (2016): 13, 18, 61-64 (key to species, redescription, illustrations, type depository).
Distribution. Nearctic Region: USA: Arizona (SCHELL 1943, as O. viridifrons; Polnemus \& Polnemus 1976, 1988a, 2016; Stevens \& Polhemus 2008); Mexico: Durango (Polhemus \& Polhemus 1976, 2016), Jalisco (Polhemus \& Polhemus 1976, 2016), Michoacan (Polhemus \& Polnemus 1976, 2016), Nayarit (Polhemus \& Polhemus 1976, 2016), Sinaloa (Polnemus \& Polhemus 1976, 2016), Sonora (Polnemus \& Polnemus 1976, 2016).

Note. Two series of female specimens from Jalisco (Mismaloya) and Nayarit (Compostela), identified tentatively as $O$. rotundus by Polnemus \& Polnemus (1976) probably belong to $O$. schellae (see Polhemus \& Polnemus 2016).

\section{Ochterus santosi Cordeiro \& Moreira, 2014}

Ochterus santosi Cordeiro \& Moreira, 2014 in CoRDEIRo et al. (2014): 494-496 (key to species, description, illustrations). HoLotyPE: §̊, Brazil: Piauí: 'Piauí-Piracuruca, Parque Nacional de Sete Cidades, Olho d'água dos milagres, $4^{\circ} 05^{\prime} 32^{\prime \prime} \mathrm{S} 41^{\circ} 40^{\prime} 48^{\prime \prime} \mathrm{W}, 180 \mathrm{~m}$ a.s.1.' (CZMA).

Distribution. Neotropical Region: South America: Brazil: Piauí (Cordeiro et al. 2014).

\section{Ochterus schellae Drake, 1952}

Ochterus acutangulus (misidentification): ScHELl (1943a): 32, 35 (key to species, illustration); Schell (1943b): 38-39 (differential diagnosis) (see Drake 1952, Polhemus \& Polhemus 2016).

Ochterus schellae Drake, 1952: 73, 75 (description). НоLотуре: Mexico: Mexico: Tejupilco, alt. 1340 m (coll. Drake $\rightarrow$ USNM).

Ochterus schellae: Drake (1952): 75 (checklist); Polhemus \& Polhemus (2016): 13, 18, 64-66 (key to species, redescription, illustrations).

Ochterus rotundus (misidentification): Polhemus \& Polhemus (1976): 223 (two series of female specimens from Jalisco (Mismaloya) and Nayarit (Compostela), tentatively identified as $O$. schellae by POLHEmus \& Polhemus 2016).

Distribution. Neotropical Region: Central America: Guatemala (Polhemus \& Polhemus 2016); El Salvador (Polnemus \& Polnemus 2016, females only); Mexico (Drake 1952): ? Jalisco (Polhemus \& Polhemus 2016, females only), Mexico (Drake 1952, Polhemus \& Polhemus 2016), ? Nayarit (Polnemus \& Polhemus 2016, females only), Sinaloa (Polnemus \& Polnemus 2016, females only).

\section{Ochterus secundus secundus Kormilev, 1971}

Ochterus secundus Kormilev, 1971: 434, 441-442 (description, differential diagnosis, illustrations). HoLoTYPE: $\hat{\alpha}$, Australia: New South Wales: 'N. S. W., N. Cronully [= Cronulla]' (AMSA).

Ochterus secundus: BAEHR (1989): 113, 116, 118, 123-124 (key to species, redescription, illustrations).

Ochterus secundus secundus: BAEHR (1990b): 450, 461, 475 (key to species, diagnosis, illustration, new status); CAssis \& GRoss (1995): 262 (catalogue); ANDERSEN \& WeIR (2004): 266-267, 270, 336 (key to species, illustrations, checklist).

Distribution. Australian Region: Australia: Australian Capital Territory (BAEHr 1990b, ANDERSEN \& WeIr 2004), New South Wales (Kormilev 1971, BaeHr 1989, Andersen \& WeIr 2004), Queensland (Kormilev 1971, BAeHr 1989, 1990b; ANDERSEN \& Weir 2004), South Australia (BAEHR 1989, ANDERSEN \& WeIr 2004), Victoria (Kormilev 1971; BAEHR 1989, 1990b; ANDERSEN \& Weir 2004), Western Australia (BAEHr 1989, ANDERSEN \& WeIr 2004). 
Ochterus secundus pseudosecundus Baehr, 1989

Ochterus pseudosecundus Baehr, 1989: 113, 114, 116, 118, 124-125 (key to species, description, illustrations). Ноцотуре: $\lambda$, Australia: Western Australia: 'Gascoyne River, $15 \mathrm{~km} \mathrm{~N}$. of Carnarvon, W. A.' (QMBA).

Ochterus secundus pseudosecundus: BAEHR (1990b): 450, 453, 455, 60, 461, 476 (key to species, diagnosis, illustration, new status); CAssis \& Gross (1995): 260 (catalogue); ANDERSEN \& WeIr (2004): 266-267, 270, 336 (key to species, illustrations, checklist).

Distribution. Australian Region: Australia: Western Australia (BAEHR 1989, 1990b; ANDERSEN \& WeIr 2004).

\section{Ochterus seychellensis D. A. Polhemus, 1992}

Ochterus seychellensis D. A. Polhemus, 1992a: 418-422 (description, illustrations). HoLотуре: $\hat{\jmath}$, Seychelles: Mahé: 'Mahe, seeping rock faces along Foret Noire road near Congo Rouge trailhead, 390 m' (USNM).

Ochterus seychellensis: Polnemus \& Polnemus (2008): 284, 286 (illustration, key to species); Polhemus \& Polhemus (2013): 94-95 (diagnosis, illustrations).

Distribution. Afrotropical Region: Seychelles: Mahé (Polhemus 1992a, Polhemus \& Polhemus 2013).

\section{Ochterus shepardi}

D. A. Polhemus \& J. T. Polhemus, 2016

Ochterus shepardi D. A. Polhemus \& J. T. Polhemus, 2016: 17, 66-69 (key to species, description, illustrations). HоLотуре: $\curvearrowright$, Costa Rica: ‘Alajuela, La Fortuna, Querado Burio’ (JTPC $\rightarrow$ USNM).

Distribution. Neotropical Region: Central America: Costa Rica (Polhemus \& Polhemus 2016).

\section{Ochterus signatus}

\section{A. Polhemus \& J. T. Polhemus, 2012}

Ochterus signatus D. A. Polhemus \& J. T. Polhemus, 2012: 345-347-349, 355 (key to species for Singapore, Malay Peninsula, Sumatra and Java; description, illustrations). HoLotype: of, Vietnam: Lam Dong Province: 'tributary stream to main river at Suoi Vang hydro, 1430

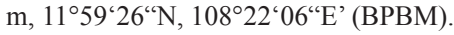

Ochterus signatus: Polhemus \& Polhemus (2015): 208-209, 211 (key to species, illustrations).

Distribution. Oriental Region: Malaysia: Peninsular part (Polhemus \& Polhemus 2012), Vietnam (Polhemus $\&$ Polhemus 2012).

\section{Ochterus singaporensis}

\section{A. Polhemus \& J. T. Polhemus, 2012}

Ochterus singaporensis D. A. Polhemus \& J. T. Polhemus, 2012: 345-346, 351-353, 355 (key to species, description, illustrations). HоLотуре: $\precsim$, Singapore: 'MacRitchie Catchment Reservoir, NS 123B \#G (stream towards reservoir)' (ZRCS).

Ochterus singaporensis: Polhemus \& Polhemus (2015): 207, 209-210 (key to species, illustrations).

Distribution. Oriental Region: Singapore (PolHemus \& Polhemus 2012).

\section{Ochterus smaragdinus}

D. A. Polhemus \& J. T. Polhemus, 2016

Ochterus smaragdinus D. A. Polhemus \& J. T. Polhemus, 2016: 18, 69-71 (key to species, description, illustrations). Holotype: 3 , Mexico: Chiapas: 'swift mountain river, $7 \mathrm{mi}$. N of Santa Fe [vic. $17^{\circ} 25^{\prime} 56^{\prime \prime} \mathrm{N}$, $93^{\circ} 02^{`} 05^{\prime \prime} \mathrm{W}$, elev. $\left.\sim 150 \mathrm{~m}\right]$ ’ (JTPC $\rightarrow$ USNM).
Distribution. Neotropical Region: Central America: Mexico: Chiapas (Polhemus \& Polhemus 2016), Veracruz (Polhemus \& Polhemus 2016).

\section{Ochterus strigicollis Horváth, 1913}

Ochterus strigicollis Horváth, 1913: 478-479, Fig. 2 (mislabelled as $O$. marginatus) (description, differential diagnosis, illustration). HoLoTYPE: ㅇ, Israel: 'Wad-es Semakh' (NZSI).

Ochterus strigicollis: PolHemus (1995a): 26 (catalogue).

Distribution. Palaearctic Region: Israel (HoRvÁTH 1913, LiNNAVUORI 1960).

\section{Ochterus stysi}

\section{A. Polhemus \& J. T. Polhemus, 2008}

Ochterus stysi D. A. Polhemus \& J. T. Polhemus, 2008: 282-286 (description, illustrations, key to species for Indian Ocean). НоцотүPE: $\widehat{\partial}$, Mauritius: Black River District: 'stream $1.2 \mathrm{~km}$. S. of Chamarel, 200 m' (BPBM).

Distribution. Afrotropical Region: Mauritius (PoLHEMUS \& Polhemus 2008).

\section{Ochterus surigaoensis Gapud, 1995}

Ochterus surigaoensis Gapud, 1995: 42-43 (description, differential diagnosis, illustrations). HoLotype: $\widehat{\jmath}$, Philippines: Mindanao: 'Mendozana Falls, Bislig, Surigao del Sur' (UPLB).

Ochterus surigaoensis: GAPUD (2003): 107 (checklist); CHEN et al. (2005): 413 (checklist).

Distribution. Oriental Region: Philippines: Mindanao (GAPUD 1995).

Ochterus tenebrosus Nieser, 1975

Ochterus tenebrosus Nieser, 1975: 26, 29-31, Pl. 2c (key to species, description, illustration). HolotyPE: 0 , Suriname: 'Marowijne, road Albinga-Moengo' (RMNH).

Ochterus tenebrosus: HeскMAN (2011): 462 (key to species, illustration); Moreira et al. (2011): 58 (checklist, Brazil); CoRDEIro et al. (2014): 67 (key to species, illustration).

Distribution. Neotropical Region: South America: Brazil: Amazonas (Pereira \& Melo 2007, Moreira et al. 2011, Cordeiro et al. 2014); Suriname (NIESER 1975).

\section{Ochterus thienemanni Jaczewski, 1935}

Ochterus thienemanni Jaczewski, 1935: 480-482 (description, key to species, illustrations). Holotype: $\hat{\jmath}$, Indonesia: Sumatra: 'Mittelsumatra, See von Singkarak, am Wasserfall von Panjingahan, Höhe 362 m' [= Central Sumatra, Singkarak lake, Panjingahan waterfall, $362 \mathrm{~m}$ a.s.1.] (?ZMPA).

Ochterus thienemanni: NIESER \& CHEN (1999): 90-91 (key to species, illustration); Chen et al. (2005): 413 (checklist); Polnemus \& PolmeMUS (2012): 345, 352-355 (key to species, diagnosis, illustrations); Polnemus \& Polnemus (2015): 208-209, 212 (key to species, illustrations).

Distribution. Oriental Region: Indonesia: Bali (JACZEWSKI 1935, Polhemus \& Polhemus 2012), Java (Jaczewski 1935, Polhemus \& Polhemus 2012), Sumatra (Jaczewski 1935, Polhemus \& Polhemus 2012).

Note. The holotype was originally deposited in ZMPA, Warsaw, but it might have been lost during World War II when a large part of Jaczewski's collection was destroyed (see Jaczewski 1949: 107-108, Polhemus \& Polhemus 
2012: 354). Our attempt to contact ZMPA to investigate the whereabouts of the holotype has remained unanswered.

Previous records from New Guinea (Kormilev 1971, BAEHR 1990c, CHEN et al. 2005) represent misidentifications (Polmemus \& Polmemus 2012). The tentative identification of females from Sulawesi by Nieser \& CHEN (1992) was later corrected to O. marginatus by NIESER \& CHEN (1999); the species was listed from Sulawesi by CHEN et al. (2005) in error.

\section{Ochterus sp. not thienemanni Jaczewski, 1935}

Ochterus thienemanni (misidentification): KORMILEV (1971): 437-438 (description, differential diagnosis, illustration); BAEHR (1990b): 451, 453-454, 456-458, 461, 469-470 (key to species, redescription, illustrations); Polhemus \& Polhemus (2012): 354 (clarification of the misidentification)

Distribution. Australian Region: Papua New Guinea (Kormilev 1971, BAEHR 1990b).

\section{Ochterus trichotos Nieser \& Chen, 1999}

Ochterus trichotos Nieser \& Chen, 1999: 90-92-93, 123 (key, description,

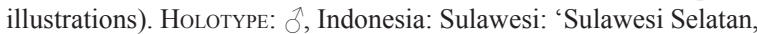
Malino-Manipi, 700 m' (NHMW)

Ochterus trichotos: CHEN et al. (2005): 413 (checklist).

Distribution. Australian Region: Indonesia: Sulawesi (NIESER \& ChEN 1999).

\section{Ochterus unidentatus Nieser \& Chen, 1992}

Ochterus unidentatus Nieser \& Chen 1992: 7-8 (description, differential diagnosis, illustration). Ноцотүре: $\hat{\jmath}$, Ecuador, 'Pichin, Tinalandia' (MZLU).

Ochterus unidentatus: CIANFERONI (2012): 63-64, 67 (key to species for Ecuador, variability, illustrations)

Material examined. ECUADOR: NAPO: $2.7 \mathrm{~km}$ SW of Diaz de Pineda, $0^{\circ} 19^{\prime} 10^{\prime \prime} \mathrm{S} 77^{\circ} 45^{\prime} 33^{\prime \prime} \mathrm{W}, 1560 \mathrm{~m}$ a.s.1., bank of the stony stream, sandy and clay bottom with algae with thin layer of the water, exposed, 1.xii.2006, 1 đ, M. Fikáček \& J. Skuhrovec lgt., P. Kment det. (NMPC). Pastaza: Puyo env., 11.-15.xii.2000, E-38, 1 †, V. Malý lgt., P. Kment det. (NMPC).

Distribution. Neotropical Region: South America: Colombia (MANZANo et al. 1995, PADILLA-GIL 2015); Ecuador (CiAnFERONi 2012).

Note. According to Polhemus \& Polhemus (2016: 44) this species may be a junior synonym of $O$. manni.

\section{Ochterus viridifrons (Champion, 1901)}

Pelogonus viridifrons Champion, 1901: 345-346, Pl. XX: fig. 14 (key, original description, illustration). SyNTYPEs: $\hat{\jmath}$, Guatemala: 'Rio Naranjo’ (BMNH); †, Guatemala: 'San Gerónimo' (BMNH).

Ochterus viridifrons: KIRKALDY \& TORRE BUENO (1909): 179 (catalogue); BARBER (1913): 214 (key to species); Schell (1943a): 32, 35 (key to species, illustration); SCHELl (1943b): 37-38 (original description repeated, differential diagnosis); DraKe (1952): 75 (checklist); Froeschner (1999): 148 (catalogue); HeCKMAN (2011): 459 (key to species, illustration); Cianferoni (2012): 67 (key to species); Herrera Madrigal (2015): 256 (illustration); Polhemus \& Polhemus (2016): $10,15,18,71-73$ (key to species, redescription, illustrations).

Distribution. Neotropical Region: Central America: Costa Rica (Schell 1943b, Herrera Madrigal 2015, Polhemus \& Polhemus 2016); Guatemala (Champion 1910, KirKaLdy \& Torre Bueno 1909, Polhemus \& Polhemus 2016); Honduras (Polhemus \& Polhemus 2016); Mexico (DraKe
1952): Chiapas (Polhemus \& Polhemus 2016), Durango (Nieser \& Chen 1992), Guerrero (Polhemus \& Polhemus 2016), Oaxaca (Polhemus \& Polhemus 2016); Panama (Schell 1943b, Froeschner 1999, Polhemus \& Polhemus 2016). South America: Ecuador (CIANFERONI 2012).

Note. A specimen from Grand Canyon, Arizona, mentioned by Schell (1943b: 38) and Drake (1952) belongs to $O$. rotundus (see Polhemus \& Polhemus 1976: 224).

\section{Ochterus xustos Nieser \& Chen, 1992}

Ochterus xustos Nieser \& Chen, 1992: 12-13 (description, differential diagnosis, illustration). HoLotype: đo, Malaysia: Sabah: 'Jesselton, Sensuran road, mile 25, $4000 \mathrm{ft}$ [= $1219 \mathrm{~m}$ a.s.1.]' (RMNH).

Ochterus xustos: NIESER \& CHEN (1999): 90-91 (key, illustration); CHEN et al. (2005): 413 (checklist).

Distribution. Oriental Region: Malaysia: Sabah (NIESER \& CHEN 1992).

\section{Ochterus zetteli Gapud, 2003}

Ochterus zetteli Gapud, 2003: 103-107 (description, differential diagnosis, illustrations). Holotype: §, Philippines: Palawan: 'Busuanga Island, W. Borac' (UPLB).

Ochterus zetteli: CHEN et al. (2005): 413 (checklist).

Distribution. Oriental Region: Philippines: Palawan: Busuanga Island (GAPUD 2003).

\section{Ochterus sp. near zetteli Gapud, 2003}

Ochterus sp. ( 2 from Philippines: Palawan: Cabayugan River): GAPUD (2003): 106-107 (diagnosis).

Distribution. Oriental Region: Philippines: Palawan (GAPUD 2003).

Note. According to GaPud (2003), this species is highly likely to be new, though closely related to O. zetteli. A male specimen is required in order to dertermine its species status.

\section{Genus Ocyochterus Drake \& Gómez-Menor, 1954}

Ocyochterus Drake \& Gómez-Menor, 1954: 157-158 (original description). Type species: Pelogonus victor Bolívar, 1879, by original designation.

Ocyochterus: NIESER (1975): 26 (key to genera and species); FrOESCHNER (1981): 61 (key to genera); ŠTYS \& JANSSON (1988): 8 (catalogue); YAO et al. (2007): 828, 830 (key to genera, differential table); MAZzUCCONI et al. (2009a): 213 (key to genera); Heckman (2011): 457 (key to genera); YAo et al. (2011): 590, 597-598 (key to genera, phylogeny); Polhemus \& Polhemus (2014): 164-170 (key to genera and species, revision); BARBOSA \& Rodrigues (2015): 174, 176 (key to genera, illustrations); Melo (2015): 344 (key to genera, diagnosis); MoreIRA et al. (2019): 179, 183, 188, 190 (key to genera, illustrations).

Note. Herrera Madrigal (2015) published what was indicated to be the first record of this genus from Colombia, but he was preceded by Polhemus \& Pohemus (2014).

\section{Ocyochterus irmae}

\section{A. Polhemus \& J. T. Polhemus, 2014}

Ocyochterus irmae D. A. Polhemus \& J. T. Polhemus, 2014: 164-170 (key to species, description, illustrations). Ноцотуре: Colombia: 'Valle de Cauca, Farallones de Cali National Park, seeping bedrock faces along upper Rio Ponce, near El Topacio, 1550 m, [vic. $3^{\circ} 19^{\prime} 47^{\prime \prime} \mathrm{N}$ $\left.76^{\circ} 38^{\prime} 13^{\prime \prime} \mathrm{W}\right] '$ (USNM). 
Distribution. Neotropical Region: South America: Colombia (Polnemus \& Polhemus 2014); Ecuador (Polhemus \& Polhemus 2014).

\section{Ocyochterus victor (Bolívar, 1879)}

Pelogonus victor Bolívar 1879: 144 (original description). Holotyp: $\widehat{\jmath}$, Ecuador: 'Pichincha' (IEEM $\rightarrow \mathrm{MNCN})$.

Pelogonus victor: Champion (1901): 344 (list).

Pelogonus Victor: CAMPos (1925): 5 (catalogue).

Ochterus victor: Torre Bueno (1906): 50 (list); Kirkaldy \& Torre BueNO (1909): 179 (catalogue); SCHELl (1943a): 33 (as incertae sedis); SCHELl (1943b): 46 (original description repeated).

Ochterus victors (incorrect subsequent spelling): DRAKE (1952): 75 (checklist).

Ocyochterus victor: DraKe \& Gómez-Menor (1954): 158-159, pl. X: Fig. 1 (new combination, illustrations); FroESCHNER (1981): 61 (catalogue); HeCKMan (2011): 457 (illustrations); CiANFERONI (2012): 67 (key to species for Ecuador); Polmemus \& Polhemus (2014): 164, 168, 170 (key to species, diagnosis, illustrations); Herrera Madrigal (2015): 256 (illustration); Popov \& HeIss (2014a): 187-188 (illustrations).

Pelogonus splendidulus Montandon, 1898: 73-75 (original description, differential diagnosis). Lectotype (designated by DraKe \& Gómez-Menor 1954: 158, as type): đo, Ecuador: 'Nanegal' (MNHN). Junior subjective synonym by DraKe \& Gómez-Menor (1954: 158).

Pelogonus splendidulus: CHAMPION (1901): 344 (list); CAMPOS (1925): 5 (catalogue).

Ochterus splendidulus: ToRre BuEno (1906): 50 (list); KIRKALDY \& TORRE BuEno (1909): 179 (catalogue); Schell (1943a): 32-36 (key to species, translation of original description, differential diagnosis, illustration).

Ochterus spendilulus (incorrect subsequent spelling): DRAKE (1952): 75 (checklist).

Distribution. Neotropical Region: South America: Colombia (Herrera Madrigal 2015); Ecuador (Bolívar 1879, Montandon 1898, Kirkaldy \& Torre Bueno 1909, Drake 1952, Polhemus \& Polhemus 2014).

Note. Montandon (1898) described Pelogonus splendidulus based on an unknown number of specimens. DrAKE \& Gómez-Menor (1954) wrote: 'type, male, in Museum d'Histoire Naturelle, Paris, and a female also labeled type (should be allotype) in British Museum of Natural History, London'. Using the term 'type' for one of the syntypes they used for definition of the taxon, DRAKE \& Gómez-Menor (1954) fulfilled the requirements of the Article 74.5 (ICZN 1999) and their action constitutes a valid lectotype designation.

\section{Fossil record}

\section{Family OCHTERIDAE Kirkaldy, 1906 [1815]}

\section{Genus $\uparrow$ Angulochterus Yao, Zhang \& Ren, 2011}

Angulochterus Yao, Zhang \& Ren, 2011 in YAO et al. (2011): 590, 595-598 (key to genera, original description, phylogeny). Type species: Angulochterus quadrimaculatus Yao, Ren \& Shih, 2011, by original designation.

\section{$\uparrow$ Angulochterus quadrimaculatus Yao, Zhang \& Ren, 2011}

Angulochterus quatrimaculatus Yao, Zhang \& Ren, 2011 in YAO et al. (2011): 595-598 (incorrect original spelling) (original description, illustrations). HoLotyp: $\delta$, CNU-HELB2006644pc (part and counterpart) (CNUB). Type locality and horizon: China: 'Huangbanjigou, Chaomidian Village, Beipiao City, Liaoning Province, Yixian Formation, Early Cretaceous.'
Angulochterus quatlimaculatus (incorrect original spelling): YAO et al. (2011): 590 (cladogram).

Angulochterus quadrimaculatus: SCHUH \& WeIRAUCH (2020): 150-151 (list).

Distribution. China: Liaoning (Yixian Formation: Early Cretaceous) (YAO et al. 2011).

Note. The species name occurs in two different original spellings throughout the text, A. quatrimaculatus seven times throughout the text, A. quatlimaculatus (lapsus calami) twice in the cladogram on page 590. According to their etymology, the 'species name is combination of the Latin quatri- (four) [lapsus calami for quadri-] and maculatus (marking)'. We correct here the spelling of the name as A. quadrimaculatus according to the Article 32.5.1 (ICZN 1999).

\section{Genus $\uparrow$ Floricaudus Yao, Ren \& Shih, 2011}

Floricaudus Yao, Ren \& Shih, 2011 in YAo et al. (2011): 590, 593-594, 597-598 (key to genera, original description, phylogeny). Type species: Floricaudus multilocellus Yao, Ren \& Shih, 2011, by original designation.

\section{$\dagger$ Floricaudus multilocellus Yao, Ren \& Shih, 2011}

Pristinochterus zhangi (misidentification): YAO et al. (2007): 832: fig. 18, 833: fig. 22 (illustration of specimen CNU-HE-LB2006317, see YAO et al. 2011).

Floricaudus multilocellus Yao, Ren \& Shih, 2011 in YAO et al. (2011): 591, 593-594, 598 (original description, illustrations). HoLOTYPE: $\widehat{\delta}$, CNU-HE-LB2008006 (CNUB). Type locality and horizon: China: 'Huangbanjigou, Chaomidian Village, Beipiao City, Liaoning Province, Yixian Formation, Early Cretaceous.'

Floricaudus multilocellus: SchUH \& WeIRAUCH (2020): 150-151 (list).

Distribution. China: Liaoning (Yixian Formation: Early Cretaceous) (YAO et al. 2011).

\section{Genus $\uparrow$ Pristinochterus Yao, Cai \& Ren, 2007}

Pristinochterus Yao, Cai \& Ren, 2007: 827-830 (key to genera, original description, differential table). Type species: Pristinochterus zhangi Yao, Cai \& Ren, 2007, by original designation.

Pristinochterus: YAo et al. (2011): 590, 592-593, 597-598 (key to genera, revision, phylogeny).

\section{$\uparrow$ Pristinochterus ovatus Yao, Zhang \& Ren, 2011}

Pristinochterus ovatus Yao, Zhang \& Ren, 2011 in YAO et al. (2011): 591-592-593, 598 (key to species, original description, illustrations). HolotyPe: $\delta$, CNU-HELB2009096p (part and counterpart) (CNUB). Type locality and horizon: China: 'Huangbanjigou, Chaomidian Village, Beipiao City and Dawangzhangzi Village, Lingyuan City, Liaoning Province, Yixian Formation, Early Cretaceous, 125 Ma.' Pristinochterus ovatus: Schun \& WeIrauch (2020): 152-153 (list).

Distribution. China: Liaoning (Yixian Formation: Early Cretaceous) (YAO et al. 2011).

Note. The exact locality of holotype is not mentioned in the original description.

\section{$\dagger$ Pristinochterus zhangi Yao, Cai \& Ren, 2007}

Pristinochterus zhangi Yao, Cai \& Ren, 2007: 828-830-832 (original description of adult and larva, illustrations). HoLOTYPE: + , CNU-HE-LB2006301-302 (part and counterpart) (CNUB). Type locality and horizon: China: 'Huangbanjigou, Chaomidian Village, Beipiao City and Dawangzhangzi Village, Lingyuan City, Liaoning Province, Yixian Formation, Late Jurassic; Balihan Village, Ningcheng County, Chifeng City, Inner Mongolia Autonomous Region, China, Jiufotang Formation, Early Cretaceous.' 
Pristinochterus zhangi: YAO et al. (2010): 142-143 (diagnosis, illustration); YAO et al. (2011): 591-592, 598 (key to species); ScHuH \& WeIRAUCH (2020): 152-153 (list).

Distribution. China: Liaoning (Yixian Formation: Late Jurassic to Early Cretaceous) (YaO et al. 2007, 2010), Inner Mongolia (Jiufotang Formation: Early Creataceous) (YAO et al. 2007, 2010).

Note. Exact locality of holotype is not mentioned in the original description.

\section{Genus $\uparrow$ Riegerochterus Popov \& Heiss, 2014}

Riegerochterus Popov \& Heiss, 2014a: 186-187 (description, differential diagnosis). Type species: Riegerochterus baehri Popov \& Heiss, 2014a, by original designation.

\section{$\dagger$ Riegerochterus baehri Popov \& Heiss, 2014}

Riegerochterus baehri Popov \& Heiss, 2014a: 187-188 (description, differential diagnosis, illustrations). Ноцотуре: $\hat{\sigma}$, 'in a piece of irregular shaped Dominican Amber $(17 \times 17 \times 13 \mathrm{~mm})$, Type. Kat. Nr. Do-4667-B' (SMNS).

Riegerochterus baehri: SCHUH \& WEIRAUCH (2020): 152-153 (list).

Distribution. Dominican amber (?Early to Middle Miocene) (Popov \& Heiss 2014a).

\section{Ochteridae incertae sedis}

\section{Genus $\uparrow$ Yuripachys nom. nov.}

Meropachys Popov, 1986: 78-79 (original description). Type species: Meropachys dubius Popov, 1986, by original designation. Junior homonym of Meropachys Burmeister, 1835 (Heteroptera: Coreidae).

Meropachys: Popov \& Heiss (2014a): 186 (systematic placement).

Note. The generic name Meropachys Popov, 1986 is a junior homonym of Meropachys Burmeister, 1835, a valid genus of the family Coreidae (Hemiptera: Heteroptera), including two valid species from South America (PACKAUSKAS 2010, ANONymus 2019). As there is no junior synonym available as a replacement name, we propose here a new substitute name, Yuripachys nom. nov., to replace Meropachys Popov, 1986. The new name is dedicated to Yuri A. Popov (1936-2016), a great Russian specialist in heteropteran palaeontology, being composed of his given name and the Greek adjective pachys ( $\pi \alpha \chi v$ $\varsigma)$, meaning for example thick, large, stout, coarse, fat, or great. The gender is masculine; the grammatical stem is Yuripache-.

\section{$\dagger$ Yuripachys dubius (Popov, 1986) comb. nov.}

Meropachys dubius Popov, 1986: 75: fig. 45, 79, Pl. V: fig. 5 (original description, illustrations). НоLOTYPE: + , positive part, body dorsally without head and fore legs, 'ПИН, N 3152/806', 'Myangad, 221/17' (PIN).

Meropachys dubius: Popov \& HeIss (2014a): 186 (systematic placement); Schun \& Weirauch (2020): 180-181 (listed in Pachymeridiidae).

Distribution. West Mongolia (Gurban-Eren Formation: Early Cretaceous) (Popov 1986).

Note. The species was originally placed in the Mesozoic lygaeoid family Pachymeridiidae (Popov 1986), but according to Popov \& Heiss (2014a) it more probably belongs to Ochteridae; this change was overlooked by $\mathrm{S}_{\mathrm{CHUH}} \&$ WEIRAUCH (2020).
Family $\uparrow$ PROPREOCORIDAE Popov, Dolling \& Whalley, 1994, stat. nov.

'Leptopodomorpha. Family nov. 2': WhALLEY (1985): 145.

Propreocorinae Popov, Dolling \& Whalley, 1994: 335-336 (original description). Type genus: Propreocoris Popov, Dolling \& Whalley, 1994.

Propreocorinae: SHCHERBAKOv \& PoPOv (2002): 151 (systematic placement); Schuh \& Weirauch (2020): 152-153 (list).

Properocorinae (incorrect subsequent spelling): SchuH \& WeIRauch (2020): 139 (list).

Proprepocorinae (incorrect subsequent spelling): SchuH \& WeIRAuch (2020): 140 (list).

Note. According to Grimaldi \& Engel (2005) and Yao et al. (2011) Propreocoris represents a common ancestor of the Ochteridae and Gelastocoridae. As long as Propreocorinae are no longer included in Ochteridae, we formally raise this taxon to family rank.

\section{Genus $\uparrow$ Propreocoris \\ Popov, Dolling \& Whalley, 1994}

'Gen. et sp. nov. 2A': Whalley (1985): 144: fig. 41, 145 (diagnosis, illustration).

Propreocoris Popov, Dolling \& Whalley, 1994: 317, 333, 336-337 (original description). Type species: Propreocoris maculatus Popov, Dolling \& Whalley, 1994, by original designation.

Propreocoris: GRIMALDI \& ENGEL (2005): 320-321 (systematic placement); YAO et al. (2007): 828, 830 (key to genera, differential table); YAO et al. (2011): 591-592 (systematic placement).

\section{$\uparrow$ Propreocoris maculatus \\ Popov, Dolling \& Whalley, 1994}

'Gen. et sp. nov. 2A': Whalley (1985): 144: fig. 41, 145 (diagnosis, illustration).

Propreocoris maculatus Popov, Dolling \& Whalley, 1994: 317, 333, 336 (original description, illustrations). Ноцотуре: ?sex (part and counterpart), In.59152, United Kingdom: England: 'The Woodstone, Black Ven, Charmouth, Dorset; Lower Lias' (BMNH: coll. Jackson). Propreocoris maculatus: SchuH \& WeIRAuch (2020): 139, 152-153 (list).

Distribution. United Kingdom: England: Dorset (Charmouth fauna: Early Jurassic (Lower Lias)) (Popov et al. 1994). Note. The fossil is represented by a poorly preserved partial body, lacking legs and head.

\section{Ochteroidea incertae sedis}

\section{Genus $\uparrow$ Grimaldinia Popov \& Heiss, 2014}

Grimaldinia Popov \& Heiss, 2014b: 445-448 (original description). Type species: Grimaldinia pronotalis Popov \& Heiss, 2014b, by original designation.

Grimaldinia: SCHUH \& WeIRAUCH (2020): 139, 152-153 (revised systematic placement).

Note. Popov \& Heiss (2014b) described the genus Grimaldinia in family Leptopodidae, subfamily Leptosaldinae. Schun \& Weirauch (2020) concluded that the fossil belongs to Ochteroidea based on the short antennae that are held at rest and inserted under the eyes and the anastomosing veins forming numerous membrane cells, characters not present in any known Leptopodomorpha.

\section{$\dagger$ Grimaldinia pronotalis Popov \& Heiss, 2014}

Grimaldinia pronotalis Popov \& Heiss, 2014b: 446-449 (original description, illustrations). HoLOTYPE: ?sex, sub-macropterous (BUB-LEP-1), Burmese Amber (EHIA). 
Grimaldinia pronotalis: SchUH \& WeIRAUCH (2020): 139, 152-153 (revised systematic placement).

Distribution. Myanmar: Kachin (Burmese Amber: Mid-Cretaceous, Late Albian-Early Cenomanian, ca. 97-110 mya) (Popov \& HeIss 2014b).

\section{Genus $\uparrow$ Heterochterus Evans, 1971}

Heterochterus Evans, 1971: 149-150 (original description). Type species: Heterochterus timmsii Evans, 1971, by original designation. Heterochterus: JelL (2004): 65 (redescription, illustrations).

Note. Evans (1971) placed this isolated wing in Heteroptera and noted that it is closely resembling the wing of Ochterus marginatus. This fossil has not been mentioned by any of the previous papers focused on fossil Ochteridae (cf. PoPOV et al. 2004; YAo et al. 2007, 2011; Popov \& Heiss 2014a), except Shcherbakov \& Popov (2002: 150) who listed it as 'possible Ochteroidea'.

\section{$\dagger$ Heterochterus timmsii Evans, 1971}

Heterochterus timmsii Evans, 1971: 149-150 (original description, illustration). Ноцотуре: ?sex, wing, Australia: Queensland: 'Mt Crosby, coll. B. V. Timms, presented by Department of Entomology, University of Queensland, Q.M. F6473' (QMBA).

Heterochterus timmsii: JELL (2004): 65 (redescription, illustrations).

Distribution. Australia: Queensland (Ipswich Coal Measures: Late Triassic) (Evans 1971, JELL 2004).

\section{Discussion}

The first described species of velvety shore bugs was the widely distributed Acanthia marginata Latreille, 1804, discovered in southern France, and soon after accommodated in its own new genus, Ochterus Latreille, 1807, and new family, Pelogonida Leach, 1815 (based on an unnecessary new replacement name Pelogonus Latreille, 1809). Additional two taxa were described by GuÉrin-MéNeville (1843), Pelogonus indicus (junior synonym of $O$. marginatus marginatus) from India, and the first American species, Pelogonus perboscii from Mexico. Until the end of 19 th century, altogether eleven species of Ochteridae were described, seven of which still remain valid. Among them were also two species which were in the future designated as types of new genera, Ocyochterus Drake \& Gómez-Menor, 1954 (Pelogonus victor Bolívar, 1879) and Megochterus Jaczewski, 1934 (Pelogonus nasutus Montandon, 1898). Between 1901 and 1970, 17 additional species of Ochteridae were described, mostly from the American continent where important contributions were published by Champion (1901), Barber (1913), Hungerford (1927), and especially the revision by SCHELL (1943a,b). In the Old World, only five species were described in this period (HoRvÁth 1913; JACZEWSKI 1934, 1935, 1938; MANCINI 1939). Singh-PRUTHI (1925) first described and illustrated male genitalia including the right paramere of Ochterus marginatus, a character soon applied in ochterid taxonomy by Hungerford (1927) and JACZEWSKI (1934). Big progress in describing new Ochteridae started in 1970s with three revisional papers by Nicolas A. Kormilev for Australian, Oriental and Afrotropical taxa (KoRMILEV $1971,1972,1973)$, largely based on the structure of the right paramere. Soon followed the papers of NIESER (1975) (Suriname), Polhemus \& Polhemus (1976) (SW USA), Rieger (1977) (Australia, Philippines), and GAPUD \& SAN VALENTIN (1977) (Philippines). Victor P. Gapud continued his work on the fauna of Philippines also in the following three decades (GAPUD 1981, 1995, 2003). In the same time,

Table 1. Number of species-group taxa of Ochteridae described over the time (valid recent taxa only).

\begin{tabular}{lcc}
\hline Decade & $\begin{array}{l}\text { Number } \\
\text { per decade }\end{array}$ & $\begin{array}{l}\text { Cumulative } \\
\text { number }\end{array}$ \\
\hline $1800 \mathrm{~s}$ & 1 & 1 \\
\hline $1840 \mathrm{~s}$ & 1 & 2 \\
\hline $1850 \mathrm{~s}$ & 1 & 3 \\
\hline $1860 \mathrm{~s}$ & 1 & 4 \\
\hline $1870 \mathrm{~s}$ & 2 & 6 \\
\hline $1890 \mathrm{~s}$ & 1 & 7 \\
\hline
\end{tabular}

\begin{tabular}{lcc}
\hline Decade & $\begin{array}{l}\text { Number } \\
\text { per decade }\end{array}$ & $\begin{array}{l}\text { Cumulative } \\
\text { number }\end{array}$ \\
\hline $1900 \mathrm{~s}$ & 3 & 10 \\
\hline $1910 \mathrm{~s}$ & 2 & 12 \\
\hline $1920 \mathrm{~s}$ & 2 & 14 \\
\hline $1930 \mathrm{~s}$ & 4 & 18 \\
\hline $1940 \mathrm{~s}$ & 4 & 22 \\
\hline $1950 \mathrm{~s}$ & 2 & 24 \\
\hline
\end{tabular}

\begin{tabular}{lcc}
\hline Decade & $\begin{array}{l}\text { Number } \\
\text { per decade }\end{array}$ & $\begin{array}{l}\text { Cumulative } \\
\text { number }\end{array}$ \\
\hline $1970 \mathrm{~s}$ & 16 & 40 \\
\hline $1980 \mathrm{~s}$ & 6 & 46 \\
\hline $1990 \mathrm{~s}$ & 21 & 67 \\
\hline $2000 \mathrm{~s}$ & 3 & 70 \\
\hline $2010 \mathrm{~s}$ & 17 & 87 \\
\hline $2020 \mathrm{~s}$ & 1 & 88 \\
\hline
\end{tabular}

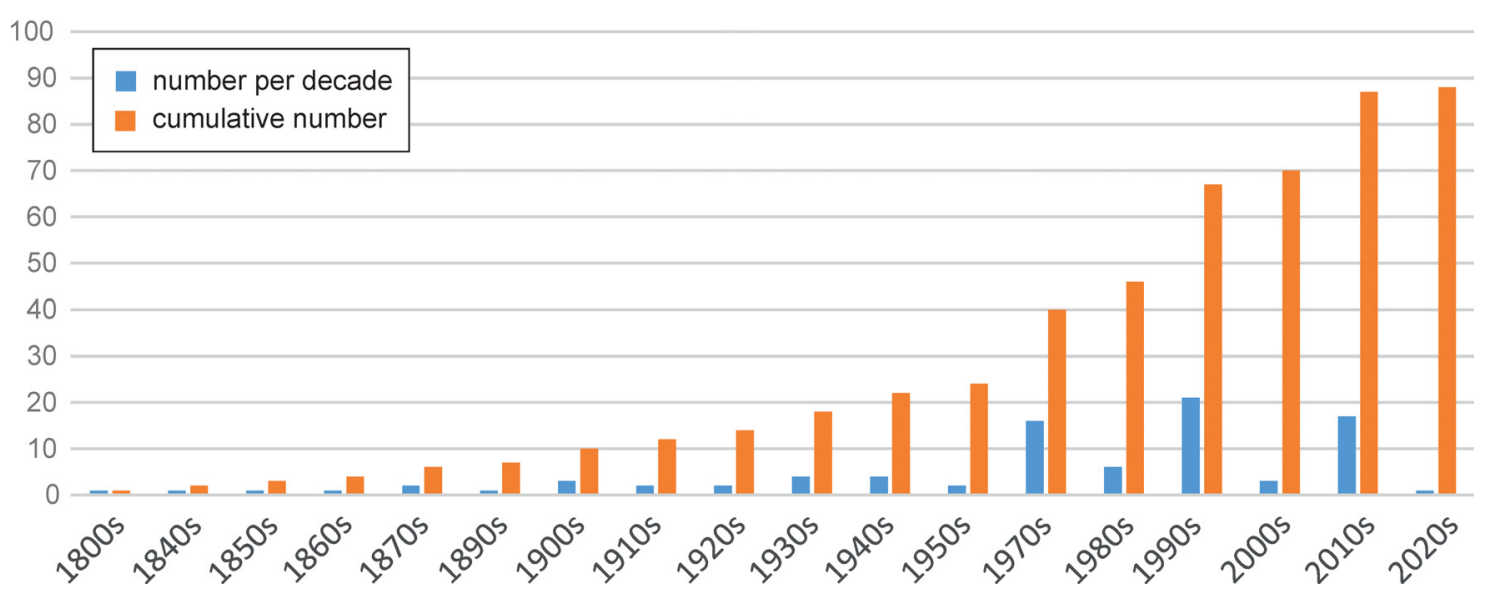

Fig. 25. Progress in describing new species-group taxa of Ochteridae (valid recent taxa only). 
Table 2. Distribution of recent Ochteridae in particular zoogeographical regions. In the table Yemen is treated as part of Afrotropical Region, China: Yunnan as part of Oriental Region, and Mexico: Sonora as part of Nearctic Region. We use Wallace line as the boundary between Oriental and Australian Region, and boundary between Thailand and Malaysia as boundary between Continental Asia and Sundaland. New Guinea includes also the Bismarck Archipelago.

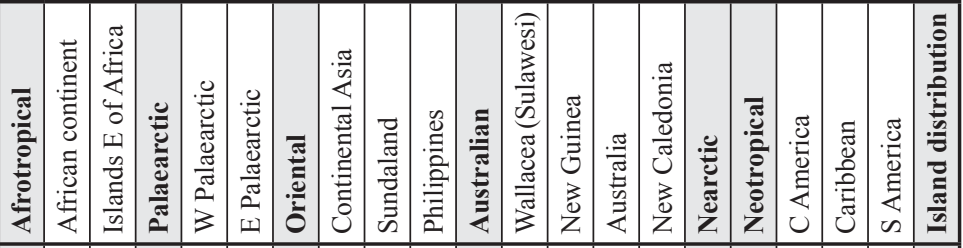

Megochterus nasutus (Montandon, 1898)

M. occidentalis Baehr, 1990

Ochterus africanus Jaczewski, 1938

O. caffer (Stål, 1855)

O. minor Kormilev, 1973

O. piliferus Kormilev, 1973

O. papaceki Kment \& Carapezza, 2020

O. seychellensis D.A. Polhemus, 1992

O. stysi D.A. Polhemus \& J.T. Polhemus, 2008

O. marginatus marginatus (Latreille, 1804)

O. strigicollis Horváth, 1913

O. breviculus Nieser \& Chen, 1992

O. feae Mancini, 1939

O. nicobarensis Chandra \& Jehamalar, 2012

O. signatus D.A.Polhemus \& J.T.Polhemus, 2012

O. bruneiensis Zettel \& Lane, 2010

O. pseudomarginatus D.A. Polhemus \& J.T. Polhemus, 2016 O. singaporensis D.A. Polhemus \& J.T. Polhemus, 2016

O. thienemanni Jaczewski, 1935

O. xustos Nieser \& Chen, 1992

O. baltazarae Gapud \& San Valentin, 1977

O. barrosoi Gapud, 1981

O. luzonicus Gapud, 2003

O. magnificus Gapud \& San Valentin, 1977

O. magnus Gapud \& San Valentin, 1977

O. marginatus insularis Rieger, 1977

O. pardalos Nieser \& Chen, 1999

O. philippinensis Kormilev, 1971

O. polhemusi Gapud, 1981

O. surigaoensis Gapud, 1995

O. zetteli Gapud, 2003

O. noualhieri Baehr, 1990

O. grandiusculus Nieser \& Chen, 1992

O. homorfos Nieser \& Chen, 1999

O. trichotos Nieser \& Chen, 1999

O. alticola Baehr, 1990

O. cheesmannae Baehr, 1990

O. gressitti Kormilev, 1971

O. jaczewskii Kormilev, 1971

O. kokodae Baehr, 1990

O. latior Baehr, 1990

O. louisiadae Baehr, 1990

O. nigrinus Baehr, 1990

O. papuasicus Kormilev, 1972

O. paucistriatus Baehr, 1990

O. australicus Jaczewski, 1934

O. atridermis Baehr, 1989

O. bacchusi Baehr, 1990

O. baehri baehri Rieger, 1977

O. baehri riegeri Baehr, 1989

O. brachysoma Rieger, 1977

O. eurythorax Baehr, 1989

O. monteithorum Baehr, 1990

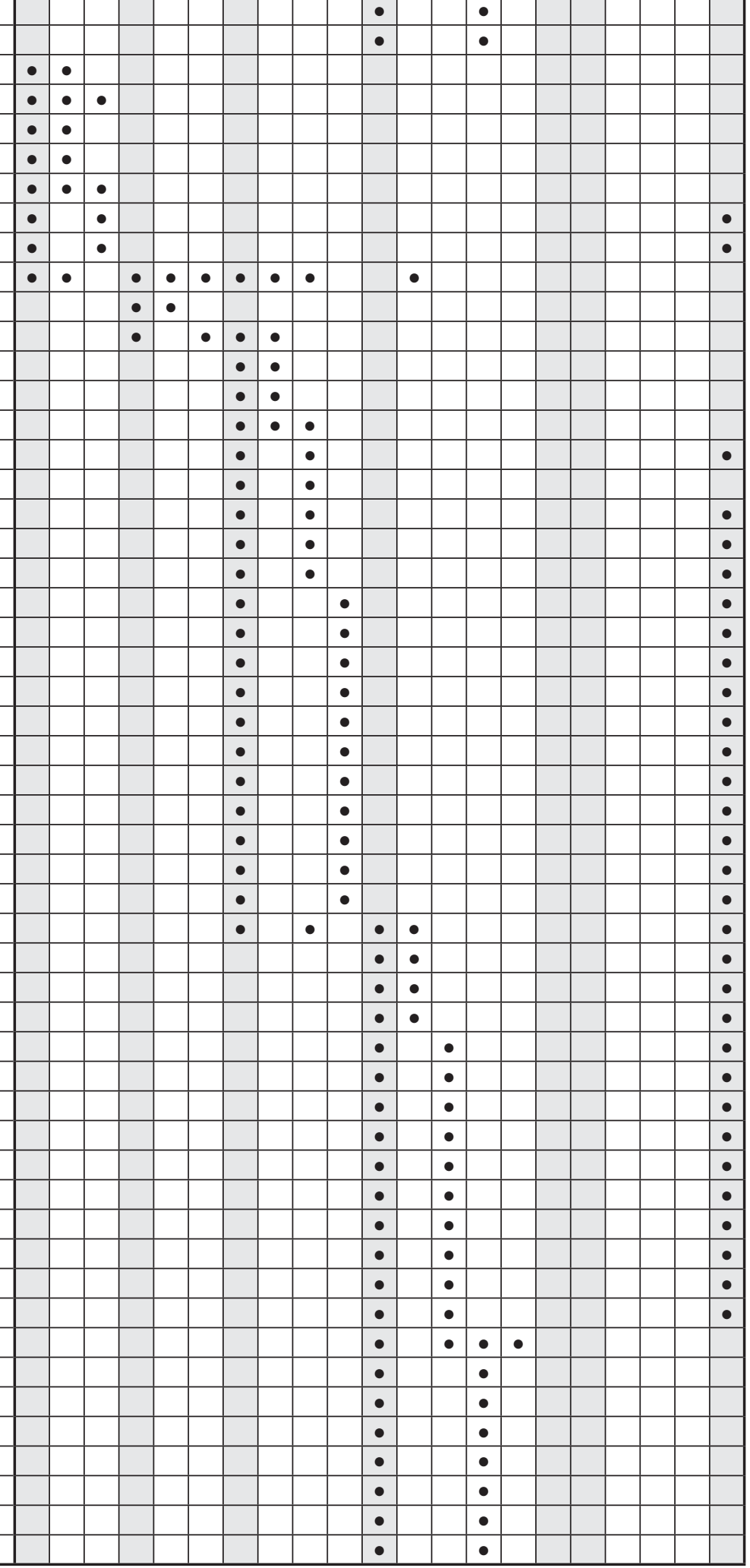


Table 2. Continued.

O. occidentalis Baehr, 1990

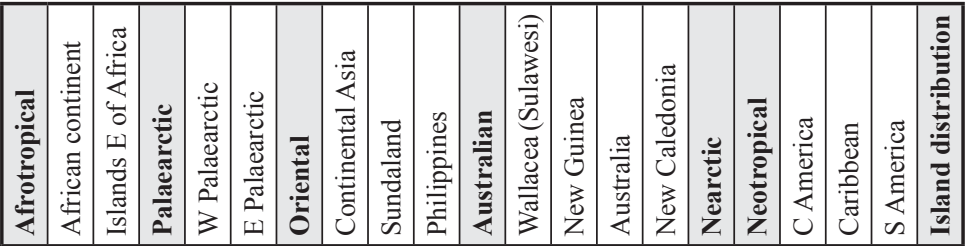

O. secundus secundus Kormilev, 1971

O. secundus pseudosecundus Baehr, 1989

O. dufourii (Montrouzier, 1864)

O. americanus (Uhler, 1876)

O. banksi Barber, 1913

O. barberi Schell, 1943

O. rotundus J.T. Polhemus \& M.S. Polhemus, 1976

O. perbosci (Guérin-Méneville, 1843)

O. acutangulus (Champion, 1901)

O. chiapensis D.A. Polhemus \& J.T. Polhemus, 2016

O. costaricensis D.A. Polhemus \& J.T. Polhemus, 2016

O. explanatus D.A. Polhemus \& J.T. Polhemus, 2016

O. mexicanus D.A. Polhemus \& J.T. Polhemus, 2016

O. obscurus D.A. Polhemus \& J.T. Polhemus, 2016

O. ovatus D.A. Polhemus \& J.T. Polhemus, 2016

O. panamensis D.A. Polhemus \& J.T. Polhemus, 2016

O. pseudorotundus D.A. Polhemus \& J.T. Polhemus, 2016

O. schellae Drake, 1952

O. shepardi D.A. Polhemus \& J.T. Polhemus, 2016

O. smaragdinus D.A. Polhemus \& J.T. Polhemus, 2016

O. hungerfordi Schell, 1943

O. aeneifrons aeneifrons (Champion, 1901)

O. manni Hungerford, 1927

O. viridifrons (Champion, 1901)

O. aeneifrons surinamensis Nieser, 1975

O. bidentatus Schell, 1943

O. brunneus Hungerford, 1927

O. foersteri Kormilev \& De Carlo, 1952

O. parvus Schell, 1943

O. santosi Cordeiro \& Moreira, 2014

O. tenebrosus Nieser, 1975

O. unidentatus Nieser \& Chen, 1992

Ocyochterus irmae D.A. Polhemus \& J.T. Polhemus, 2014

O. victor (Bolívar, 1879)

Number of species in the region

\begin{tabular}{|c|c|c|c|c|c|c|c|c|c|c|c|c|c|c|c|c|c|c|c|c|}
\hline & & & & & & & & & & - & & & $\bullet$ & & & & & & & \\
\hline & & & & & & & & & & $\bullet$ & & & $\bullet$ & & & & & & & \\
\hline & & & & & & & & & & $\bullet$ & & & $\bullet$ & & & & & & & \\
\hline & & & & & & & & & & $\bullet$ & & & & $\bullet$ & & & & & & • \\
\hline & & & & & & & & & & & & & & & $\bullet$ & & & & & \\
\hline & & & & & & & & & & & & & & & $\bullet$ & & & & & \\
\hline & & & & & & & & & & & & & & & $\bullet$ & & & & & \\
\hline & & & & & & & & & & & & & & & $\bullet$ & $\bullet$ & $\bullet$ & & & \\
\hline & & & & & & & & & & & & & & & - & - & $\bullet$ & $\bullet$ & $\bullet$ & \\
\hline & & & & & & & & & & & & & & & & $\bullet$ & $\bullet$ & & & \\
\hline & & & & & & & & & & & & & & & & $\bullet$ & $\bullet$ & & & \\
\hline & & & & & & & & & & & & & & & & $\bullet$ & $\bullet$ & & & \\
\hline & & & & & & & & & & & & & & & & • & $\bullet$ & & & \\
\hline & & & & & & & & & & & & & & & & $\bullet$ & $\bullet$ & & & \\
\hline & & & & & & & & & & & & & & & & $\bullet$ & $\bullet$ & & & \\
\hline & & & & & & & & & & & & & & & & $\bullet$ & $\bullet$ & & & \\
\hline & & & & & & & & & & & & & & & & $\bullet$ & $\bullet$ & & & \\
\hline & & & & & & & & & & & & & & & & $\bullet$ & $\bullet$ & & & \\
\hline & & & & & & & & & & & & & & & & $\bullet$ & $\bullet$ & & & \\
\hline & & & & & & & & & & & & & & & & $\bullet$ & $\bullet$ & & & \\
\hline & & & & & & & & & & & & & & & & $\bullet$ & $\bullet$ & & & \\
\hline & & & & & & & & & & & & & & & & $\bullet$ & . & $\bullet$ & & $\bullet$ \\
\hline & & & & & & & & & & & & & & & & $\bullet$ & $\bullet$ & $\bullet$ & • & \\
\hline & & & & & & & & & & & & & & & & $\bullet$ & $\bullet$ & & $\bullet$ & \\
\hline & & & & & & & & & & & & & & & & $\bullet$ & $\bullet$ & & $\bullet$ & \\
\hline & & & & & & & & & & & & & & & & $\bullet$ & & & $\bullet$ & \\
\hline & & & & & & & & & & & & & & & & $\bullet$ & & & $\bullet$ & \\
\hline & & & & & & & & & & & & & & & & $\bullet$ & & & $\bullet$ & \\
\hline & & & & & & & & & & & & & & & & $\bullet$ & & & $\bullet$ & \\
\hline & & & & & & & & & & & & & & & & $\bullet$ & & & $\bullet$ & \\
\hline & & & & & & & & & & & & & & & & $\bullet$ & & & $\bullet$ & \\
\hline & & & & & & & & & & & & & & & & $\bullet$ & & & $\bullet$ & \\
\hline & & & & & & & & & & & & & & & & $\bullet$ & & & $\bullet$ & \\
\hline & & & & & & & & & & & & & & & & $\bullet$ & & & $\bullet$ & \\
\hline & & & & & & & & & & & & & & & & $\bullet$ & & & $\bullet$ & \\
\hline 8 & 6 & 4 & 3 & 2 & 2 & 22 & 5 & 8 & 11 & 29 & 5 & 11 & 13 & 2 & 5 & 28 & 17 & 3 & 14 & 33 \\
\hline
\end{tabular}

Table 3. Stratigraphic and geographic distribution of fossil Ochteroidea.

\begin{tabular}{|c|c|c|c|c|c|c|c|c|c|c|c|c|c|}
\hline & & 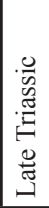 & 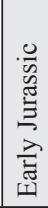 & 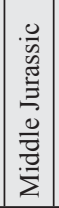 & 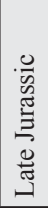 & 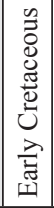 & 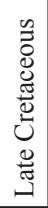 & 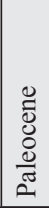 & 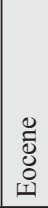 & 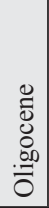 & $\begin{array}{l}\stackrel{Ð}{0} \\
\stackrel{\Xi}{0} \\
\stackrel{0}{\Sigma}\end{array}$ & 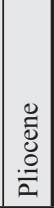 & \\
\hline $\begin{array}{l}\text { Ochteroidea } \\
\text { incertae sedis }\end{array}$ & Heterochterus timmsii Evans, 1971 & $\bullet$ & & & & & & & & & & & Australia \\
\hline Propreocoridae & Propreocoris maculatus Popov, Dolling \& Whalley, 1994 & & $\bullet$ & & & & & & & & & & England \\
\hline $\begin{array}{l}\text { Ochteridae } \\
\text { incertae sedis }\end{array}$ & Yuripachys dubius (Popov, 1986) & & & & & $\bullet$ & & & & & & & Mongolia \\
\hline Ochteridae & Pristinochterus zhangi Yao, Cai \& Ren, 2007 & & & & $\bullet$ & $\bullet$ & & & & & & & China \\
\hline Ochteridae & Angulochterus quadrimaculatus Yao, Zhang \& Ren, 2011 & & & & & $\bullet$ & & & & & & & China \\
\hline Ochteridae & Floricaudus multilocellus Yao, Ren \& Shih, 2011 & & & & & $\bullet$ & & & & & & & China \\
\hline Ochteridae & Pristinochterus ovatus Yao, Zhang \& Ren, 2011 & & & & & $\bullet$ & & & & & & & China \\
\hline Ochteridae & Riegerochterus baehri Popov \& Heiss, 2014 & & & & & & & & & & $\bullet$ & & Dominican amber \\
\hline $\begin{array}{l}\text { Ochteroidea } \\
\text { incertae sedis }\end{array}$ & Grimaldinia pronotalis Popov \& Heiss, 2004 & & & & & & $\bullet$ & & & & & & Burmese amber \\
\hline
\end{tabular}


Martin Baehr, a renowned German coleopterist specialised in Carabidae (Coleoptera), revised Ochteridae of the Australian Region in a series of four papers (BAEHR 1989, 1990a,b,c). In 1990s, Nico Nieser and Ping-ping Chen described seven additional species mostly from Malesia, but also from southern China and South America (NIESER \& CHEN 1992, 1999). Finally, Dan A. Polhemus started describing new Ochteridae with Ochterus seychellensis in 1992 (Polhemus 1992a), and continued, together with his father John T. Polhemus, with series of revisional papers on Ochterus of Mauritius (Polhemus \& Polhemus 2008), Malaysia and the Oriental part of Indonesia (2012, 2015), North and Central America (Polhemus \& Polhemus 2016), as well as a revision of the genus Ocyochterus with the description of one new species (Polhemus \& Polhemus 2014). Since 1971, 64 species of Ochteridae were described, nearly three times the number described before this date (see Table 1 and Fig. 25). The most prolific authors were D. A. and J. T. Polhemus, who described together 17 species of Ochteridae, followed by M. Baehr with 16 species and subspecies, N. Nieser with 9 species, and N. A. Kormilev and V. P. Gapud each with 8 species (cf. Table 2). The species inventory of Ochteridae is certainly far from complete, as it is documented by mentions of a probable new species of Ochterus from Palawan (GAPUD 2003) and several undescribed species from New Guinea (Polnemus \& Polhemus 1998, 2012), and Oriental Region, as well as due to the probable polyphyly of $O$. marginatus (CHEN et al. 2005). Also our unpublished results suggest existence of additional undescribed species, especially in the Oriental Region, the Afrotropical Region and Madagascar.

The extant members of the family Ochteridae are distributed in the tropical and subtropical regions of all continents. The present lack of published records from Lesser Sunda and Maluku Islands (Indonesia) is probably due to sampling bias, as we have at hand unidentified material from Maluku, superficially resembling $O$. nicobarensis (ZJPC). Further Ochteridae inhabit temperate zones of Australia and Tasmania (Andersen \& WeIr 2004), East Palearctic (north to the northernmost Japanese island, Hokkaido, and to Kunashir, the southernmost island of Kuriles) (Polhemus 1995a, Kanyukova 2006, Hayashi et al. 2016, Hayashi \& MiуAмото 2018) and Eastern Nearctic Region (north to Massachusetts, Michigan, Minnesota and Ontario (Polhemus \& Polhemus 1988a, 2016; Maw et al. 2000). On the contrary, Ochteridae are missing in temperate Europe, continental regions of Central Asia (Polhemus 1995a, KanYUKOva 2006), from the Appalachian Mountains and the Ozark Plateau in the eastern part, as well as from most of the western part of USA (Polhemus \& Polhemus 1988a, 2016), from New Zealand, and from temperate and subtropical South America (the most southern species being $O$. foersteri reaching the Misiones province of Argentina - Melo 2015, Bachmann \& Mazzucconi 2017). The zoogeographical pattern of Gelastocoridae resembles that of Ochteridae, with three exceptions: i) Gelastocoridae in South America occur further south, into central Chile; ii) in the Eastern Palaearctic they extend northward only to central China and the southern Japanese islands of Kyushu and Shikoku; and iii) they are completely absent in the Western Palaearctic (except one record from coast of Sinai, Egypt) (e.g., Todd 1955, 1961; Polhemus \& Polhemus 1988b; Polhemus 1995b; Andersen \& Weir 2004; Chen et al. 2005; KMENT \& JindRA 2008; FAÚNDEZ \& AsHWORTH 2015; Faúndez \& Carvajal 2017; Nieser et al. 2020).

Concerning the biodiversity pattern, we can recognize two distinct biodiversity centres, the Australasian Region with 29 species-group taxa and the Neotropical Region with 28 species-group taxa. Within the Neotropical Region, most of these taxa (i.e. 17) are known from Central America, while only 14 species and subspecies are known from South America (see Table 2). This situation may be influenced by the existence of the recent excellent revision of Central American fauna by Polhemus \& Polhemus (2016) and future research will elucidate the precise number of species actually occurring in South America. In the Caribbean, so far only one endemic species is known $(O$. hungerfordi from Cuba) and two widely distributed species shared with the continent (O. aeneifrons aeneifrons and $O$. perbosci), which is in contrast with high number of island endemics occurring in Malesia and islands of the Indian Ocean. In the Australian Region, 5 species occur in Sulawesi (including the widely distributed $O$. marginatus marginatus and $O$. noualhieri shared with Sundaland), 11 species in New Guinea, Vanuatu and Solomon Islands, 13 species and subspecies in Australia, and 2 species in New Caledonia (one of them endemic). The Oriental Region seems also species rich with 22 species-group taxa, but only 5 species are so far known in its western (mostly continental) part north of the border of Malaysia; 8 species occur in Sundaland and 11 species and subspecies are confined to the Philippines. The Afrotropical Region harbours 8 species, 6 of them occurring on the continent and 4 on the adjacent islands; $O$. caffer and $O$. papaceki sp. nov. occur in both the African mainland and islands (Madagascar and Socotra, respectively), while Mauritius and Seychelles each harbour a single endemic species. The fauna of the Nearctic Region includes five species (with $O$. rotundus and $O$. perbosci confined to its southernmost regions and shared with Neotropical Region) (PoLHEMus $\&$ Polmemus 2016). The least species rich is fauna of the Palaearctic Region, including only three described species, the widely distributed $O$. marginatus marginatus, $O$. strigicollis endemic to Israel, and $O$. breviculus confined to the transitional zone between the Palaearctic and the Oriental Region in Yunnan and Xizang (Tibet) in southern China (Nieser \& Chen 1992, Polhemus 1995a). In alternative zoogeographical regionalisation, Malesia (including the Malay Peninsula, Sundaland, Philippines, Wallacea, New Guinea and Solomon Islands), would harbour 33 species and subspecies - see Chen et al. 2005, Zettel \& LANE 2010. Polhemus \& Polhemus 2012). In contrast to the New World, we must stress the importance of island endemism for the species diversity in Old World Ochterus - among 55 Old World species, 33 (60\%) of them are confined to islands. Interestingly, also Gelastocoridae also share a similar pattern of species richness, with centres of biodiversity in Neotropical and Australian Region; the Oriental Region 
harbours only 9 species, 3 of them extending to Eastern Palaearctic, the Afrotropical Region has only 2 species, one of them marginally reaching the Western Palaearctic; 8 species-group taxa occur in Nearctic Region, though most of them are confined to its southernmost areas, Florida and along the Mexican border (e.g., TodD 1955, 1961; Polhemus \& Polhemus 1988b; Polhemus 1995b; Andersen \& WeIR 2004; Chen et al. 2005; KMENT \& JiNDRA 2008; FAÚNDEZ \& AshWORTH 2015; NiESER et al. 2020).

The phylogenetic relationships within Ochteridae were addressed by MAHNER (1993) and summarized as follows: (Megochterus + (Ocyochterus + (American Ochterus + Old World Ochterus))). The monophyly of the Old World Ochterus is well supported by the apomorphic structure of the right paramere, provided with a rounded apical cap and two subapical appendages; however, the monophyly of American Ochterus with a simple right paramere remains questionable, as their paraphyly towards the Old World clade (Ochterus s. str.) is also possible (MAHNER 1993). The first cladistic analysis of relationships among genera of Ochteridae was performed by YAO et al. (2011), who included the three recent genera and three fossil genera from Late Jurassic and Early Cretaceous of China, suggesting the following relationships: $((\dagger$ Floricaudus $+\dagger$ Pristinochterus $)+(\uparrow$ Angulochterus $+($ Megochterus $+($ Ochterus + Ocyochterus)))). For review of the fossil taxa assigned to Ochteridae and Ochteroidea stem group see Table 3. On the other hand, relationships among species-group taxa within the diverse Ochterus have never been rigorously tested. Considering the morphological homogeneity of this group, the use of molecular phylogenetic methods will be necessary.

\section{Acknowledgements}

We thank the late Pavel Štys (Charles University, Prague, Czech Republic) for sharing his manuscript notes on the names and nomenclatural acts made by MAHNER (1993). We are further indebted to Elena Kanyukova (Far Eastern Federal University, Zoological Museum, Vladivostok, Russia) and Kazutaka Yamada (Tokushima Prefectural Museum, Tokushima, Japan) for their help in tracing references for the fauna of the former Soviet Union and Japan, respectively. Thomas J. Henry (USNM), Nico Nieser (Tiel, the Netherlands), and Christian Rieger (Nürtingen, Germany) kindly provided us with information on type depositories of particular species. We are also obliged to Viktor Hartung (ZMUH) and Roberto Poggi (MCSN) for taking photographs of the holotypes of Ochterus africanus and O. feae, respectively. Fabio Cianferoni (Natural History Museum, University of Florence, Italy), Leonidas R. Davranoglou (Department of Zoology, University of Oxford, United Kingdom), Dan A. Polhemus (Department of Natural Sciences, Bishop Museum, Honolulu, USA) and Dávid Rédei (Nankai University, Tianjin, China) kindly provided their critical comments on the manuscript. The work was financially supported by project of the Ministry of Culture of the Czech Republic (DKRVO 2019-2023/5.I.b, MK000023272) to the National Museum, Prague.

\section{References}

AGASSIZ L. 1847: Nomenclatoris Zoologici index universalis, continens nomina systematica classium, ordinum, familiarum et generum animalium omnium tam viventium quam fossilium, secundum ordinem alphabeticum unicum disposita, adjectis synonymiis plantarum, nec non variis adnotationibus et emendationibus. Jent et Gassmann, Soloduri, [1846], viii + 393 pp.

AGUESSE P., DAKKI M., GHEIT A. \& RAMDANI M. 1982: Les Heteropteres aquatiques du Maroc. Inventaire commente. Bulletin de l'Institut Scientifique (Rabat) 6: 125-138.

AKRAMOVSKAYA E. G. 1959: Nastoyashchie poluzhestkokrylye (Hemiptera-Heteroptera) Armyanskoy SSR. [True bugs (Hemiptera-Heteroptera) of Armenian SSR]. Materialy po Izucheniyu Fauny Armyanskoy SSR 4: 79-144.

ALAYO P. D. 1971: Los Hemipteros de Cuba. Adiciones y enmiendas. Trabajos de Divulgacion, Museo "Felipe Poey" de la Academia de Ciencias de Cuba 63: 1-17.

ALAYO P. D. 1974: Los Hemipteros acuaticos de Cuba. Torreia, Nueva Serie 36: 1-64 + 1 table.

AMYOT C. J. B. \& SERVILLE J. G. A. 1843: Histoire naturelle des insectes. Hémiptères. Roret, Paris, lxxiv $+675+6$ pp.

ANDERSEN N. M. 1995: Phylogeny and classification of aquatic bugs (Heteroptera, Nepomorpha). An essay review of Mahner's ,Systema Cryptoceratum Phylogeneticum'. Entomologica Scandinavica 26: 159-166.

ANDERSEN N. M. \& WEIR T. A. 2004: Australian water bugs. Their biology and identification (Hemiptera-Heteroptera, Gerromorpha \& Nepomorpha). Entomograph Vol. 14. Apollo Books, Stenstrup \& CSIRO Publishing, Collingwood, 344 pp.

ANONYMUS 2019: Coreoidea Species File Online. Version 5.0/5.0. (Last access: December 10, 2019). (http://Coreoidea.SpeciesFile.org).

AUKEMA B., RIEGER CH. \& RABITSCH W. 2013: Catalogue of the Heteroptera of the Palaearctic Region. VI. Supplement. The Netherlands Entomological Society, Amsterdam, xxiii + 629 pp.

BACHMANN A. O. 1998: Heteroptera acuáticos. Pp. 163-180. In: MORRONE J. J. \& COSCARÓN S. (eds): Biodiversidad de Artrópodos Argentinos. Una Perspectiva Biotaxonómica. Ediciones Sur, La Plata, vii +559 pp.

BACHMANN A. O. 1999: Catálogo de los tipos de Heteroptera (Insecta) conservados en el Museo Argentino de Ciencias Naturales. Revista del Museo Argentino de Ciencias Naturales, Nueva Serie 1: 191-230.

BACHMANN A. O. \& MAZZUCCONI S. A. 1995: Insecta Heteroptera (= Hemiptera s. str.). Pp. 1291-1325. In: LOPRETTO E. C. \& TELL G. (eds): Ecosistemas de aguas continentales. Metodologías para su estudio. Vol. 3. Ediciones Sur, La Plata, pp. xvii + 897-1401.

BACHMANN A. O. \& MAZZUCCONI S. A. 2017: Family Ochteridae Kirkaldy. Pp. 203-204. In: COSCARÓN M. DEL C. (ed.): A catalogue of the Heteroptera (Hemiptera) or true bugs of Argentina. Zootaxa 4295(1): 1-432.

BAEHR M. 1989: Review of the Australian Ochteridae (Insecta, Heteroptera). Spixiana 11: 111-126.

BAEHR M. 1990a: Revision of the genus Megochterus Jaczewski (Insecta: Heteroptera: Ochteridae). Invertebrate Taxonomy 4: 197-203

BAEHR M. 1990b: Revision of the genus Ochterus Latreille in the Australian Region (Heteroptera: Ochteridae). Entomologica Scandinavica 20: 449-477.

BAEHR M. 1990c: Ochterus noualhieri sp. n., a new ochterid from Java [Heteroptera, Ochteridae]. Revue Française d'Entomologie (Nouvelle Série) 12: 91-93.

BAENA M. \& VÁZQUEZ M. A. 1986: Catálogo preliminar de los Heterópteros acuáticos ibéricos. Graellsia 42: 61-89.

BAENA RUIZ M. \& FERRERAS ROMERO M. 1982: Heterópteros acuáticos (Het. Nepomorpha, Gerromorpha) de la Sierra de los Santos y Sierra de Córdoba. Boletín de la Asociación Española de Entomología 6: 137-145.

BAL A. \& BASU R. C. 1998: Insecta: Hemiptera: Water-bugs. Pp. 431-461. In: Fauna of Meghalaya, Part 4. State Fauna Series. Vol. 4. Zoological Survey of India, Kolkata, iv $+485 \mathrm{pp}$.

BAL A. \& BASU R. C. 2000: Insecta: Hemiptera: Water-bugs. Pp. 427-443. In: Fauna of Tripura, Part 2. State Fauna Series. Vol. 7. 
Zoological Survey of India, Kolkata, iv +443 pp.

BAL A. \& BASU R. C. 2004: Insecta: Hemiptera: Water-bugs. Pp. 293-310. In: Fauna of Manipur, Part-2 (Insects). State Fauna Series. Vol. 10. Zoological Survey of India, Kolkata, v +625 pp.

BAL A. \& BASU R. C. 2007: Insecta: Hemiptera: Water-bugs. Pp. 249-260. Fauna of Mizoram. State Fauna Series. Vol. 14. Zoological Survey of India, Kolkata, 691 pp.

BARBER H. G. 1913. Description of two new species of Ochterus Latr. (Hemiptera) with an arrangement of the North American species. Canadian Entomologist 45: 213-215.

BARBER H. G. 1914: Insects of Florida. II. Hemiptera. Bulletin of the American Museum of Natural History 33: 495-535.

BARBOSA J. F. \& RODRIGUES H. D. D. 2015: The true water bugs (Nepomorpha). Pp. 159-199. In: PANIZZI A. R. \& GRAZIA J. (eds): True bugs (Heteroptera) of the Neotropics. Springer Science + Business Media, Dordrecht, xxii + 901 pp.

BASS D. 2003: A survey of freshwater macroinvertebrates in Tobago. Living World, Journal of the Trinidad and Tobago Field Naturalist's Club 2003: 64-69

BATELKA J. 2012: Socotra Archipelago - a lifeboat in the sea of changes: advancement in Socotran insect biodiversity survey. Pp. 1-26. In: HÁJEK J. \& BEZDĚK J. (eds): Insect biodiversity of the Socotra Archipelago. Acta Entomologica Musei Nationalis Pragae 52 (Supplementum 2): i-vi + 1-557.

BENNETT D. V. \& COOK E. F. 1981: The semiaquatic Hemiptera of Minnesota (Hemiptera: Heteroptera). Agricultural Experiment Station University of Minnesota, Technical Bulletin 332: 1-59.

BERGROTH E. E. 1890a: 'Communications.M. le Secrétaire donne lecture de deux notes hémiptérologiques adressées de Forssa (Finlande) par M. le D ${ }^{\mathrm{r}}$ E. Bergroth'. Bulletin de la Société Entomologique de France 1890: 1xv-1xvi.

BERGROTH E. E. 1890a: Rectifications. Bulletin de la Société Entomologique de France 1890: cxix.

BERGROTH E. 1914: H. Sauter's Formosa-Ausbeute; Hemiptera Heteroptera I. Aradidae, Pyrrhocoridae, Myodochidae, Tingidae, Reduviidae, Ochteridae. Entomologische Mitteilungen 3: 353-364.

BERTRAND H. 1962: Mission H. Bertrand (1956-1957) en Afrique occidentale et équatoriale. Hydrocorises. Bulletin de l'Institut Français d'Afrique Noire, Série A 34: 880-885.

BEUTEL R. G. \& LESCHEN R. A. B. (eds) 2016: Handbook of zoology. Vol. 4. Arthropoda: Insecta. Coleoptera, Beetles. Morphology and systematics. Archostemata, Adephaga, Myxophaga, and Polyphaga partim. Vol. 1. 2nd Edition. Walter de Gruyter, Berlin, Boston, xvii $+684 \mathrm{pp}$.

BEZDĚK J. \& HÁJEK J. 2017: Insect biodiversity of the Socotra Archipelago - underlined and counted. Pp. 1-39. In: HÁJEK J. \& BEZDĚK J. (eds): Insect biodiversity of the Socotra Archipelago III. Acta Entomologica Musei Nationalis Pragae 57 (supplementum): i-vi+1-225.

BEZDĚK J., PURCHART L., KRÁL K. \& HULA V. 2012: List of local Socotran geographical names used in entomological literature. Pp. 27-67. In: HÁJEK J. \& BEZDĚK J. (eds): Insect biodiversity of the Socotra Archipelago. Acta Entomologica Musei Nationalis Pragae 52 (Supplementum 2): i-vi + 1-557.

BIONDI M., IANNELLA M. \& D’ALESSANDRO P. 2019: Unravelling the taxonomic assessment of an interesting new species from Socotra Island: Blepharidina socotrana sp. nov. (Coleoptera: Chrysomelidae). Acta Entomologica Musei Nationalis Pragae 59: 499-505.

BLANCHARD E. 1840: Histoire naturelle des insectes. Vol. III. Orthoptères, Neuroptères, Hémiptères, Hymenoptères, Lépidoptères et Diptères. Dumeril, Paris, 672 pp.

BLATCHLEY W. S. 1926: Heteroptera or true bugs of Eastern North America with especial reference to the faunas of Indiana and Florida. Nature Publishing Company, Indianapolis, 1116 pp.

BOBB M. L. 1951: Life history of Ochterus banksi Barber (Hemiptera: Ochteridae). Bulletin of the Brooklyn Entomological Society 46 92-100.

BOBB M. L. 1974: The aquatic and semi-aquatic Hemiptera of Virginia. The Insects of Virginia. No. 7. Virginia Polytechnic Institute and State University, Research Division Bulletin 87: i-iv + 1-195.

BOLÍVAR I. 1879: Hemípteros nuevos del Museo de Madrid. Anales de la Sociedad Española de Historia Natural 8: 130-146.
BOULARD M. \& COFFIN J. 1991: Sur la biologie juvénile d'Ochterus marginatus (Latreille, 1804) camouflage et construction (Hemiptera: Ochteridae). EPHE, Travaux Laboratoire Biologique d'Evolution des Insectes 4: 57-68.

BROWN E. S. 1951: Aquatic and semiaquatic insects. Pp. 221-273. In: Expedition to S. W. Arabia, 1937-1938. Vol. 1. British Museum (Natural History), London.

BROŻEK J. 2013: A comparison of external and internal maxilla and mandible morphology of water bugs (Hemiptera: Heteroptera: Nepomorpha). Zootaxa 3635(4): 340-378.

BROŻEK J. 2015: Phylogenetic signals from Nepomorpha (Insecta: Hemiptera: Heteroptera) mouthparts: stylets bundle, sense organs, and labial segments. Scientific World Journal 2014(237854): 1-30.

BRULLÉ A. 1836: Histoire naturelle des insectes, traitant de leur organisation et de leurs moeurs en général, et comprenant leur classification et la description des espèces. Orthoptères et Hémiptères. Vol. IX (2 parts). Pillot, Paris, [1835], 415 pp.

BURMEISTER H. C. C. 1835: Handbuch der Entomologie. Zweiter Band. Besondere Entomologie. Erste Abtheilung. Schnabelkerfe, Rhynchota. Enslin, Berlin, xii +400 pp.

CAI W.-ZH., CUI J.-X., LIU G.-Q. \& WANG J.-SH. et al. 2016: Insect fauna of Henan. Hemiptera: Heteroptera. Science Press, Beijing, XXviii +783 pp. + pls. XXVIII.

CAMPOS R. F. 1925: Nueva contribucion al estudio de las Rincotos Heteropteros del Ecuador. Revista del Colegio Nacional Vicente Rocafuerte 7 (19-20): 43-67.

CARAPEZZA A. 1997: Heteroptera of Tunisia. Naturalista Siciliano, Serie Quarta 21 (Suppl. A): 1-331 pp. + pls. I-XVI.

CARLINIA. DE 1895: VII. Rincoti. In: Esplorazione del Giuba e dei suoi affluenti compiuta dal Cap. V. Bottego durante gli anni 1892-93 sotto gli auspici della Societa Geografica Italiana. Annali del Museo Civico di Storia Naturale di Genova, Serie 2 15: 103-125.

CASSIS G. \& GROSS G. F. 1995: Hemiptera: Heteroptera (Coleorrhyncha to Cimicomorpha). In: HOUSTON W. W. K. \& MAYNARD G. V. (eds): Zoological Catalogue of Australia. Vol. 27.3A. Melbourne, CSIRO Publishing, $\mathrm{xv}+506 \mathrm{pp}$.

ÇERÇİ B., ÖZGEN İ. \& DIOLI P. 2018: Additional faunistic notes on Heteroptera (Hemiptera: Insecta) in East Anatolia (Turkey). Journal of Entomology and Zoology Studies 6: 1225-1231.

CHAMPION G. C. 1897-1901: Insects. Rhynchota. Hemiptera - Heteroptera. Vol. II. In: GODMAN F. D. \& SALVIN O. (eds): Biologia Centrali-Americana. Vol. II. R. H. Porter, London, xvi + 416 pp., 22 pl. [1901: i-xvi, 345-416]

CHANDRA K. \& JEHAMALAR E. E. 2012: Ochterus nicobarensis sp. nov. from Great Nicobar Biosphere Reserve, Andaman and Nicobar Islands, India (Hemiptera: Heteroptera: Ochteridae). Acta Entomologica Musei Nationalis Pragae 52: 23-28.

CHAPMAN H. C. 1958: Notes on the identity, habitat and distribution of some semi-aquatic Hemiptera of Florida. Florida Entomologist 41: $117-124$.

CHAPMAN H. C. 1959: Distributional and ecological records for some aquatic and semi-aquatic Heteroptera of New Jersey. Bulletin of the Brooklyn Entomological Society 54: 8-12.

CHEN P.-P. 1999: Hemiptera: Ochteridae. Pp. 9-10. In: HUANG B. K. (ed.): Fauna of insects, Fujian Province of China. Vol. 2. Fujian Science and Technology Press, Fuzhou, 806 pp.

CHEN P.-P., NIESER N. \& ZETTEL H. 2005: The aquatic and semi-aquatic bugs (Heteroptera: Nepomorpha \& Gerromorpha) of Malesia. Fauna Malesiana Handbook, Vol. 5. Brill, Leiden - Boston, $\mathrm{x}+546 \mathrm{pp}$.

CHEN Z.-Y., LIANG G.-Q., JIA F.-I., ZIE W.-C., CHEN Z.-M., DENG S.-F., TANG C.-G., CHEN J.-I. \& YANG Q.-Y. 2001: A catalog of insects from Dadongshan (Mt.) of Guangdong Nanlin National Nature Reserve. Ecological Science 20(4): 42-47 (in Chinese, English abstract).

CHENG L., YANG CH. M., LI D.-Q. \& LIU H.-M. 2006: Aquatic Heteroptera (Insecta: Gerromorpha and Nepomorpha) from Xishuangbanna, Yunnan, China. Raffles Bulletin of Zoology 54: 203-214.

CHINA W. E. \& MILLER N. C. E. 1950: The 3rd Danish expedition to Central Asia. Zoological results 3. Hemiptera (Insecta) from Afghanistan. Videnskabelige Meddelelser fra Dansk Naturhistorisk Forening 112: 225-234. 
CHINA W. E. \& MILLER N. C. E. 1955: Check-list of family and subfamily names in Hemiptera-Heteroptera. Annals and Magazine of Natural History, Series 12 8: 257-267.

CHINA W. E. \& MILLER N. C. E. 1959: Check-list and keys to the families and subfamilies of the Hemiptera-Heteroptera. Bulletin of the British Museum (Natural History), Entomology 8(1): 1-45.

CIANFERONI F. 2012. The Ochteridae of Ecuador, with new records and an identification key (Hemiptera: Heteroptera: Nepomorpha). Zootaxa 3260: 62-68.

CIANFERONI F. \& TERZANI F. 2013: Nuovi dati su Gerromorpha e Nepomorpha in Italia (Hemiptera Heteroptera). Bollettino della Società Entomologica Italiana 145: 51-57.

COBBEN R. H. 1960: The Heteroptera of the Netherlands Antilles. III. Saldidae. Studies on the Fauna of Curaçao and Other Caribbean Islands 11 (52): 44-61.

CORDEIRO I. DA R. S., MOREIRA F. F. F. \& SILVA F. A. C. DA 2014: A new Ochterus (Hemiptera: Heteroptera: Ochteridae) from northeastern Brazil, with a key to the species recorded from the country. Zootaxa 3860(5): 493-497.

COSTAA. 1862: Additamenta ad Centurias Cimicum regni Neapolitani. $41 \mathrm{pp}$. [Also published in: Atti del Reale Istituto d'Incorragiamento alle Scienze Naturali Napoli 10 [1863]: 329-367 + pls I-III.]

DAMGAARD J. \& ZETTEL H. 2014: The water bugs (Hemiptera-Heteroptera: Gerromorpha \& Nepomorpha) of New Caledonia: Diversity, ecology and biogeographical significance. Pp. 219-238. In: GUILBERT É., ROBILLARD T., JOURDAN H. \& GRAND COLAS P. (eds): Zoologia Neocaledonica 8. Biodiversity studies in New Caledonia. Muséum national d'Histoire naturelle, Paris, 315 pp.

DISTANT W. L. 1906: Rhynchota. Vol III. (Heteroptera-Homoptera). In: BINGHAM C. T. (ed.): The fauna of British India including Ceylon and Burma. Taylor and Francis, London, xiv +503 pp.

DISTANT W. L. 1910: Rhynchota. Vol V. Heteroptera: Appendix. In: SHIPLEY A. E. \& MARSHALL G. A. K. (eds): The fauna of British India including Ceylon and Burma. Taylor and Francis, London, xii +362 pp.

DISTANT W. L. 1915: A few undescribed Rhynchota. Annals and Magazine of Natural History, Series 8 15: 503-507.

DISTANT W. L. 1920: Rhynchota from New Caledonia. Annals and Magazine of Natural History, Series 9 6: 143-194.

DITRICH T., PETR J., LEPŠ J. Š. \& KMENT P. 2020: In memoriam of Professor Miroslav Papáček (1953-2019): biography, memories, bibliography and list of described taxa. Acta Entomologica Musei Nationalis Pragae 60: 1-14.

DOHRN A. 1859: Catalogus Hemipterorum. Herrcke \& Lebeling, Stettin, vii $+112 \mathrm{pp}$.

DRAKE C. J. 1952: Concerning American Ochteridae (Hemiptera). Florida Entomologist 35: 72-75.

DRAKE C. J. \& GÓMEZ-MENOR J. 1954: A new genus of American Ochteridae (Hemiptera). EOS, Revista Española de Entomologia 30: $157-159+$ pl. X.

DUFOUR L. 1833: Recherches anatomiques et physiologiques sur les Hémiptères, accompagnées de considerations relatives à l'histoire naturelle et à la classification de ces insectes. Mémoires Présentés par Diverses Savants à l'Académie des Sciences de l'Institut de France 4: $129-462+$ pls. I-XIX.

DURSUN A. \& FENT M. 2018: Contributions to Nepomorpha (Insecta: Hemiptera: Heteroptera) fauna in wetland areas of Amasya, Turkey. Acta Biologica Turcica 31: 193-202.

ECKERLEIN H. \& WAGNER E. 1965: Ein Beitrag zur Heteropterenfauna Algeriens. Acta Faunistica Entomologica Musei Nationalis Pragae 11: 195-243.

ELDER J.-F. 2017: Les Hétéroptères aquatiques et semi-aquatiques de France: analyse bibliographique commentée des connaissances actuelles sur la distribution des espèces par département. (Heteroptera, Nepomorpha, Gerromorpha). Ephemera 18(2): 69-133.

EPLER J. H. 2006: Identification manual for the aquatic and semi-aquatic Heteroptera of Florida (Belostomatidae, Corixidae, Gelastocoridae, Gerridae, Hebridae, Hydrometridae, Mesoveliidae, Naucoridae, Nepidae, Notonectidae, Ochteridae, Pleidae, Saldidae, Veliidae) Florida Department of Environmental Protection, Tallahassee, 194 pp.

ESAKI T. 1926: Verzeichniss der Hemiptera-Heteroptera der Insel Formosa. Annales Musei Nationalis Hungarici 24: 136-189.
EVANS J. W. 1971: Some Upper Triassic Hemiptera from Mount Crosby, Queensland. Memoirs of the Queensland Museum 16: 145-151.

FAÚNDEZ E. I. \& ASHWORTH A. C. 2015: Notas sobre la familia Gelastocoridae (Hemiptera: Heteroptera) en el extremo sur de Chile, con descripción de un subgénero y especie nuevos. Anales Instituto Patagonia 43: 69-74

FAÚNDEZ E. I. \& CARVAJAL M. A. 2017: New data on the subgenus Rhinodermacoris (Insecta: Heteroptera: Gelastocoridae), with comments on its Gondwanian origin and relationships. Pp. 321-324. In: GUAIQUIL I., LEPPE M., ROJAS P. \& CANALES R. (eds): Visiones sobre Ciencia Antárctica. Libro de Resúmenes del IX Congreso Latinoamericano de Ciencia Antárctica. Punta Arenas, Chile, 4 al 6 de octubre 2017. Instituo Antártico Chileno, Punta Arenas, 931 pp.

FENT M. \& DURSUN A. 2018: Erster präzise lokalisierter Nachweis für Ochterus marginatus marginatus (Latreille, 1804) im Gebiet von Thrakien (Türkei). Heteropteron 52: 22-24.

FENT M., KMENT P., ÇAMUR-ELIPEK B. \& KIRGIZ T. 2011: Annotated catalogue of Enicocephalomorpha, Dipsocoromorpha, Nepomorpha, Gerromorpha, and Leptopodomorpha (Hemiptera: Heteroptera) of Turkey, with new records. Zootaxa 2856: 1-84.

FIEBER F. X. 1851: Genera Hydrocoridarum secundum ordinem naturalem in familias disposita. Calve, Pragae, $31 \mathrm{pp}$. [Preprint of Abhandlungen der Böhmischen Gesellschaft der Wissenschaften 7 [1852]: 178-211.]

FIEBER F. X. 1861: Die europäischen Hemiptera. Halbflügler (Rhynchota Heteroptera). Carl Gerold's Sohn, Wien, pp. 113-444.

FROESCHNER R. C. 1981: Heteroptera or true bugs of Ecuador: A partial catalog. Smithsonian Contributions to Zoology 322: 1-147.

FROESCHNER R. C. 1999: True bugs (Heteroptera) of Panama: A synoptic catalog as a contribution to the study of Panamanian biodiversity. Memoirs of the American Entomological Institute 61: i-ii + 1-393.

GAPUD V. P. 1981: Contribution to the taxonomy of the genus Ochterus Latreille (Hemiptera: Ochteridae). Kalikasan, Philippine Journal of Biology 10(2-3): 300-309.

GAPUD V. P. 1986: Philippine water bugs. Guide to Philippine Flora and Fauna 8: 1-47.

GAPUD V. P. 1995: A new species of Ochterus Latreille (Hemiptera: Ochteridae) from the Philippines. Asia Life Sciences 4(1): 41-44.

GAPUD V. P. 2003: Two new Philippine Ochterus Latreille (Insecta: Heteroptera: Ochteridae) and checklist of Philippine species. Annalen des Naturhistorischen Museums in Wien, Serie B 104 [2002]: 99-108.

GAPUD V. P. \& SAN VALENTIN H. O. 1977: The Ochteridae (Hemiptera) of the Philippines. Kalikasan, Philippine Journal of Biology 6(3): 269-300.

GARCÍA ROJAS A. M., FERRERAS ROMERO M. \& MORILLO ORTIZ R. 1987: Nuevos datos sobre Coleópteros Hidrocántaros (Adephaga Aquatica) y Heterópteros acuáticos (Het.: Nepomorpha, Gerromorpha) de Sierra Morena: río Yeguas. Boletín de la Asociación Española de Entomología 11: 207-213.

GEORGHIOU G. P. 1977: The insects and mites of Cyprus. Benaki Phytopathological Institut, Kiphissia, Athens, Greece, 347 pp.

GHAHARI H., MOULET P., OSTOVAN H. \& LINNAVUORI R. E. 2013: An annotated catalog of the Iranian Dipsocoromorpha, Enicocephalomorpha, Gerromorpha, Leptopodomorpha and Nepomorpha (Hemiptera: Heteroptera). Zootaxa 3641(4): 301-342.

GHEIT A. 1995: Catalogue des Hémipteres Hydrocorises et Amphibiocorses des provinces Nord Marocaines (1 re note). Entomologiste (Paris) 51: 241-249.

GHOSH L. K., BISWAS B., CHAKRABORTY S. P. \& SEN G. C. 1989 : Insecta: Hemiptera. Pp. 181-224. In: JAIRAPURI M. SH. (ed.): Fauna of Orissa. Part 2. State Fauna Series. Vol. 1. Zoological Survey of India, Kolkata, 318 pp.

GÖLLNER-SCHEIDING U. \& ARNOLD K. 1988: Sammelausbeute von Heteropteren aus dem südwestlichen Bulgarien (Insecta). Faunistische Abhandlungen Staatliches Museum für Tierkunde Dresden 15: $137-154$.

GRIMALDI D. A. \& ENGEL M. S. 2005: Evolution of the Insects. Cambridge University Press, Cambridge, New York, Melbourne, Madrid, Cape Town, Singapore, São Paulo, xv +715 pp.

GUÉRIN-MÉNEVILLE F. E. 1843: Note sur la Naucoris rugosa de J. Desjardins, formant un nouveau genre d'Hémiptères, et description 
de plusieurs espèces des genres Pelogonus et Mononyx. Revue Zoologique Société Cuvier 6: 112-114.

GÜNTHER CH. \& GÜNTHER H. 2019: List of bugs collected in Southern Spain (Heteroptera). Entomologische Zeitschrift (Schwanfeld) 129: $223-242$.

HÁJEK J. \& BEZDĚK J. (eds) 2012: Insect biodiversity of the Socotra Archipelago. Acta Entomologica Musei Nationalis Pragae 52 (Supplementum 2): i-vi + 1-557.

HÁJEK J. \& BEZDĚK J. (eds) 2014: Insect biodiversity of the Socotra Archipelago II. Acta Entomologica Musei Nationalis Pragae 54 (Supplementum): i-vi + 1-440.

HÁJEK J. \& BEZDĚK J. (eds) 2017: Insect biodiversity of the Socotra Archipelago III. Acta Entomologica Musei Nationalis Pragae 57 (Supplementum): i-vi + 1-225.

HÁJEK J. \& BEZDĚK J. 2019: Annotated catalogue of beetles (Coleoptera) of the Socotra Archipelago. Zootaxa 4715(1): 1-76.

HALE H. M. 1925: Results of Dr. E. Mjöberg's Swedish Scientific Expeditions to Australia 1910-1913. 44. The aquatic and semi-aquatic Hemiptera. Arkiv för Zoologi 17A (20): 1-19.

HAMID A. \& HAMID B. L. 1973: A new record of Ochterus marginatus (Latr.) (Heteroptera: Ochteridae) from Sind province, Pakistan. Pakistan Journal of the Scientific and Industrial Research 16: 48-49.

HAYASHI M. 1995: Occurrence of Ochterus flavomarginatus (Heteroptera, Ochteridae) in Hokkaido. Rostria 44: 20 (in Japanese, English title).

HAYASHI M. \& MIYAMOTO S. 2018: Hemiptera. Pp. 329-427. In: KAWAI T. \& TANIDA K. (eds): Aquatic Insects of Japan: Manual with Keys and illustrations. The second edition. Tokai University Press, Kanagawa, 1752 pp (in Japanese).

HAYASHI M., TOMOKUNI M., YOSHIZAWA K. \& ISHIKAWA T. (eds) 2016: Catalogue of the insects of Japan. Vol. 4, Paraneoptera. Entomological Society of Japan \& Touka-shobo, Fukuoka, 629 pp.

HEBSGAARD M. B., ANDERSEN N. M. \& DAMGAARD J. 2004: Phylogeny of the true water bugs (Nepomorpha: Hemiptera-Heteroptera) based on $16 \mathrm{~S}$ and 28S rDNA and morphology. Systematic Entomology 29: 488-508.

HECKMAN 2011: Encyclopedia of South American aquatic insects: Hemiptera - Heteroptera. Illustrated keys to known families, genera, and species in South America. Springer, Dordrecht, Heidelberg, London, New York, ix +679 pp.

HERRERA MADRIGAL F. 2015: First record of the genus Ocyocherus from Colombia and new records of Ochteridae from Costa Rica (Hemiptera: Heteroptera). Acta Biológica Colombiana 20: 255-257.

HERRICH-SCHÄFFER G. A. W. 1849-1853: Die Wanzenartigen Insecten. Neunter und letzter Band. J. L. Lotzbeck, Nürnberg, 348 pp + pls. CCLXXXIX-CCXXIV. [1849: pp. 1-44]

HESSE A. J. 1925: Contributions to the knowledge of the fauna of South-West Africa. IV. A list of the Heteropterous and Homopterous Hemiptera of South-West Africa. Annals of the South African Museum 23(1): 1-190.

HIURA I. 1967: Biogeographical studies on the aquatic and semiaquatic Heteroptera of Japan, 1. A list of specimens in the collection of the Osaka Museum of Natural History, with some notes on their geographical distribution. Bulletin of the Osaka Museum of Natural History 20: 65-81.

HOBERLANDT L. 1952: Results of the zoological scientific expedition of the National Museum in Praha to Turkey. 2. Hemiptera-Heteroptera I. The aquatic and semiaquatic Heteroptera of Turkey. Acta Entomologica Musei Nationalis Pragae 26(352) [1948-1950]: 1-74 + pls. I-XVII.

HOBERLANDT L. 1974: Results of the Czechoslovak-Iranian entomological expedition to Iran 1970. No. 1: Introduction. Acta Entomologica Musei Nationalis Pragae, Supplementum 6: 9-20.

HOBERLANDT L. 1981: Results of the Czechoslovak-Iranian entomological expedition to Iran. Introduction to the Second expedition. Acta Entomologica Musei Nationalis Pragae 40: 5-32.

HOBERLANDT L. 1983: Results of the Czechoslovak-Iranian entomological expedition to Iran. Introduction to the Third expedition 1977 Acta Entomologica Musei Nationalis Pragae 41: 5-24.

HOFFMAN R. L. 2012: Velvety Shore Bugs in Virginia (Heteroptera: Ochteridae). Banisteria 40: 58-60.

HOFFMANN W. E. 1933: A preliminary list of the aquatic and semi-aqua- tic Hemiptera of China, Chosen (Korea), and Indo-China. Lingnan Science Journal 12 (Supplement): 243-258.

HOFFMANN W. E. 1941: Catalogue of aquatic Hemiptera of China, Indo-China, Formosa, and Korea. Lingnan Science Journal 20: 1-78 $+78 \mathrm{~A}-78 \mathrm{E}$

HORVÁTH G. 1899: Hemiptera Caucasica. Pp. 456-472. In: RADDE G. (ed.): Die Sammlungen des Kaukasischen Museums. Band I. Zoologie. Typographie der Kanzelie des Landeschefs, Tiflis, xvi + 520 pp.

HORVÁTH G. 1913: Aquatic and semi-aquatic Rhynchota from the Lake of Tiberias and its immediate vicinity. Journal of the Asiatic Society of Bengal 9: 477-480.

HUA L.-ZH. 2000: XVIII. Order Hemiptera, Pp. 162-216. In: HUA L.-Zн.: List of Chinese Insects. Vol. I. Guangdong, P. R. of China, 448 pp.

HUNGERFORD H. B. 1919: The biology and ecology of aquatic and semiaquatic Hemiptera. Kansas University Science Bulletin 11: i-iii (colour pls) + 3-328 + 337-341 (index).

HUNGERFORD H. B. 1927: A report upon the aquatic and semi-aquatic Hemiptera of the Mulford Biological Expedition to Bolivia, South-America, 1921-22. Proceedings of the Entomomological Society of America 29: 187-190+ pl. 10 .

HUNGERFORD H. B. \& BEAMER R. H. 1925: Report of collections of aquatic Hemiptera taken in Cherokee County, Kansas, and other new records from the state. Entomological News 36: 262-266, 295-299.

ICZN 1985: International Code of Zoological Nomenclature. Third edition. International Trust for Zoological Nomenclature, London, $388 \mathrm{pp}$.

ICZN 1999: International Code of Zoological Nomenclature. Fourth edition. International Trust for Zoological Nomenclature, London, $306 \mathrm{pp}$.

JACZEWSKI T. 1934: Notes on the Old World species of Ochteridæ (Heteroptera). Annals and Magazine of Natural History, Series 10 13: 597-613.

JACZEWSKI T. 1935: Die Uferwanzen (Heteroptera: Fam. Leptopodidae, Acanthiidae, Ochteridae und Mononychidae) der Deutschen Limnologischen Sunda-Expedition. Archiv für Hydrobiologie, Suppl.-Band XIII 'Tropische Binnengewässer' 5: 474-483.

JACZEWSKI T. 1938: Eine neue Ochteriden-Art aus Africa, nebst faunistischen Angaben ueber einige andere Arten dieser Familie (Heteroptera). Sitzungsberichte der Gesellschaft der Naturforschender Freunde in Berlin 1937: 186-187.

JACZEWSKI T. 1949: Notes on Corixidae. XXIV-XXVIII. Polskie Pismo Entomologiczne 19: 103-113.

JELL P. A. 2004: The fossil insects of Australia. Memoirs of the Queensland Museum 50: 1-124.

JOHNSON C. W. 1930: A list of the insect fauna of Nantucket, Massachusetts. Publications of the Nantucket Maria Mitchell Association 3(2): $1-38$.

JOSIFOV M. 1954: Novi i redki za b'lgarskata fauna khemipteri Hemiptera-Heteroptera. (Neue un seltene für die Bulgarische Fauna Hemiptera - Heteroptera). Izvestiya na Zoologicheskiya Institut (Sofiya) 3: 237-244 (in Bulgarian, Russian and German summaries).

JOSIFOV M. 1959: Hemiptera - Heteroptera ot ostrova Tasos. (Hemiptera-Heteroptera von der Insel Thasos). Izvestiya na Zoologicheskiya Institut (Sofiya) 8: 265-269 (in Bulgarian, Russian and German summaries).

JOSIFOV M. 1960: Vidov s'stav i razprostranenie na nasekomite ot razreda Heteroptera v B'lgariya, chast I. (Artenzusammensetzung und Verbreitung der Insekten von der Ordnung Heteroptera in Bulgarien). Izvestiya na Zoologicheskiya Institut (Sofiya) 9: 107-177 (in Bulgarian, Russian and German summaries).

JOSIFOV M. 1963: Polukrili nasekomi (Heteroptera) ot okolnostite na Petrich (yugozapadna B'lgariya). (Heteropteren aus der Umgebung von Petrič (SW Bulgarien)). Izvestiya na Zoologicheskiya Institut s Muzey (Sofiya) 13: 93-132 (in Bulgarian, Russian and German summaries).

JOSIFOV M: 1986: Verzeichnis der von der Balkanhalbinsel bekannten Heteropterenarten (Insecta, Heteroptera). Faunistische Abhandlungen Staatliches Museum für Tierkunde Dresden 14: 61-93.

JOSIFOV M. \& KERZHNER I. M. 1972: Heteroptera aus Korea. I. Teil (Ochteridae, Gerridae, Saldidae, Nabidae, Anthocoridae, Miridae, Tingidae und Reduviidae). Annales Zoologici (Warszawa) 29: 148-180.

KANYUKOVA E. V. 1997: Otryad poluzhestkokrylye ili klopy (Heteroptera). [Order true bugs (Heteroptera)]. Pp. 265-288, 400-423. In: 
TSALOLIKHIN S. J. (ed.): Opredelitel' presnovodnykh bespozvonochnykh Rossii i sopredel'nykh territoriy. Tom. 3. Paukoobraznye. Nizshie nasekomye. [Key to freshwater invertebrates of Russia and adjacent lands. Vol. 3. Chelicerata. Lower insects]. St. Petersburg, 444 pp (in Russian, English title).

KANYUKOVA E. V. 2006: Vodnye poluzhestkokrylye nasekomye (Heteroptera: Nepomorpha, Gerromorpha) fauny Rossii i sopredel'nykh stran. (Aquatic and semiaquatic bugs (Heteroptera: Nepomorpha, Gerromorpha) of the fauna of Russia and neighbouring countries). Dalnauka, Vladivostok, 296 pp (in Russian, English abstract).

KANYUKOVA E. V. \& MARUSIK YU. M. 2006: A checklist of Heteroptera of the Kuril Islands and brief zoogeographical survey of the fauna. Biodiversity and Biogeography of the Kuril Islands and Sakhalin 2: 159-172.

KATBEH A., CARAPEZZA A. \& AKKAWI M. 2000: Heteroptera of Jordan: Specimens preserved in the University of Jordan Insects Museum (Insecta). Atti dell'Accademia Roveretana degli Agiati, Seria VII 10B: 111-137.

KATIYAR K. N. 1953: Part 19. - Order Hemiptera (continued). Family Pelogonidæ, Naucoridæ, Belostomatidæ and Nepidæ. Pp. 135-136. In: ROONWAL M. L., MATHUR R. N., BHASIN G. D., SINGH B., PANT G. D., CHANDRA A., KATIYAR K. N. \& MATHUR R. K (eds): A systematic catalogue of the main identified entomological collectioncollection at the Forest Research Institute, Dehra Dun. Indian Forest Leaflet 121(3): 39-187.

KERZHNER I. M. 1978: Poluzhestkokrylye (Heteroptera) Sakhalina i Kuril'skikh ostrovov. [True bugs (Heteroptera) of the Sakhalin and the Kuril Islands.] In: Novye dannye o nasekomykh Sakhalina i Kuril'skikh ostrovov. [New data on insects of Sakhalin and Kurile Islands.] Trudy Biologo-Pochvennogo Instituta Dal'nevostochnogo Otdeleniya Akademii Nauk SSSR, Novaya Seriya 50: 31-57 (in Russian).

KERZHNER I. M., KANYUKOVA E. V., MARUSIK YU. M., URBAIN B. K., NAKAMURA M. \& LELEJ A. S. 2004: Heteroptera of the Kuril Islands: material collected by the International Expedition 1994-1999 and updated checklist. Zoosystematica Rossica 12: 231-242.

KERZHNER I. M. \& MARUSIK YU. M. 1997: Heteroptera (Insecta) of the Kurile Islands: a revised check-list and new data on Middle Kuriles. Russian Entomological Journal 5: 21-29.

KIRITSHENKO A. N. 1918: Poluzhestkokrylyya (Hemiptera-Heteroptera) Kavkazskago Kraya. Chast' I. (Hemiptera-Heteroptera faunae caucasiae. Pars I.). Zapiski Kavkazskago Muzeya, Seriya A (= Mémoires du Musée du Caucase, Série A) 6: 1-177 (in Russian, Latin title).

KIRITSHENKO A. N. 1938: Nastoyashchie poluzhestkokrylye nasekomye (Hemiptera) Nakhichevanskoy ASSR. [True bugs (Hemiptera) of Nakhichevan ASSR]. Trudy Zoologicheskogo Instituta Azerbaidzhanskiy Filial Akademiya Nauk SSSR 8: 75-121 (in Russian).

KIRITSHENKO A. N. 1957: Metody sbora nastoyashchikh poluzhestkokrylykh i izucheniya mestnykh faun. [Methods of collecting of true bugs and investigation of local faunas]. Izdatel'stvo Akademii Nauk SSSR, Moskva, Leningrad, 121 pp (in Russian).

KIRKALDY G. W. 1906: List of the genera of the pagiopodous Hemiptera-Heteroptera, with their type species, from 1758 to 1904 (and also of the aquatic and semi-aquatic Trochalopoda). Transactions of the American Entomological Society 32: 117-156b.

KIRKALDY G. W. 1908: Memoir on a new Heteropterous Hemiptera from Eastern Australia. Proceedings of the Linnean Society of New South Wales 32 [1907]: 768-788 + pl. 43.

KIRKALDY G. W. \& TORRE BUENO J. R. DE LA 1909: A catalogue of American aquatic and semi-aquatic Hemiptera. Proceedings of the Entomological Society of Washington 10 [1908]: 172-215.

KMENT P. \& BERAN L. 2011: Check-list of water bugs (Hemiptera: Heteroptera: Nepomorpha, Gerromorpha) in Croatia with two new records and three rediscoveries. Natura Croatica 20: 159-178.

KMENT P. \& JINDRA Z. 2008: Review of the family Gelastocoridae (Heteroptera: Nepomorpha) of south-eastern Asia. Pp. 189-213. In: GROZEVA S. \& SIMOV N. (eds): Advances in Heteroptera research. Festschrift in honour of 80th anniversary of Michail Josifov. PenSoft Publishers, Sofia, Moscow, 417 pp.
KMENT P., CARAPEZZA A., JINDRA Z. \& KONDOROSY E. 2017: Review of the genus Lanchnophorus (Hemiptera: Heteroptera: Rhyparochromidae) with description of three new species and other nomenclatural changes. Zootaxa 4226: 47-74.

KMENT P., HEMALA V. \& BAŇǍ̌ P. 2016: Rhyparoclava pyrrhocoroides, a new genus and species of autapomorphic Rhyparochromidae with clavate antennae from Madagascar (Hemiptera: Heteroptera). Acta Entomologica Musei Nationalis Pragae 56: 517-545.

KMENT P., HEMALA V. \& MALENOVSKÝ I. 2019: Scanning the Hyocephalidae: details of their external morphology with respect to phylogenetic relationships within Eutrichophora (Hemiptera: Heteroptera). Acta Entomologica Musei Nationalis Pragae 59: 423-441.

KOLENATI F. A. 1857: Meletemata Entomologica. Fasc. VI. Hemipterorum Heteropterorum Caucasi. Harpagocorisiae, monographice dispositae. Bulletin de la Société Impériale des Naturalistes de Moscou 29 [1856]: 419-491 + pl. III.

KORMILEV N. A. 1971: Ochteridae from the Oriental and Australian Regions (Hemiptera-Heteroptera). Pacific Insects 13: 429-444.

KORMILEV N. A. 1972: A new species of the genus Ochterus Latreille, 1807, from New Guinea (Hemiptera-Heteroptera: Ochteridae). Pacific Insects 14: 585-587.

KORMILEV N. A. 1973: Ochteridae from Western and Southern Africa (Hemiptera: Heteroptera). Occasional Papers of the California Academy of Sciences 106: 1-9.

KORMILEV N. A. \& DE CARLO J. A. 1952: Una especie nueva del genero 〈Ochterus〉 Latreille (1807) del Paraguay (Hemiptera, Ochteridae). Anales de la Sociedad Cientifica Argentina 154: 155-159.

KOVAC D. \& YANG C. M. 1990: A preliminary checklist of the semiaquatic and aquatic Hemiptera (Heteroptera: Gerromorpha and Nepomorpha) of Ulu Kinchin, Pahang, Malaysia. Malayan Nature Journal 43: 282-288.

KRÜGER A. \& DECKERT J. 2016: True bugs (Hemiptera-Heteroptera) of Botswana-Bibliographical inventory and new records. Zootaxa 4114(1): 33-63.

LAPORTE F. L. DE 1832-1833: Essai d'une classification systematique de l'ordre des Hémiptères. Magasin de Zoologie 2 (Suppl.): 1-88 [1832: 1-16, 1833: 17-88; Direction 63/1957].

LATREILLE P. A. 1804: Histoire naturelle, genérale et particuliere des crustacés et des insectes. Vol. 12. F. Dufart, Paris, 424 pp.

LATREILLE P. A. 1807: Genera crustaceorum et insectorum secundem ordinem naturalem in familias disposita iconibus exemplisque plurimis explicata. Tomus tertius. Amand Kœnig, Parisiis et Argentorati, $259 \mathrm{pp}$.

LATREILLE P. A. 1809: Genera crustaceorum et insectorum secundem ordinem naturalem in familias disposita iconibus exemplisque plurimis explicata. Tomus quartus et ultimus. Amand Kœnig, Parisiis et Argentorati, 599 pp +16 pls.

LATREILLE P. A. 1825: Familles naturelles du règne animal, exposées succintement et dans un ordre analytique avec l'indication de leurs genres. J. B. Baillière, Paris, 570 pp.

LEACH W. E. 1815: Entomology. Pp. 57-172. In: BREWSTER D. (ed.): The Edinburgh Encyclopcedia. Vol. 9. Blackwood, Edinburgh, 764 pp. + pls. CCLIII-CCLX.

LEE CH. E. \& KWON Y. J. 1991: Annotated check list of Hemiptera from Korea. Part I. Enicocephalomorpha, Nepomorpha, Leptopodomorpha \& Gerromorpha. Nature and Life 21: 1-10.

LEE CH. E., KWON Y. J. \& MIYAMOTO S. 1993: A checklist of Heteroptera from Chejudo. Nature and Life 23: 1-56.

LEE S., JEONG S., DUWAL R. K. \& SONG H. 2013: National list of species of Korea. Insect (Hemiptera I). National Institute of Biological Resources, Incheon, $238 \mathrm{pp}$.

LEONARD M. D. 1928: A list of the insects of New York. With a list of the spiders and certain other allied groups. Cornell University, Agricultural Experiment Station, Memoir 101: 1-1121.

Le PELETIER A. L. M. \& SERVILLE J. G. A. 1825: Tome dixième. [Vol. 10]. Pp. 1-344. In: OLIVIER G. A. (ed.): Encyclopédie méthodique. Agasse, Paris.

LI M., WANG J., TIAN X.-X., XIE Q., LIU H.-X. \& BU W.-J. 2012: Phylogeny of the true water bugs (Hemiptera-Heteroptera: Nepomorpha) based on four Hox genes. Entomotaxonomia 34: 35-44. 
LI T., HUA J.-M., WRIGHT A. M., CUI Y., XIE Q., BU W.-J. \& HILLIS D. M. 2014: Long-branch attraction and the phylogeny of true water bugs (Hemiptera: Nepomorpha) as estimated from mitochondrial genomes. BMC Evolutionary Biology 14(99): 1-12.

LI Z.-J., WU X.-P., CHEN X.-L. \& LIU C.-M. 2008: Report on scientific survey on Jiulingshan Reserve of Jiangxi. Science Press, Beijing, xi +313 pp. +18 plates (in Chinese).

LINDBERG H. 1922: Vezeichnis der von John Sahlberg und Uunio Saalas in den Mittelmeergebieten gesammelten semiaquatilen und aquatilen Heteropteren. Notulae Entomologicae 2: 15-19 + 46-49.

LINDBERG H. 1929: Inventa entomologica itineris Hispanici et Maroccani, quod a. 1926 fecerunt Harald et Håkan Lindberg. I. Hemiptera Heteroptera Hydrobiotica. Commentationes Biologicae 3(4): 1-12 +2 pls.

LINDBERG H. 1948: On the insect fauna of Cyprus. Results of the expedition of 1939 by Harald, Håkan and P. H. Lindberg. I-II. I. Introduction. II. Heteroptera und Homoptera Cicadina der Insel Zypern. Commentationes Biologicae 10(7): 1-175.

LINDBERG H. 1959: Hemiptera Insularum Caboverdensium. Commentationes Biologicae 19(1) [1958]: 1-246.

LINNAVUORI R. 1960: Hemiptera of Israel. I. Annales Zoologici Societatis Zoologica 'Vanamo'22(1): 1-71.

LINNAVUORI R. 1964: Hemiptera of Egypt, with remarks on some species of the adjacent Eremian region. Annales Zoologici Fennici 1: 306-356.

LINNAVUORI R. E. 1971: Hemiptera of the Sudan, with remarks on some species of the adjacent countries. 1. The aquatic and subaquatic families. Annales Zoologici Fennici 8: 340-366.

LINNAVUORI R. E. 1980: Hemiptera of the Sudan, with remarks on some species of the adjacent countries. 8. Additiones and correctiones. Biogeography. Acta Entomologica Fennica 36: 1-53.

LINNAVUORI R. E. 1981: Hemiptera of Nigeria, with remarks on some species of the adjacent countries. 1 . The aquatic and subaquatic families, Saldidae and Leptopodidae. Acta Entomologica Fennica 37: 1-39.

LINNAVUORI R. E. 1982: Some Hemipterous fauna from Somalia and Ethiopia. Monitore Zoologico Italiano 16(Suppl.): 1-33.

LINNAVUORI R. E. 1986: Heteroptera of Saudi Arabia. Pp. 31-197. In: BÜTTIKER W. \& KRUPP F. (eds): Fauna of Saudi Arabia. Vol. 8. Pro Entomologia c/o Natural History Museum, Basel, 461 pp.

LINNAVUORI R. E. 1989: Heteroptera of Yemen and South Yemen. Acta Entomologica Fennica 54: 1-40.

LINNAVUORI R. E. \& HOSSEINI R. 2000: Heteroptera of Guilan with remarks on species of the adjacent areas. Part 1. Guilan University Press, Rasht, [1999], 94 pp.

LINNAVUORI R. E., KMENT P. \& CARAPEZZA A. 2011: Order Hemiptera, suborder Heteroptera. Infraorders Nepomorpha, Gerromorpha, and Leptopodomorpha. Pp. 72-107. In: HARTEN A. VAN (ed.): Arthropod fauna of the United Arab Emirates. Vol. 4. Multiply Marketing Consultancy Services, Abu Dhabi, 816 pp.

LIU G.-Q. \& BU W.-J. 2009: Hemiptera: Heteroptera. The Fauna of Hebei, China. China Agricultural Science and Technology Press, 528 pp. + pls. I-VIII.

LLANO C. \& GUTIÉRREZ Y. 2015: Primer registro de la familia Ochteridae (Hemiptera: Heteroptera: Nepomorpha), con el género Ochterus Latreille, para la biota del departamento de Caldas. Boletín Cientifico Museo de Historia Natural Universidad de Caldas 19: 376-380.

L'MOHDI O., EL HAISSOUFI M., BENNAS N. \& MILLAN A. 2008: Contribution à la connaissance des Hétéroptères aquatiques du bassin versant Laou. Pp. 67-74. In: BAYED A. \& ATER M. (eds): Du bassin versant vers la mer: Analyse multidisciplinaire pour une gestion durable. Travaux de l'Institut Scientifique, Série Générale (Rabat) 5: i-iv + 1-122.

LÓPEZ RUF M., PÉREZ GOODWYN P. \& MARTINS-NETO R. G. 2005: New Heteroptera (Insecta) from the Santana Formation, Lower Cretaceous (Northeastern Brazil), with description of a new family and new taxa of Naucoridae and Gelastocoridae. Gaea 1: 68-74.

MAHNER M. 1993: Systema Cryptoceratorum Phylogeneticum (Insecta, Heteroptera). Zoologica (Stuttgart) 48: i-ix + 1-302.

MANCINI C. 1939a: Osservazioni sugli Ochterus del Museo Civico di Storia Naturale di Genova (hemipt.). Bollettino della Società Entomologica Italiana 67: 123-126.
MANCINI C. 1939b: Hemiptera. Pp. 292-314. In: Spedizione Zoologica del Marchese Saverio Patrizi nel Basso Giuba e nell'Oltreguiba. Giugno-Agosto 1934 XII. Annali del Museo Civico di Storia Naturale “Giacomo Doria” 58: 218-234.

MANCINI C. 1952: Miscellanea emitterologica italiana. I. Bollettino della Società Entomologica Italiana 82: 56-64.

MANCINI C. 1956: Emitteri dell'Abissinia raccolti dal M.se Saverio Patrizi e da altri. Fragmenta Entomologica 2: 65-96.

MANZANO M. DEL R., NIESER N. \& CAICEDO G. 1995. Lista preliminar de heterópteros acuáticos en la Isla de Gorgona y Llanura del Pacífico. Biblioteca José Jerónimo Triana 11: 47-72.

MATOCQ A., PLUOT-SIGWALT D. \& ÖZGEN I. 2014: Terrestrial Hemiptera (Heteroptera) collected in South-East Anatolia (Diyarbakır, Mardin and Elazığ provinces) (Turkey): second list. Munis Entomology and Zoology 9(2): 884-930.

MATSUMURA S. 1905: Die Wasser-Hemipteren Japans. Journal of the Sapporo Agricultural College 2(2): 53-66.

MATSUMURA S. 1915: Uebersicht der Wasser-Hemipteren von Japan und Formosa. Entomological Magazine 1(3): 103-119 + pl. III.

MAW H. E. L., FOOTTIT R. G., HAMILTON K. G. A. \& SCUDDER G. G. E. 2000: Checklist of the Hemiptera of Canada and Alaska. NRC Research Press, Ottawa, viii +220 pp.

MAXWELL-LEFROY H. 1909: XXV. The insect fauna of Tirhut. I. Rhynchota Heteroptera. Records of the Indian Museum (Calcutta) 3: 301-338.

MAYR G. L. 1868: Reise der Österreichischen Fregatte Novara um die Erde in den Jahren 1857, 1858, 1859 unter den Befehlen des Commodore B. von Wüllerstorf-Urbair. Zoologischer Theil. Vol. 2, 1 Ab., $B$, Hemiptera. Kaiserlichen Akademie der Wissenschaften, Wien, vi +204 pp +5 pls.

MAZZUCCONI S. A., LÓPEZ RUF M. \& BACHMANN A. O. 2009: Hemiptera - Heteroptera: Gerromorpha y Nepomorpha. Pp. 167-231. In: DOMÍNGUEZ E. \& FERNÁNDEZ H. R. (eds): Macroinvertebrados bentónicos sudamericanos. Sistemática y biología. Fundación Miguel Lillo, Tucumán, 656 pp.

MELO A. L. DE \& NIESER N. 2004: Faunistical notes on aquatic Heteroptera of Minas Gerais (Brazil): an annotated list of Gerromorpha and Nepomorpha collected near Januária, MG. Lundiana 5: 43-49.

MELO M. C. 2015: Ochteroidea. Pp. 341-346. In: ROIG-JUÑENT S., CLAPS L. E. \& MORRONE J. J. (eds): Biodiversidad de Artropódos Argentinos. Vol. 3. Universidad Nacional de Tucumán, Facultad de Ciencias, San Miguel de Tucumán, 546 pp.

MENKE A. S. 1979: Family Ochteridae. Pp. 124-125. In MENKE A. S. (ed.): The semiaquatic and aquatic Hemiptera of California (Heteroptera: Hemiptera). Bulletin of the California Insect Survey 21: $\mathrm{i}-\mathrm{xi}+1-166$

MIYAMOTO S. 1960: Aquatic and semiaquatic Heteroptera from Shansi Province, North China (Hemiptera). Mushi 33: 73-84 + pls. 7-9.

MIYAMOTO S. 1961: Comparative morphology of alimentary organs of Heteroptera, with the phylogenetic consideration. Sieboldia 2 [1959]: $198-259+$ pls. $20-49$.

MIYAMOTO S. 1964: Heteroptera collected by the Second Kyushu University Expedition to the Yaeyama Group, 1963 (excluding the Lygaeidae and Reduviidae). Reports of the Committee on Foreign Scientific Research, Kyushu University 2: 99-110.

MIYAMOTO S. \& LEE CH. E. 1966: Heteroptera of Quelpart Island (Chejudo). Sieboldia 3: 313-426.

MONTANDON A. L. 1897: Viaggio di Leonardo Fea in Birmania e regioni vicine. LXXV. Hemiptera Cryptocerata. Annali del Museo Civico di Storia Naturale di Genova, Serie 2 17: 365-377.

MONTANDON A. L. 1898: Hémiptères Hétéroptères nouveaux des collections du Muséum de Paris. Bulletin du Muséum d'Histoire Naturelle (Paris) 4: 72-75.

MONTANDON A. L. 1910: Quelques types d'Hemiptères de Guerin Meneville des Collections du Musée Zoologique de l'Université de Naples (Notes synonymiques et observations diverses). Annuario del Museo Zoologico della R. Università di Napoli (Nuova Serie) 10(3): 1-4.

MONTANDON A. L. 1914: II. Naucoridae, Nerthridae, Belostomatidae et Nepidae. Pp. 115-130. In: Voyage de Ch. Alluaud et R. Jeannel en Afrique orientale, 1911-1912. Résultats scientifiques. Insectes Hémiptères. II. A. Schulz, Paris, 333 pp +12 pls. 
MONTROUZIER P. 1864: Hémipteres Hétéropteres. Pp. 223-242. In: PERROUD B.-P. \& MONTROUZIER P.: Essai sur la faune entomologique de Kanala (Nouvelle-Calédonie) et description de quelques especes nouvelles ou peu connues. Annales de la Société de la Linnéene de Lyon, Séries 2 11: 46-257.

MOREIRA F. F. F., BARBOSA J. F., RIBEIRO J. R. I. \& ALECRIM V. P. 2011: Checklist and distribution of semiaquatic and aquatic Heteroptera (Gerromorpha and Nepomorpha) occurring in Brazil. Zootaxa 2958: 1-74.

MOREIRA F. F. F., RODRIGUES H. D. D., SITES R. W., CORDEIRO I. DA R. S. \& MAGALHÃES O. M. 2019: Order Hemiptera. Pp. 175-216. In: HAMADA N., THORP J. H. \& ROGERS D. C. (eds): Thorp and Covich's Freshwater Invertebrates (Fourth Edition). Vol. 3: Keys to Neotropical Hexapoda. Elsevier, $836 \mathrm{pp}$.

MUÑOZ RIVIAUX S., MOREIRA F. F. F. \& NARANJO LÓPEZ C. 2010a: Checklist, distribution, and habitat of the semiaquatic and aquatic bugs from Cuba (Hemiptera: Heteroptera: Dipsocoromorpha, Leptopodomorpha, Gerromorpha and Nepomorpha). Zootaxa 2562: 1-23.

MUÑOZ RIVIAUX S., NARANJO LÓPEZ C. \& MOREIRA F. F. F. 2010b: Nueva lista taxonómica de heterópteros acuáticos y semiacuáticos de Cuba (Insecta: Hemiptera). Cocuyo 18: 39-44.

NARANJO C., RIVIAUX S. M., MOREIRA F. F. F. \& COURT R. C 2010: Taxonomy and distribution of aquatic and semiaquatic Heteroptera (Insecta) from Cuba. Revista de Biologia Tropical 58: 897-907.

NIESER N. 1975: The water bugs (Heteroptera: Nepomorpha) of the Guyana region. Studies of the Fauna of Suriname and other Guyanas 16: $1-310+24$ pls.

NIESER N. 1982: De nederlandse water - en oppervlakte wantsen (Heteroptera: Nepomorpha en Gerromorpha). [The Dutch aquatic and semiaquatic bugs (Heteroptera: Nepomorpha and Gerromorpha)]. Wetenschappelijke Mededelingen van de Koninklijke Nederlandse Natuurhistorische Vereniging 155: 1-103.

NIESER N. \& ALKINS-KOO M. 1991: The water bugs of Trinidad \& Tobago. University of West Indies, Department of Zoology, Occasional Paper 9: i-iii + 1-127.

NIESER N., BAENA M., MARTINEZ-AVILES J. \& MILLAN A. 1994: Claves para la identificación de los heterópteros acuáticos (nepomorpha \& gerromorpha [sic!]) de la Península Ibérica - Con notas sobre las especias de las Islas Azores, Baleares, Canarias y Madeira. In: PRAT N. (ed.): Claves de identificacion de la flora y fauna de las aquas continentales de la Peninsula Iberica, Publicacion No. 5. Asociacion Española de Limnologia, Madrid, 112 pp.

NIESER N. \& CHEN P.-P. 1992: Notes on Gelastocoridae and Ochteridae (Heteroptera) with the description of five new species. Storkia 1: 2-13.

NIESER N. \& CHEN P.-P. 1999: Sixteen new species of Nepomorpha mainly from Sulawesi. Notes on Malaysian aquatic and semiaquatic bugs (Heteroptera), VIII. Tijdschrift voor Entomologie 142: 77-123.

NIESER N., CHEN P.-P. \& CASPERS M. 2020: Taxonomic notes on toad bug genus Nerthra (Hemiptera: Heteroptera: Gelastocoridae) with description of a new species from Bonaire and Curaçao. Acta Entomologica Musei Nationalis Pragae 60(1): in press.

NIESER N. \& MELO A. L. DE 1997: Os heterópteros aquáticos de Minas Gerais. Editora UFMG, Belo Horizonte, 177 pp.

NIESER N. \& MONTES C. 1984: Lista faunistica y bibliografica de los Heteropteros acuaticos (Nepomorpha \& Gerromorpha) de España y Portugal. Asociacion Española de Limnologia, Madrid, 69 pp.

NIESER N. \& MOUBAYED Z. 1985: Les Hétéropteres aquatiques du Liban. I. Inventaire faunistique. Annales de Limnologie 21: 247-252.

OSHANIN B. 1909: Verzeichnis der Palaearktischen Hemipteren mit besonderer Berücksichtigung ihrer Verteilung im Russischen Reiche. I. Band. Heteroptera. III. Lieferung. Ezhegodnik Zoologicheskago Muzeya Imperatorskoy Akademii Nauk 14 (Prilozhenie): 587-1087 $\mathrm{pp}+4 \mathrm{pp}$ [index].

OSHANIN B. 1912: Katalog der paläarktischen Hemipteren (Heteroptera, Homoptera-Auchenorrhyncha und Psylloidea). R. Friedländer \& Sohn, Berlin, xiv +187 pp.

ÖZGEN İ., TOPDEMIR A. \& TANYILDIZI M. Ş. 2017: Some ecological and faunistical notes on insect fauna in Şahaplı stream (Turkey: Elazı Baskil). Munis Entomology and Zoology 12: 362-364.

PACKAUSKAS R. J. 2010: Catalog of the Coreidae, of leaf-footed bugs, of the New World. Fort Hays Studies, Fourth Series 5: 1-168.
PADILLA-GIL D. N. 2015: Gerromorpha y Nepomorpha (Heteroptera) del Pacífico de Colombia: lista de especies, distribución geográfica y altitudinal. Biota Colombiana 16(1): 20-35.

PAGOLA-CARTE S. \& RIBES J. 2007: Registros interesantes de heterópteros (Hemiptera: Heteroptera) en el País Vasco (norte de la Península Ibérica). Heteropterus Revista de Entomología 7: 25-31.

PAIVA C. A. 1919a: Rhynchota from the Garo Hills, Assam. Records of the Indian Museum (Calcutta) 16: 349-377 + pls. XXXIV-XXXVI.

PAIVA C. A. 1919b: VII. Aquatic and semi-aquatic Rhynchota from the Satara and Poona Districts. Pp. 152-156 + pl. III. In: ANNANDALE N. (ed.): The fauna of certain small streams in the Bombay Presidency. Records of the Indian Museum (Calcutta) 16: 109-161 + 7 pls.

PELLI A., NIESER N. \& MELO A. L. DE 2006: Nepomorpha and Gerromorpha (Insecta: Heteroptera) from the Serra da Canastra, southwestern Minas Gerais state, Brazil. Lundiana 7: 67-72.

PEREIRA D. L. V. \& MELO A. L. 2007: Aquatic and semiaquatic Heteroptera (Insecta) from Pitinga, Amazonas, Brazil. Acta Amazonica 37: 643-648.

POISSON R. A. 1925: Quelques Hémipteres aquatiques des environs de Banyuls (Pyrénées-Orientales). Bulletin de la Société Entomologique de France 1925: 270-273.

POISSON R. A. 1934: Contribution a l'étude de la faune du Mozambique. Voyage de M. P. Lesne (1928-1929) (15e note). Hémipteres aquatiques. Bulletin de la Société Zoologique de France 59: 87-103.

POISSON R. A. 1949: Hémiptères aquatiques. Parc National Albert, I. Mission G. F. de Witte (1933-1935) 58: 3-94.

POISSON R. A. 1954: XXX. Hydrocorises (2e contribution) (Mission Lamotte et Roy, juillet-décembre 1951). In: La Réserve Naturelle Intégrale du Mt Nimba. Fascicule II. Mémoires de l'Institut Francais d'Afrique Noire 40: 359-370.

POISSON R. A. 1957: Hétéroptères aquatiques. Faune de France. Vol. 61. Fédération Française des Sociétés de Sciences Naturelles, Paris, $263 \mathrm{pp}$.

POISSON R. A. 1968a: Contributions a la connaissance de la faune entomologique de la Cote d'Ivoire (J. Decelle 1961-1964). Annales de Musée Royal de l'Afrique Centrale, Serie in- $8^{\circ}$, Sciences Zoologiques 165: 399-403.

POISSON R. A. 1968b: Aquatic Heteroptera. In: SYMOENS J.-J. (ed.): Hydrobiological survey of the Lake Bangweulu, Luapula River Basin. Volume XIV, fascicule 11. Cercle Hydrobiologique de Bruxelles, Bruxelles, $51 \mathrm{pp}$.

POISSON R. A. \& SALLIER DUPIN F. DE 1969: Aquatic Heteroptera from the Luanza Drainage Area. In: SYMOENS J.-J. (ed.): Hydrobiological survey of the Lake Bangweulu, Luapula River Basin. Volume $X I V$, fascicule 12. Cercle Hydrobiologique de Bruxelles, Bruxelles, $26 \mathrm{pp}+2$ pls.

POLHEMUS D. A. 1992a: The first records of the families Ochteridae and Hebridae (Heteroptera) from the granitic Seychelles, with descriptions of two new species. Journal of the New York Entomological Society 100: 418-423.

POLHEMUS D. A. \& POLHEMUS J. T. 1988a: Family Ochteridae Kirkaldy, 1906. The Velvety Shore Bugs. Pp. 541-543. In: HENRY T. J. \& FROESCHNER R. C. (eds): Catalog of the Heteroptera, or True Bugs, of Canada and the Continental United States. E. J. Brill, Leiden, New York, København, Köln, xix + 958 pp.

POLHEMUS D. A. \& POLHEMUS J. T. 1988b: Family Gelastocoridae Kirkaldy, 1897. The Toad Bugs. Pp. 136-139. In: HENRY T. J. \& FROESCHNER R. C. (eds): Catalog of the Heteroptera, or True Bugs, of Canada and the Continental United States. E. J. Brill, Leiden, New York, København, Köln, xix + 958 pp.

POLHEMUS D. A. \& POLHEMUS J. T. 1998: A biodiversity survey of aquatic insects in the Ajkwa River Basin and adjacent areas, Irian Jaya, Indonesia. Tropical Biodiversity 5(3): 197-216.

POLHEMUS D. A. \& POLHEMUS J. T. 2008: A new Indian Ocean species of Ochterus from the island of Mauritius (Hemiptera: Heteroptera: Ochteridae). Acta Entomologica Musei Nationalis Pragae 48: 281-288.

POLHEMUS D. A. \& POLHEMUS J. T. 2012: Guide to the aquatic Heteroptera of Singapore and Peninsular Malaysia. IX. Infraorder Nepomorpha, families Ochteridae and Gelastocoridae. Raffles Bulletin of Zoology 60: 343-359. 
POLHEMUS D. A. \& POLHEMUS J. T. 2013: Aquatic and semi-aquatic Heteroptera. Pp. 67-100. In: GERLACH J. (ed.): Odonata, Hemiptera, Hymenoptera and other insects of the Seychelles islands. Siri Scientific Press, Manchester, 396 pp. +4 pls.

POLHEMUS D. A. \& POLHEMUS J. T. 2014: Ocyochterus irmae, a beautiful new species of Ochteridae (Heteroptera) from the Northern Andes. Tijdschrift voor Entomologie 157: 163-171.

POLHEMUS D. A. \& POLHEMUS J. T. 2015: Ochteridae. Pp. 205-212. In: TRAN A. D. (ed.): Water bugs of Singapore and Peninsular Malaysia. Lee Kong Chian Natural History Museum, Singapore, 334 pp.

POLHEMUS D. A. \& POLHEMUS J. T. 2016: Revision of the genus Ochterus (Hemiptera: Heteroptera: Ochteridae) in Mesoamerica and the United States. Tijdschrift voor Entomologie 159: 9-75.

POLHEMUS J. T. 1973: Notes on aquatic and semiaquatic Hemiptera from the Southwestern United States (Insecta: Hemiptera). Great Basin Naturalist 33: 113-119.

POLHEMUS J. T. 1976: Chapter 9: Shore bugs (Hemiptera: Saldidae, etc.). Pp. 225-262. In: CHENG L. (ed.): Marine insects. NorthHolland Publishing Company, Amsterdam, Oxford, xii +581 pp.

POLHEMUS J. T. 1979: Results of the Austrian-Ceylonese Hydrobiological mission 1970, of the Institute of Zoology of the University of Vienna (Austria) and the Department of Zoology of the University of Sri Lanka, Vidyalankara Campus, Kelaniya. Part XIX: Aquatic and semiaquatic Hemiptera of Sri Lanka from the Austrian Indo-Pacific Expedition, 1970-71. Bulletin of the Fisheries Research Station Sri Lanka 29: 89-113.

POLHEMUS J. T. 1992b: Nomenclatural notes on aquatic and semiaquatic Heteroptera. Journal of the Kansas Entomological Society 64(4) [1991]: 438-443.

POLHEMUS J. T. 1995a: Family Ochetridae Kirkaldy, 1906 - velvety shore bugs. Pp. 25-26. In: AUKEMA B. \& RIEGER CH. (eds): Catalogue of the Heteroptera of the Palaearctic Region. Vol. 1, Enicocephalomorpha, Dipsocoromorpha, Nepomorpha, Gerromorpha and Leptopodomorpha. The Netherlands Entomological Society, Amsterdam, xxvi $+222 \mathrm{pp}$.

POLHEMUS J. T. 1995b: Family Gelastocoridae Kirkaldy, 1897 - toad bugs. Pp. 23-25. In: AUKEMA B. \& RIEGER CH. (eds): Catalogue of the Heteroptera of the Palaearctic Region. Vol. 1, Enicocephalomorpha, Dipsocoromorpha, Nepomorpha, Gerromorpha and Leptopodomorpha. The Netherlands Entomological Society, Amsterdam, $\mathrm{xxvi}+222 \mathrm{pp}$.

POLHEMUS J. T. 1997: New state and U.S. records and other distributional notes for Heteroptera (Insecta). Entomological News 108: 305-310.

POLHEMUS J. T. \& POLHEMUS M. S. 1976: Aquatic and semiaquatic Heteroptera of the Grand Canyon (Insecta: Hemiptera). Great Basin Naturalist 36: 221-226.

POPOV YU. A. 1971: Istoricheskoe razvitie poluzhestkokrylykh infraotryada Nepomorpha (Heteroptera). [Historical development of true bugs of the infraorder Nepomorpha (Heteroptera)]. Trudy Paleontologicheskogo Instituta AN SSSR 129: 1-230 + pls 1-9 (in Russian).

POPOV Yu. A 1986: Podotryad Cimicina (= Heteroptera). Pp. 54-83. In: Nasekomye v rannemelovykh ekosistemakh Zapadnoy Mongolii. Somestnaya Sovetsko-Mongol'skaya Paleontologicheskaya Ekspeditsiya. [Insects in Early Cretaceous ecosystems of Western Mongolia. The Joint Soviet-Mongolian Palaeontological Expedition.] Trudy. [Transactions]. Vol. 28. Nauka, Moskva, 214 pp (in Russian).

POPOV Yu. A., DOLLING W. R. \& WHALLEY P. E. S. 1994: British Upper Triassic and Lower Jurassic Heteroptera and Coleorrhyncha (Insecta: Hemiptera). Genus 5: 307-347.

POPOV Yu. A. \& HEISS E. 2014a: Riegerochterus baehri gen. nov. and spec. nov., the first fossil velvety bug (Hemiptera: Heteroptera, Ochteridae) from Dominican Amber. Andrias 20: 185-190.

POPOV YU. A. \& HEISS E. 2014b: Grimaldinia pronotalis n. gen., n. sp. from Mid-Cretaceous Burmese Amber (Hemiptera: Heteroptera Leptopodidae, Leptosaldinae). Zootaxa 3878(5): 444-450.

PRIESNER H. \& ALFIERI A. 1953: A review of the Hemiptera Heteroptera known to us from Egypt. Bulletin de la Société Fouad I ${ }^{\text {er }}$ d'Entomologie 37: 1-119.

PROTIĆ LJ. 1998: Catalogue of the Heteroptera fauna of Yugoslav countries. Part one. Prirodnjački Muzej u Beogradu, Posebna Izdanja 38: 1-215.
PUTON A. 1869: Catalogue des Hémiptères Hétéroptères d'Europe. Deyrolle, Paris, vii +40 pp.

PUTON A. 1880: Synopsis des Hémiptères-Hétéroptères de France. $3 e$ partie. Reduvides, Saldides, Hydrocorises. Danel, Lille, pp. 161-243. [Preprint of Mémoires de la Société des Sciences de Lille, Séries 4 9 [1881]: 65-150.]

RÉDEI D. 2017: A revision of Sagriva (Hemiptera: Heteroptera: Dinidoridae). Acta Entomologica Musei Nationalis Pragae 57: 73-95.

REUTER O. M. 1888: Revisio synonymica Heteropterorum Palaearcticorum quae descripserunt auctores vetustiores (Linnaeus 1758 - Latreille 1806). Finnische Literatur-Gesellschaft, Helsingfors, 485 pp [I: pp. 1-73, II: pp. 74-485]. [Also published in Acta Societatis Scientiarum Fennicae 15 [1888]: 241-315 [part I], 443-812 [part II].

REUTER O. M. 1910: Neue Beiträge zur Phylogenie und Systematik der Miriden nebst einleitenden Bemerkungen über die Phylogenie der Heteropteren-Familien. Acta Societatis Scientiarum Fennica 37(3): $1-172+1 \mathrm{pl}$.

RIEGER CH. 1976: Skelett und Muskulatur des Kopfes und Prothorax von Ochterus marginatus Latreille. Zoomorphologie 83: 109-191.

RIEGER CH. 1977: Neue Ochteridae aus der Alten Welt. Deutsche Entomologische Zeitschrift, Neue Folge 24: 213-217.

ROBACK S. S. 1958: New records of aquatic Heteroptera from the United States and Canada. Transactions of the American Entomological Society 84: 1-11.

SCHELL D. V. 1943a: The Ochteridae (Hemiptera) of the Western Hemisphere. Journal of the Kansas Entomological Society 16(1): 29-36. [January 1943]

SCHELL D. V. 1943b: The Ochteridae (Hemiptera) of the Western Hemisphere. Journal of the Kansas Entomological Society 16(2): 37-47. [April 1943]

SCHOUTEDEN H. 1907: Les types d'Hémiptères de Montrouzier. Annales de la Société Entomologique de Belgique 51: 106-118.

SCHOUTEDEN H. 1933: Resultats Scientifiques du Voyage aux Indes Orientales Néerlandaises de LL. AA. RR, le Prince et la Princesse Léopold de Belgique. Hemiptera-Heteroptera. Mémoires du Musée Royal d'Histoire Naturelle de Belgique 4(8): 43-70.

SCHUH R. T. \& SLATER J. A. 1995: True bugs of the world (Hemiptera: Heteroptera). Classification and natural history. Comstock Publishing Associates, Cornell University Press, Ithaca \& London, xii $+336 \mathrm{pp}$.

SCHUH R. T. \& WEIRAUCH CH. 2020: True bugs of the World (Hemiptera: Heteroptera). Classification and Natural history. Second edition. Monograph Series. Vol. 8. Siri Scientific Press, Manchester, $767 \mathrm{pp}+32$ pls.

SCOTT J. 1874: On a collection of Hemiptera Heteroptera from Japan. Descriptions of various new genera and species. Annals and Magazine of Natural History, Series 4 14: 426-452.

SEABRAA. F. DE 1926: Revisão dos Hemípteros Heterópteros da fauna paleárctica existens no Museu Zológico da Universidade de Coimbra. Memórias e Estudos do Museu Zoológico da Universidade de Coimbra, Serie 1 10: 1-234.

SERVADEI A. 1967: Rhynchota (Heteroptera, Homoptera Auchenorrhyncha). Catalogo topografico e sinonimico. Fauna d'Italia. Calderini, Bologna, $851 \mathrm{pp}$.

SHCHERBAKOV D. E. \& POPOV Yu. A. 2002: 2.2.1.2.5. Superorder Cimicidea Laicharting, 1781. Order Hemiptera Linné, 1758. The Bugs, Cicadas, Plantlice, Scale Insects, etc. (= Cimicida Laicharting, 1781, = Homoptera Leach, 1815 + Heteroptera Latreille, 1810). Pp. 143-157. In: RASNITSYN A. P. \& QUICKE D. L. J. (eds): History of insects. Kluwer Academic Publishers, New York, Boston, Doderecht, London, Moscow, xii +517 pp.

SHEN X.-CH., REN Y.-D., NIU Y., YIN X.-M., LIU X.-T., LIU X.-G., SUN H. \& MA X.-J. 2014: Insect fauna of Henan. Cataloque [sic!] and distribution. Science Press, Beijing, xxvi +1271 pp. + LIV pls.

SIENKIEWICZ I. 1964: The catalogue of the „, A. L. Montandon collection " of Palaearctic Heteroptera preserved in the "Grigore Antipa " Museum of Natural History, Bucharest. Grigore Antipa Museum of Natural History, Bucharest, 146 pp.

SINGH-PRUTHI H. 1925: The morphology of the male genitalia in Rhynchota. Transactions of the Entomological Society of London 1925: $127-267+32$ pls.

SLIMANI N., MOULET P., CHEN P.-P., NIESER N., PLUOT-SIGWALT 
D., BOUMAÏZA M. \& GUILBERT E. 2015: Checklist, distribution, and a new record of Nepomorphan water bugs (Hemiptera: Heteroptera) in northern Tunisia. Zootaxa 3981(2): 151-176.

SMITH J. B. 1890: Catalogue of Insects found in New Jersey. Pp. 1-486. In: Final Report of the State Geologist. Vol. II. Mineralogy, Botany, Zoölogy. Part 2. Zoölogy. Geological Survey of New Jersey, Trenton.

SNOW F. H. 1906b: Some results of the University of Kansas entomological expeditions to Arizona in 1904 and 1905. Transactions of the Kansas Academy of Science 20: 155-181.

SOUZA M. A. A. DE, MELO A. L. DE \& VIANNA G. J. C. 2006: Heterópteros aquáticos oriundos do município de Mariana, MG. Neotropical Entomology 35: 803-810.

STÅL C. 1855: Hemiptera från Kafferlandet. Öfversigt af Kongliga Vetenskaps-Akademiens Forhandlingar 12: 27-47.

STÅL C. 1866: Hemiptera Africana. Vol. III. Officina Norstedtiana, Holmiae, [1865], $200 \mathrm{pp.}$

STÅL C. 1876: Enumeratio Hemipterorum. Bidrag till en förteckning öfver alla hittills kända Hemiptera, jemte systematiska meddelanden. 5. Kongliga Svenska Vetenskaps-Akademiens Handlingar 14(4): $1-162$.

STEVENS L. E. \& POLHEMUS J. T. 2008: Biogeography of aquatic and semiaquatic Heteroptera in the Grand Canyon ecoregion, southeastern USA. Monographs of the Western North American Naturalist 4: 38-76.

STOIANOVA D. \& SIMOV N. 2016: New records of aquatic true bugs (Hemiptera: Heteroptera: Nepomorpha) from Bulgaria. Acta Zoologica Bulgarica 68: 497-502

TAKAHASHI R. 1921: [Observations on the Ochteridae (Rhynchota).] Transactions of the Natural History Society of Formosa 11: 119-125 (in Japanese).

TAKAHASHI R. 1923: Observations on the Ochteridae. Bulletin of the Brooklyn Entomological Society 18: 67-68.

TAKENO K. 1998: Enumeratio of the Heteroptera in Mt. Hikosan, Western Japan with their hosts and prays I. Esakia 38: 29-53.

TAMANINI L. 1979: Eterotteri acquatici (Heteroptera: Gerromorpha, Nepomorpha). Guide per il riconoscimento delle specie animali delle acque interne italiane. Vol. 6. Consiglio Nazionale delle Ricerche, Verona, $106 \mathrm{pp}$.

TAMANINI L. 1981: Gli eterotteri della Basilicata e della Calabria (Italia meridionale) (Hemiptera Heteroptera). Memorie del Museo Civico di Storia Naturale di Verona (IIa Serie) Sezione Scienze della Vita (A: Biologica) 3: 1-145.

THIRUMALAI G. 1989: Aquatic and semi aquatic Hemiptera (Insecta) of Javadi hills, Tamil Nadu. Records of the Zoological Survey of India Occasional Paper 118: $1-63+3$ pls.

THIRUMALAI G. 1989: Aquatic and semi aquatic Hemiptera (Insecta) of Javadi hills, Tamil Nadu. Records of the Zoological Survey of India, Occasional Paper 118: $1-63+3$ pls.

THIRUMALAI G. 1999: A checklist of aquatic and semi-aquatic Hemiptera (Insecta) of Tamil Nadu. Zoos' Print Journal 14: 132-135.

THIRUMALAI G. 2001: Insecta: Aquatic and semi-aquatic Heteroptera. Pp. 111-127. In: Fauna of Nilgiri Biosphere Reserve. Conservation Area Series. Vol. 11. Zoological Survey of India, Kolkata, 330 pp.

THIRUMALAI G. 2007: A synoptic list of Nepomorpha (Hemiptera: Heteroptera) from India. Records of the Zoological Survey of India, Occasional Paper 273: 1-84.

THIRUMALAI G. \& SHARMA R. M. 2002: Aquatic and semiaquatic Heteroptera (Insecta). Pp. 105-116. In: Fauna of Ujani (Maharashtra). Wetland Ecosystem Series 3. Zoological Survey of India, Kolkata, 196 pp.

THIRUMALAI G. \& SHARMA R. M. 2012: Insecta: Hemiptera (aquatic and semi-aquatic). Pp. 467-472. In: Fauna of Maharashtra. State Fauna Series. Vol. 20 (Part 2). Zoological Survey of India, Kolkata, $673 \mathrm{pp}$.

THIRUMALAI G., SHARMA R. M. \& CHANDRA K. 2007: A checklist of aquatic and semiaquatic Hemiptera (Insecta) of Madhya Pradesh. Records of the Zoological Survey of India 107(4): 71-91.

TINERELLA P. P., TAYLOR S. J. \& MCPHERSON J. E. 2009: New records and a checklist of aquatic true bugs (Heteroptera: Nepomorpha) from Illinois. Journal of the Kansas Entomological Society $\mathbf{8 2}$ 293-299.
TODD E. L. 1955: A taxonomic revision of the family Gelastocoridae (Hemiptera). University of Kansas Science Bulletin 37: 277-475.

TODD E. L. 1961: A checklist of the Gelastocoridae (Hemiptera). Proceedings of the Hawaiian Entomological Society 17: 461-476.

TOMOKUNI M. 1981: Heteroptera of the Izu Peninsula, Central Japan. Memoirs of the National Science Museum (Tokyo) 14: 104-116 (in Japanese, English summary).

TORRE BUENO J. R. DE LA 1905: A list of certain families of Hemiptera occurring within seventy miles of New York. Journal of the New York Entomological Society 13: 29-47.

TORRE BUENO J. R. DE LA 1906: The cryptocerate Hemiptera of America in the writings of professor Arnold L. Montandon. Proceedings of the Entomological Society of Washington 8: 45-58.

TORRE BUENO J. R. DE LA 1908: Hemiptera Heteroptera of Westchester County, N. Y. Journal of the New York Entomological Society 15: 223-238.

TORRE BUENO J. R. DE LA \& BRIMLEY C. S. 1907: On some Heteropterous Hemiptera from N. Carolina. Entomological News 18: $433-443$

TSAI J.-F., RÉDEI D., YEH G.-F. \& YANG M.-M. 2011: Jewel bugs of Taiwan (Heteroptera: Scutelleridae). National Chung Hsing University, Taichung, 309 pp.

UHLER P. R. 1876: List of the Hemiptera of the region West of the Mississippi river, including those collected during the Hayden explorations of 1873. Bulletin of the Geological and Geographical Survey of the Territories 1: 269-361 + pls. 19-21.

UHLER P. R. 1893: A list of the Hemiptera-Heteroptera collected in the Island of St. Vincent by Mr. Herbert H. Smith; with descriptions of new genera and species. Proceedings of the Zoological Society of London 1893: 705-719.

UHLER P. R. 1894: On the Hemiptera-Heteroptera of the Island of Grenada, West Indies. Proceedings of the Zoological Society of London 1894: $167-224$.

VAN DUZEE E. P. 1917: Catalogue of the Hemiptera of America North of Mexico excepting the Aphididae, Coccididae and Aleurodidae. In: WOODWORTH C. W. (ed.): University of California Publications in Entomology, Vol. 2. University of California Press, Berkeley, xiv $+902 \mathrm{pp}$.

VAN DUZEE E. P. 1923: Expedition of the California Academy of Sciences to the Gulf of California in 1921. The Hemiptera (true bugs, etc.). Proceedings of the California Academy of Sciences, Fourth Series 12: 123-200.

VIANNA G. J. C. \& MELO A. L. DE 2003: Distribution patterns of aquatic and semi aquatic Heteroptera in Retiro das Pedras, Brumadinho, Minas Gerais, Brazil. Lundiana 4: 125-128.

VIDAL J. P. 1937: Contribution à l'étude des Hémiptères-Hétéroptères du Maroc. Bulletin de la Société d'Histoire Naturelle de l'Afrique du Nord 28: 185-208+2 pls.

VINOKUROV N. N., KANYUKOVA E. V. \& GOLUB V. B. 2010: Catalogue of the Heteroptera of the Asian part of Russia. Nauka, Novosibirsk, 323 pp.

WANG, Y. H., CUI Y., RÉDEI D., BAŇAř P., XIE Q., ŠTYS P., DAMGAARD J., CHEN P. P., YI W. B., WANG Y., DANG K., LI C. R. \& BU W. J. 2016: Phylogenetic divergences of the true bugs (Insecta: Hemiptera: Heteroptera), with emphasis on the aquatic lineages: the last piece of the aquatic insect jigsaw originated in the Late Permian/ Early Triassic. Cladistics 32: 390-405.

WEIRAUCH CH., SCHUH R. T., CASSIS G. \& WHEELER W. C. 2019: Revisiting habitat and lifestyle transitions in Heteroptera (Insecta: Hemiptera): insights from a combined morphological and molecular phylogeny. Cladistics 35: 67-105.

WHALLEY P. E. S. 1985: The systematics and palaeogeography of the Lower Jurassic insects of Dorset, England. Bulletin of the British Museum of Natural History, Geology Series_39: 107-189.

WILSON C. A. 1958: Aquatic and semiaquatic Hemiptera of Mississippi. Tulane Studies in Zoology 6: 115-170.

WIRTNER P. M. 1904: A preliminary list of the Hemiptera of Western Pennsylvania. Annals of the Carnegie Museum 3: 183-232.

WU Cн.-F. F. 1935: Catalogus Insectorum Sinensium. Volume II. The Fan Memorial Institute of Biology, Peiping, 634 pp. 
XIE T.-Y. \& LIU G.-Q. 2013: Catalogue of Nepomorpha (Hemiptera: Heteroptera) from China (I). Sciencepaper Online, 18 pp (in Chinese, English summary) [available on: http://www.paper.edu.cn/releasepaper/content/201305-98]

YANO K., MIYAMOTO S. \& GABRIEL B. P. 1981: Faunal and biological studies on the insects of paddy fields in Asia. IV. Aquatic and semiaquatic Heteroptera from the Philippines. Esakia 16: 5-32.

YANO S., KIKUHARA Y., TAKECHI L. \& WATANABE K. 2012: List of species of Heteroptera (Insecta: Hemiptera) in Matsuyama City, Ehime Prefecture, Shikoku, Japan. 2nd version. Pp. 81-100. In: ISHIKAWA K. (ed.): Checklist of the wild animals, fungi, and plants of Matsuyama City, 2012. Department of Environment, Matsuyama, 404 pp (in Japanese, English abstract).

YAO Y.-ZH., CAI W.-ZH. \& REN D. 2007: Pristinochterus gen. n. (Hemiptera: Ochteridae) from the Upper Mesozoic of northeastern China. European Journal of Entomology 104: 827-835.

YAO Y.-ZH., REN D., SHIH CH.-K. \& ZHANG W.-T. 2010: Chapter 15. Heteroptera - smelly defense or piercing offense. Pp. 139-157. In: DONG R., SHIH CH.-K., GAO T.-P., YAO Y.-Zн. \& ZHAO Y.-Y. (eds): Silent stories - Insect fossil treasures from Dinosaur Era of the Northeastern China. Science Press, Beijing, 322 pp.
YAO Y.-ZH., ZHANG W.-T., REN D. \& SHIH CH.-K. 2011: New fossil Ochteridae (Hemiptera: Heteroptera: Ochteroidea) from the Upper Mesozoic of north-eastern China, with phylogeny of the family. Systematic Entomology 36: 589-600.

YE ZH., DAMGAARD J., YANG H.-H., HEBSGAARD M. B., WEIR T. \& BU W.-J. 2020: Phylogeny and diversification of the true water bugs (Insecta: Hemiptera: Heteroptera: Nepomorpha). Cladistics 36: 72-87.

ZETTEL H. \& LANE D. J. W. 2010: A new species of Ochterus Latreille (Heteroptera: Ochteridae) from Brunei. Zeitschrift der Arbeitsgemeinschaft Österreichischer Entomologen 62: 97-101.

ZHANG SH.-M. (ed.) 1994: Cataogus [sic!] of insects from survey Jtangxi [sic!], China. Jiangxi Science and Technology Press, Nanchang, v + 273 pp (in Chinese, English title).

ZIMMERMANN G. 1982: Beiträge zur Wasserwanzen-Fauna Griechenlands I (Heteroptera, Nepomorpha, Gerromorpha, Saldidae). Marburger Entomologische Publikationen 1(7): 183-216.

ZRZAVÝ J. 1990: Evolution in antennal sclerites in Heteroptera (Insecta). Acta Universitatis Carolinae Biologica 34: 189-227. 